Eidg. Technische Hochschule, Zürich - Fortbildungskurs der EAWAG, r964

\title{
Hauptwirkungen und Wechselwirkungen einiger Betriebsparameter im Belebtschlammsystem; Ergebnisse mehrjähriger Grossversuche
}

Von K.WuHRMANN, EAwag, Zürich 


\section{Einleitung}

Eine Belebtschlammanlage ist ein kontinuierliches Fermentationssystem, dessen Arbeitsziel der vollständige Verbrauch der zufliessenden Fermentationssubstrate (d.h. der organischen Verunreinigungsstoffe des Abwassers) darstellt. Bei ihrem Betrieb sind hydraulische Raumbelastung $\left(R_{h}, \mathrm{~m}^{3}\right.$ Abwasser $/ \mathrm{m}^{3}$ Belüftungsraum und Tag, gleichbedeutend mit dem reziproken Wert der Belüftungszeit $\mathrm{I} / t$ ), die Schlammkonzentration im Belüftungsbecken $x_{1}^{\prime}$ und die Sauerstoffspannung im Belüftungsbecken $O$ innerhalb gewisser Grenzen frei wählbar. Die Temperatur $t^{\circ}$ im Belüftungsbecken, die einen unmittelbaren Einfluss auf die Geschwindigkeit aller biochemischen Reaktionen ausübt, muss hingenommen werden. Desgleichen ist die Konzentration $s_{R}$ und Zusammensetzung des Abwassers (d.h. die Qualität des Milieus als Nährboden für die Mikroorganismen des Belebtschlamms) meistens eine unbeeinflussbare Grösse.

Die Abhängigkeit der Reinigungswirkung einer Belebtschlammanlage von den manipulierbaren Betriebsparametern wird in der Abwasserliteratur mit Hilfe verschiedenartigster, empirischer Korrelationen darzustellen versucht, um zu Beziehungen zu gelangen, welche u.a. dem Ingenieur für Dimensionierungsberechnungen dienen könnten. Begriffe wie Reinigungseffekt, Abbauleistung, Schlammbelastung, Schlammalter usw. und Beziehungen zwischen solchen Grössen (Beispiele: Abhängigkeit des Reinigungseffektes von der Schlammbelastung oder von einem "Belastungsparameter" nach FAIR und THOMAS [4], Abhängigkeit der Úberschuss- 
schlammbildung vom Schlammalter oder von der Schlammbelastung usw.), erscheinen aber nur dann sinnvoll, wenn sie auch theoretisch begründet sind. Im Interesse der experimentellen Ökonomie bei der Planung, Durchführung und Auswertung des nachfolgend beschriebenen «Faktorenversuches" erachteten wir es deshalb als zweckmässig, uns von der Theorie der kontinuierlichen Fermentationen, wie sie auf Grund der Arbeiten von Monod [9], Novick und SzILARD [Io] und HerberT et al. [6] entwickelt wurde, leiten zu lassen. In der deutschsprachigen Abwasserliteratur ist diesen Zusammenhängen bisher leider wenig Aufmerksamkeit geschenkt worden, so dass wir einleitend kurz darauf eintreten müssen, weil wir sie bei der späteren Darstellung benützen werden.

\section{Theoretische Grundlagen}

\subsection{Organismenwacbstum und Substratperbrauch}

Das Wachstum von Mikroorganismen in einer physiologisch vollwertigen Nährlösung verläuft streng gesetzmässig, und die Abhängigkeit der Wachstumsgeschwindigkeit von der Konzentration einer einzigen, geschwindigkeitsbegrenzenden Nährlösungskomponente lässt sich mathematisch formulieren [9]. Ein kontinuierliches Fermentationssystem ist dementsprechend ebenfalls algebraisch beschreibbar. Bei Reinkulturen von Mikroorganismen unter genau bekannten Milieubedingungen kann man es auch zahlenmässig erfassen. Belebtschlammanlagen stellen nun allerdings äusserst komplexe Fermentationssysteme dar mit kompliziert zusammengesetzten Biozönosen und stets wechselnden Milieubedingungen. Betrachtet man den Belebtschlamm als einheitlichen "Organismus" und das zufliessende Abwasser als Nährmedium, in welchem eine gewisse Gruppe von organischen Verbindungen gesamthaft als "begrenzendes Substrat" wirksam ist (bei häuslichem Abwasser kann man wohl die Gesamtheit aller leicht fermentierbaren, organischen C-Verbindungen unter diesem Begriff zusammenfassen), so lassen sich im Prinzip die mathematischen Grundlagen der Durchlauffermentationen auch auf Belebtschlammsysteme anwenden. Man wird zwar kaum je in der Lage sein, eine allgemeingültige Berechnung einer Belebtschlammanlage nach diesen Gesetzmässigkeiten vorzuschlagen, da die notwendigen numerischen Basiswerte für jede Lokalität anders sind. Hingegen gestatten die Beziehungen, wenigstens jene Betriebsparameter und ihre inneren Zusammenhänge festzustellen, welche kausal den Gang einer Belebtschlammanlage bestimmen.

Eine seit längerer Zeit in Betrieb stehende Belebtschlammanlage stellt einen Beharrungszustand zwischen Organismenbestand (Schlammenge im 
ganzen System), Organismenproduktion (Úberschußschlammenge) und verbrauchtem Substrat (Reinigungswirkung) dar. Unter diesen Umständen lassen sich die wesentlichen Geschehnisse in einem Belüftungsbecken ("Reaktor") mit vier Grundgleichungen beschreiben:

a) Absolute Wachstumsgescbwindigkeit der Organismen. Jede lebende Zelle teilt sich in bestimmtem Rhythmus, d.h. die Wachstumsgeschwindigkeit der Population ist von der in jedem Zeitpunkt vorhandenen Zellenzahl abhängig:

$$
d x / d t=\mu x .
$$

Für die Zeit zur Verdoppelung der Zellenzahl ergibt sich beispielsweise $t_{d}=\ln 2 / \mu$.

b) Grösse der Gescbwindigkeitskonstanten $\mu$ für das.Wachstum. Sie ist von der Nährstoff konzentration abhängig, wobei man annehmen darf, dass in einer Nährlösung in der Regel nur ein einziger Faktor wachstumsbegrenzend wirkt (irgendeine physiologisch unentbehrliche und sich vor allen anderen Nährstoffen im Milieu erschöpfende Verbindung). MONOD [9] hat experimentell diesen Zusammenhang in Analogie zur Geschwindigkeitskonstanten enzymatischer Reaktionen (Michaelis-Menten-Gleichung) formulieren können:

$$
\mu=\mu_{m} \frac{s}{K_{s}+s} .
$$

$K_{s}$ ist die Substratkonzentration, bei welcher die Wachstumsgeschwindigkeit der halben maximalen Wachstumsgeschwindigkeit $\mu_{m} / 2$ entspricht. SCHULZE und LIPE [I2] empfehlen, diese Gleichung durch jene von TEISSIER [I4], $\mu=\mu_{m}\left(\mathrm{I}-e^{-c s}\right)$, zu ersetzen, da sie den experimentellen Beobachtungen besser gerecht werde ( $c=$ Konstante). Abgesehen davon, dass es für unsere Betrachtung gleichgültig ist, welche der beiden Beziehungen gewählt wird, bevorzugen wir aus physiologischen Überlegungen vorläufig den Ansatz von MoNOD.

c) Substratausnützung für den Zellaufbau. In jede neugebildete Zelle ist eine bestimmte, gleiche Menge des begrenzenden Substrats für die Körpersubstanz investiert worden:

$$
\left(x-x_{0}\right)=Y_{S}\left(s_{R}-s\right) .
$$

Der "Ausnützungsfaktor" $Y_{S}$ bezieht sich nur auf den Substratverbrauch für die Neubildung von Zellsubstanz, nicht aber für den Konsum von Substrat, das laufend im Energiestoffwechsel veratmet wird. Der totale Sub- 
stratverbrauch des Schlamms ist die Summe aus dem investierten und veratmeten Substrat:

$$
\Delta s_{\text {total }}=\Delta s_{\text {invest. }}+\Delta s_{\text {respiriert }} .
$$

d) Geschmindigkeit der Substratinvestition: Sie ergibt sich aus der Wachstums geschwindigkeit und der Substratinvestition (Gl. I, 2 und 3) zu:

$$
-Y_{S} d s / d t=d x / d t=\mu x
$$

oder

$$
\left(s_{R}-s\right)=\mu \frac{x}{Y_{S}} t \text {, }
$$

bzw.

$$
\left(s_{R}-s\right)=\mu_{m} \frac{s}{K_{s}+s} \frac{x}{Y_{S}} t .
$$

e) Gescbmindigkeit der Substratelimination: Wir haben in früheren Untersuchungen (WUHRMANN und v.BEUST [2I] nachgewiesen, dass innerhalb der analytisch zugänglichen Grenzen eine reine Substanz (Substrate im Sinne der Physiologie können nur definierte Verbindungen sein, summarische Analysengrössen wie org. $\mathrm{C}$, org. $\mathrm{N}$ oder gar der $\mathrm{BSB}_{5}$ können nicht gesamthaft als definiertes Substrat bezeichnet werden) mit annähernd konstanter Geschwindigkeit aus dem Abwasser verschwindet, wobei die Eliminationsgeschwindigkeit proportional zur Schlammkonzentration, aber unabhängig von der Substratkonzentration ist:

bzw.

$$
\begin{aligned}
\frac{d s}{d t} & =-\varepsilon x, \\
s_{R}-s & =\varepsilon \times t .
\end{aligned}
$$

Bei Gleichsetzung von 4 a und 5 ergibt sich für die "Konstante" $\varepsilon$ (= spezifische Eliminationsleistung):

$$
\varepsilon=\frac{\mathrm{I}}{Y_{S}} \mu_{m} \frac{s}{K_{s}+s} .
$$

Der Wert $\varepsilon$ kann offenbar nur in jenem Bereich annähernd konstant sein, in welchem die Grösse der Halbwertskonzentration $K_{s}$ im Vergleich zu s sehr klein ist. Soweit unsere Kenntnisse heute reichen, ist für Substrate, die als wachstumsbegrenzend in Frage kommen, der Wert von $K_{s}$ tatsächlich gering und liegt sogar für Kohlenstoffquellen in der Grössenordnung von $\mu \mathrm{mol} / \mathrm{l}$. Beziehung ( $\mathrm{s}$ ) gibt somit eine plausible Erklärung für die beobachtete lineare Elimination eines Substrats in Funktion der Zeit.

2.2 Belüftungsbecken als vollständig durchmischte "Reaktoren"

In den Belüftungsbecken unserer Versuchsanlage (Länge etwa $3 \mathrm{~m}$ ) waren der Einlauf für das Rohwasser mit dem Rücklaufschlamm und der Auslauf an entgegengesetzten Enden angeordnet. Das kurze Becken und die 
grosse Rücklaufrate (in der Regel $100 \%$ ) bewirkten aber, dass man trotz Längsdurchfluss mit guten Gründen die Becken als vollständig durchmischte Reaktoren bezeichnen kann. Anhand der Grundgleichungen (I-4) kann man ein Belebtschlammsystem mit Rücklauf, vollständig durchmischtem Belüftungsbecken und einem Nachklärbecken wie folgt beschreiben (wir halten uns didaktisch an die einfache Darstellung von Herbert [s]).

Zunächst ist noch festzuhalten, dass sich die Schlammengen (Zellen) in den $\mathrm{Zu}$ - und Abflüssen von Belüftungs- und Nachklärbecken wie folgt verhalten müssen:

Belüftungsbecken: $\quad \frac{\text { Zellzufluss }}{\text { Zellabfluss }}=\frac{a c}{(\mathbf{I}+a)}$,

Nachklärbecken: $\quad \frac{\text { Zellzufluss }}{\text { Zellabfluss }}=\mathrm{I}=\frac{(\mathrm{I}+a) f x_{1}}{a f x_{1} c+f x_{2}}$,

bzw.

$$
\frac{x_{2}}{x_{1}}=\mathrm{I}+a-a c=A \text {. }
$$

Man kann nun folgende Bilanzverhältnisse für das Belüftungsbecken anschreiben:

a) Zellen(Scblamm-)bilanz:

Schlammzunahme $=$ Zufluss mit RS + Zuwachs - Abfluss

$$
f^{d x_{1}} \frac{f a C x_{1}}{d t}=\quad+x_{1} \mu_{m} \frac{s}{k_{s}+s}-(\mathrm{I}+a) f x_{1} .
$$

Im Beharrungszustand des kontinuierlichen Betriebes, d. h. bei konstantem Schlammgehalt im Becken (wenn also $d x_{1} / d t=0$ ist), so wird:

$$
\mu_{m} \frac{s}{K_{s}+s}=f A \text {. }
$$

b) Substratbilanz:

Substratänderung $=$ Zufluss - Verbrauch - Abfluss

$$
f_{d t}^{d s}=f s_{R}+a f s-x_{1} \frac{\mathrm{I}}{Y_{S}} \mu_{m} \frac{s}{K_{s}+s}-(\mathrm{I}+a) f s .
$$

Bei konstantem Betrieb ist wieder $d s / d t=0$, so dass man mit Hilfe von (6) und (9) erhält

oder

$$
\begin{gathered}
s=s_{R}-\frac{x_{1}}{Y_{S}} A \\
\left(s_{R}-s\right)=x_{1} A \\
Y_{S}
\end{gathered} .
$$


Da man den Reinigungseffekt $\eta$ definiert als $\left(s_{R}-s\right) / s_{R}$, kann man ihn mit (II a) formulieren:

$$
\frac{s_{R}-s}{s_{R}}=\eta=\frac{x_{1}}{s_{R}} \frac{A}{Y_{S}} .
$$

Nun ist aber definitionsgemäss die Schlammbelastung $S_{b}=R_{h} s_{R} / x_{1}$, so dass sich für den Zusammenhang zwischen Reinigungseffekt und Schlammbelastung

ergibt.

$$
\eta=\frac{R_{h}}{S_{b}} \frac{A}{Y_{S}}
$$

Gleichung (I2a) führt zu folgenden Feststellungen:

Der Begriff der Schlammbelastung ist kausal gerechtfertigt, er steht in unmittelbarer Beziehung zum Reinigungseffekt. Nur die im Beluiftungsbecken vorhandene Schlammenge ist für die Berechnung der Schlammbelastung massgebend.

Der Reinigungseffekt ist kausal von der hydraulischen Raumbelastung (bzw. der Belüftungszeit, berechnet für Abwasser allein) bei gegebener Schlammkonzentration und Zuflusskonzentration abhängig. Da der Quotient $A / Y_{S}$ keine konstante Grösse darstellt, weil $A$ mit der Wirkung des Nachklärbeckens variiert und $Y_{S}$ von zahlreichen Faktoren beeinflusst wird (Temperatur, Qualität des Rohwassers usw.), ist $\eta$ nicht allein von der Raumbelastung, bzw. Schlammbelastung abhängig. Ferner ist als äusserst wichtiger Punkt zu berücksichtigen, dass die Beziehung (I2 a) nur gültig sein kann, wenn eine gleichbleibende, spezifische Eliminationsleistung (g Substrat/g Trockensubstanz $\cdot h$ ) des Belebtschlamms vorausgesetzt ist. Wie noch zu zeigen sein wird, ist aber gerade diese Grundbedingung bei Belebtschlammanlagen nicht erfüllt. Die Eliminationskonstante $\varepsilon$ (Gl. sa) des Schlamms erweist sich nämlich als von der Schlammbelastung abhängig.

c) Überschußschlammproduktion:

Aus (6) und (I r a) ergibt sich für die Konzentration der aus dem System abfliessenden Zellmenge (man muss sie sich im gesamten abfliessenden Wasser suspendiert denken):

$$
x_{2}=Y_{S}\left(s_{R}-s\right) \quad\left[\mathrm{g} / \mathrm{m}^{3} \text { Abwasser }\right] \text {. }
$$

Die Angabe der Úberschußschlammproduktion pro $\mathrm{m}^{3}$ verarbeitetes Rohwasser ist also sinnvoll. Multipliziert man Gleichung (I3) mit der hydraulischen Raumbelastung

$$
R_{h} x_{2}=Y_{S} R_{h}\left(s_{R}-s\right) \quad\left[\mathrm{g} / \mathrm{m}^{3}{ }_{B B} \cdot \mathrm{Tag}\right],
$$


so erhält man die tägliche Überschußschlammenge pro $\mathrm{m}^{3}$ Belüftungsraum in Ahbängigkeit von der Abbauleistung der Anlage pro $\mathrm{m}^{3}$ Belüftungsraum.

Der Ausniitzungsfaktor $Y_{S}$ ist offensichtlich eine wichtige Bestimmungsgrösse für die Berechnung des Úberschußschlammanfalls, und man sollte bei Experimenten anstreben, seinen Wert in Abhängigkeit von den Betriebsparametern festzuhalten.

Aus Gl. (6) und der Definition für die Schlammbelastung kann man auch eine Beziehung zwischen Schlammbelastung und Überschußschlammenge ableiten :

$$
x_{2}=\frac{R_{h}}{S_{b}} s_{R} A \quad\left[\mathrm{~g} / \mathrm{m}^{3} \text { Rohwasser }\right],
$$

die sofort zeigt, dass bei konstanter Belüftungszeit und Substratkonzentration und bei gleichbleibendem Konzentrierungseffekt im Nachklärbecken, die Überschußschlammenge pro Kubikmeter Rohwasser theoretisch umgekehrt proportional zur Schlammbelastung sein müsste.

In allen vorstehenden Gleichungen kommen zwei wesentliche Elemente bei ihrer Anwendung auf Belebtschlammsysteme nicht zum Ausdruck:

a) Der Gehalt des Abwassers an primären Schwebestoffen: sofern diese in unveränderter Form und quantitativ in den Überschußschlamm übergehen würden, könnten sie einfach zu dem "echten" Zuwachs des Belebtschlamms, wie er aus den vorstehenden Bedingungen errechnet werden kann, zugeschlagen werden. Wie wir später zeigen, ist diese Annahme aber keineswegs statthaft (wenigstens bei häuslichem Abwasser), weil die primären Schwebestoffe (soweit sie biologisch angreif bar sind) ebenfalls in den Stoffumsatz des Systems einbezogen werden.

b) Die Autolyse des Belebtschlamms: Die Betriebsbedingungen aller Abwasserreinigungsanlagen für häusliches und der meisten Anlagen für industrielles Abwasser sind gekennzeichnet durch die sehr geringe Konzentration des ausnützbaren Substrats für die Schlammorganismen. Da zum Abbau der im Abwasser vorkommenden, langsam angreif baren Substanzen trotzdem verhältnismässig lange Belüftungszeiten und hohe Schlammkonzentrationen notwendig sind, muss ein Belebtschlammsystem notwendigerweise mit Schlammrückführung betrieben werden. Dies hat aber zur Folge, dass eine Organismenmasse im System akkumuliert wird, für welche eine ausreichende Ernährungsbasis gar nicht vorhanden ist, so dass laufend ein mehr oder weniger grosser Anteil der zirkulierenden Zellen abstirbt und der Autolyse verfällt. Die gelösten Autolyseprodukte werden im wesentlichen sofort wieder als Substrat ausgenützt, während sich ungelöstes Material (vor allem die Zellmembranen) anreichert. Die in Gl. (13) angegebene Be- 
ziehung, die nur für Systeme gilt, in welchen dauernd ein Substratüberschuss vorhanden ist, muss deshalb für den Belebtschlammbetrieb modifiziert werden. Wir kommen auf dieses Problem in Abschnitt 5 noch eingehend zurück.

Die Beziehungen zwischen dem Reinigungseffekt einer Belebtschlammanlage und ihren Betriebsbedingungen sind schon häufig Gegenstand von experimentellen Untersuchungen und Auswertungen praktischer Betriebsbeobachtungen gewesen. Wir werden auf einzelne dieser Arbeiten bei der Besprechung der Versuchsergebnisse zurückkommen. Leider ist meist die Variationsbreite der untersuchten Parameter verhältnismässig eng gewesen und ferner wurden die eventuellen Wechselwirkungen zwischen einzelnen Betriebsbedingungen nicht geprüft.

Für die eigenen Untersuchungen haben wir uns deshalb zum Ziel gesetzt, die Beziehungen zwischen dem Reinigungseffekt einer Belebtschlammanlage und ihrer hydraulischen Raumbelastung, der Schlamm- und Sauerstoffkonzentration sowie der Temperatur im Belüftungsbecken innerhalb eines breiten Gebietes abzuklären, wobei sowohl die Einzelwirkungen dieser unabhängigen Variablen sowie vor allem auch ihre Wechselwirkungen systematisch beobachtet werden sollten. Den Versuchen wurde eine ausgedehnte Liste von Versuchsfragen zugrunde gelegt, die auch eine biologische Charakterisierung der Belebtschlämme und eine biologische Prüfung der Auswirkungen verschieden gereinigter Abwässer in einer fliessenden Vorflut einschlossen. Nachfolgend werden nur jene Ergebnisse herausgegriffen, die sich auf die Elimination organischer Inhaltsstoffe des Abwassers sowie auf die Bildung von Überschußschlamm beziehen.

\section{Experimentelle Grundlagen}

\subsection{Versucbsplan}

Für die nachfolgende Interpretation der Experimente ist es entscheidend wichtig, dass sie konsequent auf Grund eines statistischen Plans ausgeführt wurden. Der Gedankengang war der folgende: Das gestellte Problem ist nur lösbar, wenn die 3 Betriebsparameter in verschiedenen Grössenstufen geprüft werden. Es sind daher eine grössere Zahl von Einzelversuchen durchzuführen, die sich naturgemäss über einen längeren Zeitraum verteilen. Es ist damit zu rechnen, dass sowohl die Abwasserkonzentration als auch zahlreiche äussere Einflüsse, die nicht näher bezeichnet werden können, auf das Ergebnis einwirken. Die Versuche müssen also derart angeordnet werden, dass alle Streuungskomponenten, die nicht unabhängige 
Versuchsvariable sind, sich in zufälliger Weise über das ganze Experiment verteilen und damit bei der statistischen Auswertung mit der gesamten Reststreuung innerhalb des Versuchsmaterials erfasst werden.

Wir wählten folgende Stufen für die unabhängigen Versuchsvariablen (exakte Werte vgl. Tab. 18-20): hydraulische Raumbelastung etwa 10, 30 und $60 \mathrm{~m}^{3} / \mathrm{m}^{3}$. Tag; Schlammkonzentration im Belüftungsbecken etwa 600, 3300 und $6000 \mathrm{mg}$ TS $/ 1 ; \mathrm{O}_{2}$-Gehalt im Belüftungsbecken etwa I, 4 und $7 \mathrm{mg}$ $\mathrm{O} / 1$; Temperatur im Belüftungsbecken $\left\langle\mathrm{II}^{\circ} \mathrm{C},>\mathrm{I}_{3}{ }^{\circ} \mathrm{C}\right.$.

Es wurden alle Kombinationen der Grössenstufen geprüft, d.h. es waren $3^{3} \cdot 2=54$ Einzelversuche vorzunehmen. Um dieses Programm in absehbarer Zeit zu verwirklichen, bildeten wir aus den vorhandenen Baukörpern unserer Versuchsstation 3 Parallelanlagen, welche die gleichzeitige Durchführung von 3 Experimenten erlaubten: Die Einzelversuche (je eine Kombination von unabhängigen Variablen umfassend) wurden nach einem $\mathrm{Zu}-$ fallsschema in den Zeitplan eingestellt. Die theoretische Grundlage für eine solche Versuchsanordnung ist in Statistiklehrbüchern nachzulesen und kann hier nicht behandelt werden. Wesentlich ist dabei folgendes: Zufällige Variabilitäten, die auf das Ergebnis aller Einzelversuche einwirken, können normalerweise die Wirkung der unabhängigen Versuchsvariablen kaum verdecken, da ja Versuche mit gleicher Grösse einer einzigen Variablen neunmal wiederholt sind (bei Beriicksichtigung beider Temperaturblöcke sogar achtzehnmal) und alle Kombinationen von zwei gleichzeitig variierten Faktoren in 3 Wiederholungen (in beiden Temperaturblöcken zusammen 6 Wiederholungen) vorkommen, usw. Hingegen liegen keine Wiederholungen vor für die Einzelexperimente mit der Kombination von 3 (mit Temperatur: 4) Variablen. Man kann deshalb Einzelversuche nicht miteinander in allen Details vergleichen, da sie bezüglich Zufallsvariabilitäten stark verschieden beeinflusst sein können.

\subsection{Versucbseinricbtungen}

Die in das Experiment einbezogenen Anlagen auf unserer Versuchsstation Tüffenwies sind schon früher beschrieben worden (WUHRMANN [I8]) und wir verweisen auf diese Darstellung. Die Zusammenschaltung der Bauteile und wesentliche hydraulische Betriebsdaten für das vorliegende Experiment gehen aus der Tab. I hervor.

Das rohe Abwasser für die Versuche wurde aus dem städtischen Hauptsammler in unsere Vorkläranlage gefördert und unterlag naturgemäss hinsichtlich Zusammensetzung und Konzentration den üblichen täglichen und jahreszeitlichen Schwankungen. Die Versuchsanordnung hat aber diese 
Tabelle 1

Dimensionen und mittleve Betriebsbedingungen der Versuchseinrichtungen

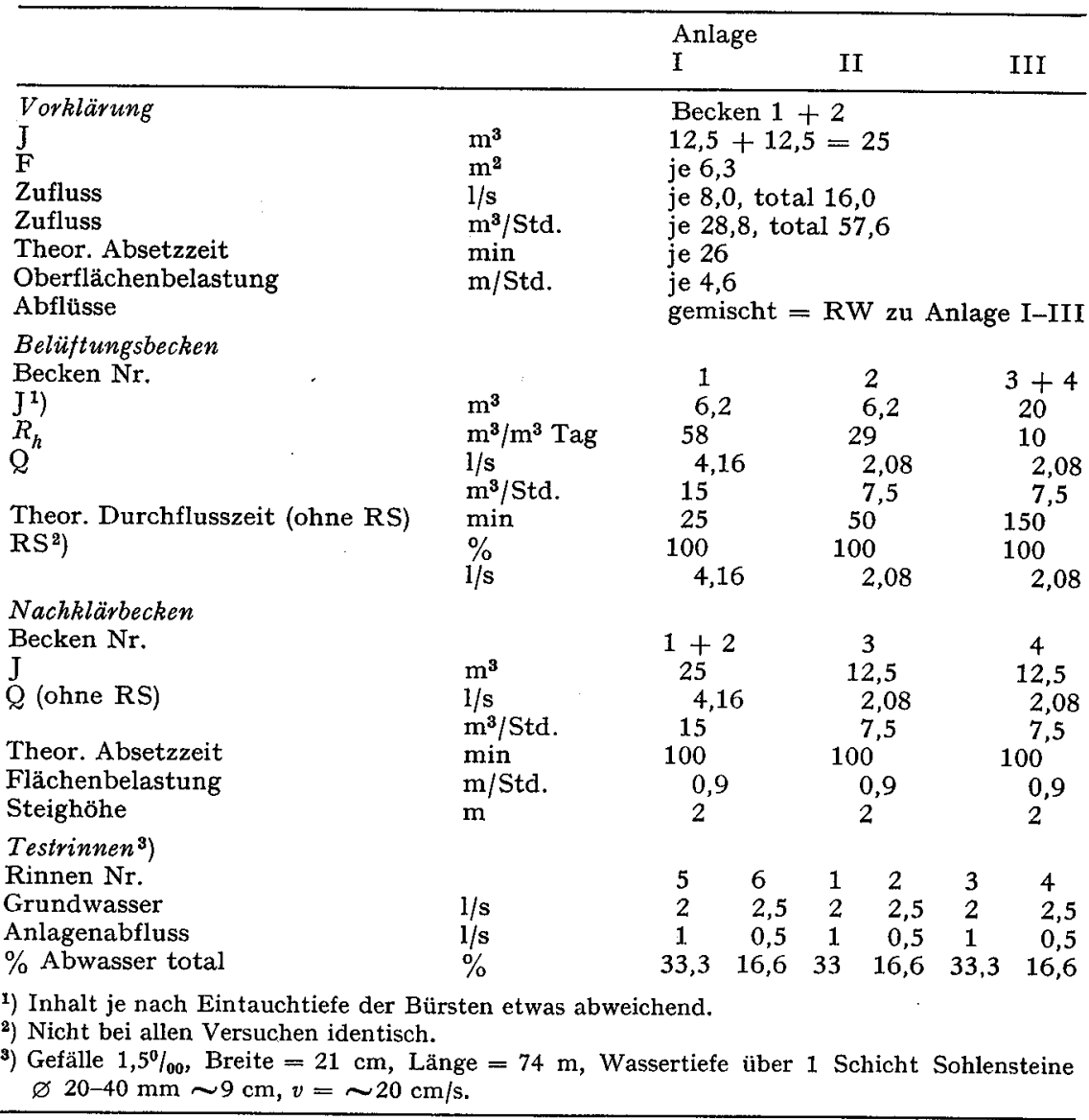

Variabilität in zufälliger Weise auf das ganze Versuchsmaterial verteilt, so dass sie bei der Auswertung nicht weiter berücksichtigt werden muss. Hingegen ist darauf hinzuweisen, dass die verhältnismässig geringeAbwasserkonzentration (spez. Abwassermenge rd. 400 1/E Tag!) eine gewisse Beschränkung der Information mit sich bringt. Theoretisch ist wohl eine Extrapolation auf andere Substratkonzentrationen möglich, praktisch dürfte aber in Anbetracht der komplexen Zusammensetzung von Abwasser einige Vorsicht geboten sein. Unsere Ergebnisse gelten bezüglich ihrer absoluten Werte deshalb nur für ein Abwasser überwiegend häuslichen Charakters von eher geringer Konzentration. 


\subsection{Versucbsdurcbfübrung}

Entsprechend der Forderung zur Untersuchung von zwei verschiedenen Temperaturbereichen gliederten sich die Experimente in zwei Gruppen zu $3^{3}$ identischen Versuchen, die in jenen Winter-, bzw. Sommermonaten der Jahre 1957-196I ausgeführt wurden, in welchen die verlangten Temperaturgrenzen in den Belüftungsbecken dauernd eingehalten werden konnten. Die relativ kurzen Perioden mit tiefen Abwassertemperaturen bedingten, dass sich speziell die Experimente im Temperaturbereich $\angle \mathrm{II}{ }^{\circ} \mathrm{C}$ über 4 Winter erstreckten, während die "Warm"wasserversuche sich innerhalb drei Sommern durchbringen liessen.

Jeder Versuch dauerte 6-8 Wochen und gliederte sich in 2 Perioden:

a) Vorperiode: Einstellung und Einfahren der Anlage bei den geforderten Bedingungen. Dauer mindestens 3-4 Wochen.

b) Analysenperiode: Anlage im Beharrungszustand bezüglich der unabhängigen Variablen. Dauer mindestens 3-4 Wochen (oder bis die 9 verlangten Probenahmen für die chemische Untersuchung durchgeführt waren).

Die Anlagen wurden ohne Unterbruch von einem Experiment ins nächste übergeführt.

\subsection{Versuchsbeobacbtungen}

Die Kontrolle der Experimente erfolgte einerseits mit den täglichen Betriebsbeobachtungen an den Versuchsanlagen (Messung der Grösse der unabhängigen Variablen, UUberschußschlammproduktion, Absetzeigenschaften des Schlamms usw.) und andererseits durch die Analyse von Proben des rohen Abwassers (Abfluss der Vorklärung) und der Anlagenabflüsse während der Analysenperiode. Die Probenahmen erfolgten mit automatischem Gerät alle ro min in Probenahmeflaschen, die in einem Tiefkühler standen, so dass jedes Probeninkrement sofort gefror. Die 24stündigen Sammelproben gelangten noch in tiefgefrorenem Zustand ins Laboratorium und wurden erst unmittelbar vor der Untersuchung aufgetaut. Probenahmezeit $08 \mathrm{~h}-08 \mathrm{~h}$. In jeder Analysenperiode wurden ferner von allen Belebtschlämmen dreimal die endogene Atmung (Schlamm gewaschen) und die Substratatmung nach Zusatz eines doppeltkonzentrierten Rohwassers gemessen. Ferner erfolgten 3 eingehendere mikroskopische Untersuchungen mit Auszählung der Protozoen und anderer gut erkennbarer Belebtschlammanteile.

Die chemische Untersuchung umfasste beim Rohwasser und den Anlagenabflüssen Schwebestoffe, Oxydierbarkeit, $\mathrm{BSB}_{5}$, org. $\mathrm{C}$, org. $\mathrm{N}, \mathrm{NO}_{2}^{-}-\mathrm{N}$, 
$\mathrm{NO}_{3}^{-}-\mathrm{N}, \mathrm{NH}_{4}^{+}-\mathrm{N}$, Gesamt-P, $\mathrm{Cl}^{\prime}$. Die verwendeten Analysenmethoden sind in Tab. 2 zusammengefasst.

Die Chloride wurden als inerte Tracersubstanz beobachtet, um zu kontrollieren, ob die Sammelproben der Anlagenabflüsse mit der Sammelprobe des Rohwassers korrespondierten.

Tabelle 2

Ubersicht der verwendeten Analysenverfahren

\begin{tabular}{|c|c|}
\hline Komponente & Methode \\
\hline Schwebestoffe & $\begin{array}{l}\text { gravimetrisch nach Filtration durch Membranfilter, Methode } \\
\text { EAWAG (nicht veröffentlicht) }\end{array}$ \\
\hline $\mathrm{BSB}_{5}$ & $\begin{array}{l}\text { Standard Methods for the Examination of Water, Sewage and } \\
\text { Industrial Wastes, } 1955\end{array}$ \\
\hline org. C, Abwasser & $\begin{array}{l}\text { anfänglich nach van SLYKE und FoLCH }[13] \text {, später mikro- } \\
\text { manometrisch nach Persulfatoxydation, Methode EAWAG } \\
\text { (nicht veröffentlicht) }\end{array}$ \\
\hline org. C, Schlämme & $\begin{array}{l}\text { anfänglich nach VAN SLYKE und FoLCH [13], später trockene } \\
\text { Verbrennung und gasometrische } \mathrm{CO}_{2} \text {-Best. nach STRÖHLEIN }\end{array}$ \\
\hline $\operatorname{org} . \mathrm{N}$ & nach KJELDAHL \\
\hline $\mathrm{NH}_{4}^{+}$ & dest. nach KJELDAHL \\
\hline $\mathrm{NO}_{2}^{-}$ & nach GrIEs, JLosvay, Schweiz. Lebensmittelbuch 1934 \\
\hline $\mathrm{NO}_{3}^{-}$ & nach ScHERINGA, Schweiz. Lebensmittelbuch 1934 , modifiziert \\
\hline Gesamt-P & $\begin{array}{l}\text { im Kjeldahl Aufschluss nach Frske und SubBarow (J. biol. } \\
\text { Chem. } 66,375,1925 \text { ), modifiziert }\end{array}$ \\
\hline $\mathrm{K}$ & flammenphotometrisch \\
\hline $\mathrm{Cl}^{-}$ & mercurometrisch nach Standard Methods \\
\hline
\end{tabular}

\subsection{Statistische Ausmertung der Beobachtungen}

Von allen Beobachtungen eines Versuches (Betriebsbeobachtungen : 25 bis 35 Einzelmessungen pro Parameter; analytische Untersuchungen: grundsätzlich 9 Tagesmittelwerte pro Versuch) wurden die Mittelwerte und ihre Standardabweichungen berechnet. Für die beiden Temperaturbereiche konnten sodann Sammeltabellen mit 3 Eingängen für die unabhängigen Variablen Raumbelastung, Schlammkonzentration und Sauerstoffkonzentration erstellt werden. Die Urteilsbildung erfolgte anschliessend an Hand einer Varianzanalyse sowohl für die 54 Versuche gesamthaft als auch für die 27 Sommer- und 27 Winterversuche getrennt. Für die weitere rechnerische Auswertung konnten dann Sammelwerte benützt werden, wenn sich ergab, dass einzelne Faktoren keinen statistisch gesicherten Einfluss auf die Beobachtungen ausuibten. Aus verschiedenen Gründen musste leider bei der Endauswertung aller Versuche ein Experiment im Block der niedrigen Temperaturen (29/3300/4) fallengelassen werden. Dort, wo die statistische Auswertung einen vollständigen Satz von Beobachtungen verlangte, wurde 
dieser fehlende Wert rechnerisch als wahrscheinlichstes Resultat nach der Technik der Missing Values (BRownLEE [I]) geschätzt.

\subsection{Versucbsbeobacbtungen}

In den Tab. I 8-34 im Anhang sind einige wesentliche Versuchsdaten und Befunde zusammengefasst. Die Bezeichnung der Tabelleneingänge für hydraulische Raumbelastung, Schlammkonzentration und Sauerstoff konzentration im Belüftungsbecken erfolgte mit runden Ziffern gemäss Versuchsplan. Für die rechnerische Auswertung wurden selbstverständlich die in den einzelnen Experimenten effektiv gemessenen Werte verwendet. Dort, wo es notwendig schien, ist bei jedem Versuch die Standardabweichung des in die Tabelle aufgenommenen Mittelwertes der Beobachtungen angegeben. Wir haben die Auswahl der Tabellen nach den Notwendigkeiten dieses Berichtes getroffen. Weitere Angaben werden wir in zusätzlichen Veröffentlichungen über diesen Versuch bringen.

Entsprechend der Versuchsplanung erfolgt die Prüfung der Wirkungen verschiedener Stufen der Variablen anhand der Summen jener Tabellenwerte, welche zu den in den Vergleich einbezogenen Versuchen gehören. Tab. 3 gibt ein Beispiel für diese erste Auswertungsstufe.

Aus den Tab. I 8-20 (Anhang) geht hervor, dass die drei unabhängigen Variablen, hydraulische Raumbelastung, Schlammkonzentration und Sauerstoffkonzentration mit ziemlich guter Genauigkeit bei allen Experimenten eingehalten werden konnten. Grössere Variabilitäten ergaben sich bezüglich der Temperatur innerhalb der beiden Temperaturblöcke, und leider beträgt die mittlere Temperaturdifferenz zwischen den Blöcken nur $6,3^{\circ} \mathrm{C}$. Trotzdem kamen nach unserer Auffassung alle wesentlichen Temperaturabhängigkeiten zum Ausdruck. Wie die Tabellen der Konzentration einzelner Abwasserkomponenten zeigen, traten innerhalb der 4 Versuchsjahre erwartungsgemäss sehr grosse Unterschiede bei den einzelnen Experimenten auf. Auch innerhalb eines Experiments ergaben sich oft beträchtliche Schwankungen zwischen einzelnen Probenahmetagen (z.B. Regenwettertage, vgl. Streuung der Mittelwerte). Die Variabilität der Abwasserzusammensetzung stellt denn auch bei weitem die grösste Komponente der Gesamtstreuung im Gesamtversuch und der Reststreuung beim Vergleich einzelner Hauptwirkungen und Wechselwirkungen dar. Soviel wir beurteilen können, vermag sie aber keine der uns wesentlich erscheinenden Beziehungen zu verdecken, obschon gelegentlich einzelne Mittelwerte erheblich vom Erwartungswert abweichen. Bei der Gesamtbeurteilung kommen wir jedenfalls zur Schlussfolgerung, dass die angewendete Me- 
thode der zufälligen Verteilung der Experimente innerhalb der Beobachtungsperiode ein durchaus taugliches Mittel darstellt, um eine grössere Zahl von Versuchen nacheinander durchzuführen und trotz der unvermeidlichen Streuungen der Versuchsbedingungen mindestens die Hauptwirkungen von unabhängigen Variablen zuverlässig erfassen zu können.

\section{Einfluss von Temperatur, Raumbelastung, Schlamm- konzentration und Sauerstoffspannung im Belüftungsbecken auf die Elimination organischer Verunreinigungen, gemessen am organischen Kohlenstoff, organischen Stickstoff und $\mathrm{BSB}_{5}$}

Wir werden nachstehend die Versuchsergebnisse bezüglich der Reini-

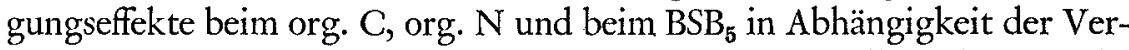
suchsvariablen prüfen. Zur Illustration soll dabei ein vollständiges Beobachtungsmaterial für den org. $C$ dargestellt werden, während wir bei den andern Komponenten nur die zum Beleg unerlässliche, statistische Auswertung in Form der Varianzanalyse geben. Die Interpretation der Beobachtungen erfolgt in einem späteren Abschnitt.

\subsection{Elimination von organischen Koblenstoffverbindungen}

Von sämtlichen gefassten Proben der Anlagenzu- und -abflüsse sowie von allen Úberschußschlammproben wurde der Gehalt an org. C (jede Probe 3 fach) bestimmt. Wir legten besonderen Wert auf diese Analyse, weil der org. C zurzeit die einzige, verhältnismässig sinnvolle und gutbestimmbare Masszahl für den Gehalt eines Abwassers an organischen Verunreinigungsstoffen darstellt. In Tab. 3 sind die Mittelwerte aus den 9 Tagesbeobachtungen des Reinigungseffektes in jedem Versuch für die beiden Temperaturserien zusammengestellt. Ferner sind auch die für die Varianzanalyse benötigten Summen der Beobachtungswerte für die den einzelnen Kombinationen von Variablen zugehörigen Gruppen von Versuchen angegeben. Diese Summen geben schon ohne weitere Rechnung einen Hinweis auf die Abhängigkeiten, denen der Reinigungseffekt unterliegt.

In den nachfolgenden Tabellen der Varianzanalysen verwenden wir folgende Bezeichnungen:

Fg Freiheitsgrade

SQ Summe der Quadrate

DQ Durchschnittsquadrate

F Quotient aus Durchschnittsquadrat einer Variablen und dem Durchschnittsquadrat der Reststreuung

P Wahrscheinlichkeit des Wertes F gemäss F-Verteilung, wobei 
$P \leqslant 0$, or $(\geqslant 99,9 \%)$ sehr gut gesichert,

$P \leqslant 0,05(\geqslant 95 \%)$ gut gesichert,

$P \leqslant 0, I \quad(\geqslant 90 \%)$ gesichert,

P $>0, I \quad(<90 \%)$ nicht gesichert.

In Anbetracht der grossen, unvermeidlichen Variabilitäten in einem solchen Experiment begnügen wir uns mit einer Wahrscheinlichkeit von $\mathrm{P} \leqslant 0, \mathrm{I}(90 \%)$, um ein Resultat als schlüssig zu bezeichnen. Dieselbe Wahrscheinlichkeit verwenden wir auch für die Berechnung der Vertrauensgrenzen von Mittelwerten.

Tabelle 3

Statistische Auswertung $\eta \%$ org. $C$

\begin{tabular}{|c|c|c|c|c|c|c|c|c|c|c|c|}
\hline \multicolumn{6}{|c|}{ Temperatur $>13^{\circ} \mathrm{C}$} & \multicolumn{6}{|c|}{ Temperatur $<11^{\circ} \mathrm{C}$} \\
\hline $\begin{array}{l}\mathrm{O}_{2} \\
\mathrm{mg} / 1\end{array}$ & $\begin{array}{l}\text { Schlamm- } \\
\text { l konzen- } \\
\text { tration } \\
\mathrm{mg} / \mathrm{l}\end{array}$ & $\begin{array}{l}\text { Hydra } \\
\text { Raum } \\
\mathrm{m}^{3} / \mathrm{m}^{3} \\
58\end{array}$ & $\begin{array}{l}\text { aulisch } \\
\text { abelastı } \\
\text {. Tag } \\
29\end{array}$ & $\begin{array}{l}\text { he } \\
\text { tung } \\
9\end{array}$ & $\mathrm{~S}$ & $\begin{array}{l}\mathrm{O}_{2} \\
\mathrm{mg} / \mathrm{l}\end{array}$ & $\begin{array}{l}\text { Schlamm- } \\
1 \text { konzen- } \\
\text { tration } \\
\mathrm{mg} / 1\end{array}$ & & $\begin{array}{l}\text { aulische } \\
\text { ibelastun } \\
\text {. Tag } \\
29^{9} 9\end{array}$ & $\begin{array}{l}\text { ung } \\
9\end{array}$ & $S$ \\
\hline 1 & $\begin{array}{r}600 \\
3300 \\
6000\end{array}$ & $\begin{array}{l}49,0 \\
71,4 \\
74,5\end{array}$ & $\begin{array}{l}63,5 \\
79,5 \\
71,0\end{array}$ & $\begin{array}{l}64,0 \\
85,4 \\
85,0\end{array}$ & $\begin{array}{l}176,5 \\
236,3 \\
230,5\end{array}$ & 1 & $\begin{array}{r}600 \\
3300 \\
6000\end{array}$ & $\begin{array}{l}30,4 \\
64,5 \\
67\end{array}$ & $\begin{array}{l}46,2 \\
72,4 \\
71,7\end{array}$ & $\begin{array}{l}57,7 \\
81,3 \\
78,5\end{array}$ & $\begin{array}{l}134,3 \\
218,2 \\
217,2\end{array}$ \\
\hline $\mathrm{S}$ & & 194,9 & 214,0 & 234,4 & 643,3 & $\mathrm{~S}$ & & 161,9 & 190,32 & 217,5 & 569,7 \\
\hline \multirow{2}{*}{$\begin{array}{l}4 \\
\mathrm{~S}\end{array}$} & $\begin{array}{r}600 \\
3300 \\
6000\end{array}$ & $\begin{array}{l}70,0 \\
70,0 \\
75,8\end{array}$ & $\begin{array}{l}75,4 \\
81,5 \\
83,7\end{array}$ & $\begin{array}{l}66,7 \\
80,4 \\
86,4\end{array}$ & $\begin{array}{l}212,1 \\
231,9 \\
245,9\end{array}$ & 4 & $\begin{array}{r}600 \\
3300 \\
6000\end{array}$ & $\begin{array}{l}38,8 \\
59,5 \\
65,8\end{array}$ & $\begin{array}{l}53,2 \\
70 \\
71,2\end{array}$ & $\begin{array}{l}60,0 \\
79,1 \\
79,0\end{array}$ & $\begin{array}{l}152,0 \\
208,6 \\
216,0\end{array}$ \\
\hline & & 215,8 & 240,6 & 233,5 & 689,9 & $\mathrm{~S}$ & $S$ & 164,1 & 194,42 & 218,1 & 576,6 \\
\hline \multirow{4}{*}{$\begin{array}{l}S \\
S \\
S \\
S\end{array}$} & $\begin{array}{r}600 \\
3300 \\
6000\end{array}$ & $\begin{array}{l}61,8 \\
75,4 \\
78,9\end{array}$ & $\begin{array}{l}65,4 \\
78,0 \\
79,8\end{array}$ & $\begin{array}{l}70,4 \\
79,0 \\
83,6\end{array}$ & $\begin{array}{l}197,6 \\
232,4 \\
242,3\end{array}$ & 7 & $\begin{array}{r}600 \\
3300 \\
6000\end{array}$ & $\begin{array}{l}36,1 \\
61,6 \\
72,2\end{array}$ & $\begin{array}{l}48,6 \\
63,7 \\
67,7\end{array}$ & $\begin{array}{l}63,6 \\
73,4 \\
78,6\end{array}$ & $\begin{array}{l}148,3 \\
198,7 \\
218,5\end{array}$ \\
\hline & & 216,1 & 223,2 & 233,0 & 672,3 & $\mathrm{~S}$ & $\mathrm{~S}$ & 169,9 & 180,02 & 215,6 & 565,5 \\
\hline & $\begin{array}{r}600 \\
3300 \\
6000\end{array}$ & $\begin{array}{l}180,8 \\
216,8 \\
229,2\end{array}$ & $\begin{array}{l}204,3 \\
239,0 \\
234,5\end{array}$ & $\begin{array}{l}201,1 \\
244,8 \\
255,0\end{array}$ & $\begin{array}{l}586,2 \\
700,6 \\
718,7\end{array}$ & & $\begin{array}{lr}S & 600 \\
S & 3300 \\
S & 6000\end{array}$ & $\begin{array}{l}105,3 \\
185,6 \\
205,0\end{array}$ & $\begin{array}{l}148,0 \\
206,1 \\
210,6\end{array}$ & $\begin{array}{l}181,3 \\
233,8 \\
236,1\end{array}$ & $\begin{array}{l}434,6 \\
625,5 \\
651,7\end{array}$ \\
\hline & total & 626,8 & 677,8 & 700,9 & 2005,5 & & $S$ total & 495,9 & 564,7 & 651,2 & 1711,8 \\
\hline
\end{tabular}

Tabelle 4

Varianzanalyse der Werte von $\eta_{C}$ für die Summe der Sommer- und Winterexperimente $\left(\bar{t}=16,3^{\circ} \mathrm{C}\right.$, bzw. $\left.10,0^{\circ} \mathrm{C}\right)$

\begin{tabular}{lcrrrc}
\hline Variabilität & $\mathrm{Fg}$ & $\mathrm{SQ}$ & $\mathrm{DQ}$ & $\mathrm{F}$ & $\mathrm{P}$ \\
\hline Temperatur $t$ & 1 & $\mathbf{1 5 9 7 , 4 0}$ & 1597,40 & 80,6 & $\ll 0,001$ \\
$X_{1}^{\prime}$ & 2 & 4025,75 & 2012,87 & 101,6 & $\ll 0,001$ \\
$R_{h}$ & 2 & 1462,75 & 731,37 & 36,9 & $\ll 0,001$ \\
$\mathrm{O}^{\prime}$ & 2 & $\mathbf{7 9 , 6 4}$ & 39,82 & 2,01 & $>0,1$ \\
$t \cdot x_{1}^{\prime}$ & 2 & 242,13 & 121,06 & 6,11 & 0,005 \\
$t \cdot R_{h}$ & 2 & 202,40 & 101,20 & 5,1 & 0,01 \\
$t \cdot \mathrm{O}^{\prime}$ & 2 & 50,39 & 25,19 & 1,27 & $>0,1$ \\
Rest & 40 & 791,87 & 19,79 & & \\
Total & 53 & 8452,33 & - & & \\
\hline
\end{tabular}


4.11 Analyse des Gesamtversuches. Die Analyse ergibt folgendes Resultat: Die mittlere Temperaturdifferenz von rund $6^{\circ} \mathrm{C}$ zwischen den beiden Versuchsgruppen äussert sich in stark gesicherter Weise beim Reinigungseffekt der Anlagen. In gleicher Weise ist auch die Wirkung von hydraulischer Raumbelastung und Schlammkonzentration bei $\eta_{C}$ stark gesichert. Hingegen ist kein Einfluss der Sauerstoffspannung auf die Reinigung bezüglich der organischen C-Verbindungen innerhalb des untersuchten Bereiches von $\mathrm{I}-7 \mathrm{mg} \mathrm{O} / \mathrm{lim}$ Belüftungsbecken festzustellen.

Es bestehen stark gesicherte Wechselwirkungen zwischen der Temperatur einerseits und der Raumbelastung, bzw. der Schlammkonzentration anderseits, d.h. die beiden Parameter $R_{h}$ und $x_{1}$ wirken sich bei hoher, bzw. tiefer Temperatur in unterschiedlicher Weise auf den Reinigungseffekt aus. Eine Wechselwirkung Temperatur-Sauerstoffkonzentration ist nicht vorhanden.

4.12 Analyse der Sommer-und Winterversuche: Zur Präzisierung der Hauptwirkungen und der Wechselwirkungen der drei unabhängigen Variablen $R_{h}, x_{1}$ und $\mathrm{O}$ müssen die beiden Temperaturblöcke noch gesondert analysiert werden.

In beiden Temperaturbereichen ergeben sich prinzipiell gleiche Ergebnisse:

- Die Wirkung der Schlammkonzentration und der Raumbelastung ist sehr stark gesichert.

Tabelle 5

Varianzanalyse der Werte von $\eta$ der Sommer-und Wintermonate

\begin{tabular}{lrrrrl}
\hline Variabilität & Fg & SQ & DQ & F & P \\
\hline Sommer & & & & & \\
$x_{1}^{\prime}$ & 2 & 1147,08 & 573,54 & 28,3 & $\ll 0,001$ \\
$R_{h}$ & 2 & 319,46 & 159,73 & 7,88 & $<0,005$ \\
O & 2 & 123,05 & 61,52 & 3,04 & $<0,1$ \\
Rest*) & 20 & 404,88 & 20,24 & & \\
Total & 26 & 1994,47 & - & & \\
Winter & & & & & \\
$x_{1}$ & 2 & 3120,80 & 1560,40 & 80,6 & 0,001 \\
$R_{h}$ & 2 & 1345,69 & 672,84 & 34,7 & \\
O & 2 & 6,98 & 3,49 & 0,18 & \\
Rest*) & 20 & 386,99 & 19,34 & & \\
Total & 26 & 4860,46 & - & &
\end{tabular}

*) Wechselwirkungen innerhalb Temperaturblöcken nicht signifikant, deshalb ist ihr DQ mit der Reststreuung vereinigt. 
- Eine Wirkung der Sauerstoffspannung lässt sich in den Winterversuchen nicht nachweisen, in den Sommerversuchen ist sie aber gesichert $(P=$ $\mathrm{O}, \mathrm{I}$ ). Die Kontrolle der Einzelwerte zeigt, dass sich diese Sauerstoffwirkung auf den Bereich zwischen den Stufen I mg O/l bis $4 \mathrm{mg} \mathrm{O} / 1$ beschränkt.

- Wechselwirkungen zwischen den einzelnen Betriebsfaktoren lassen sich nicht nachweisen, d.h. die Wirkung der Schlammkonzentration ist bei allen geprïften Raumbelastungen und unabhängig davon gleichartig. Wenn man den Sauerstoffgehalt als Variable zunächst vernachlässigt, kann man die 3 Experimente mit gleichem Schlammgehalt und gleicher Raumbelastung, aber verschiedener Sauerstoffspannung für die Mittelwertbildung zusammenziehen, was eine erfreuliche Sicherung der Beobachtungen ergibt. Man findet dann folgende Mittelwerte des Reinigungseffektes bezüglich org. C:

Tabelle 6

Mittelwerte ${ }^{*}$ ) des Reinigungseffektes $\eta_{C} \%$

\begin{tabular}{|c|c|c|c|c|c|}
\hline$t$ & $x_{1}^{\prime}$ & $\begin{array}{l}R_{h} \\
58\end{array}$ & 29 & 9 & $\begin{array}{l}\text { Vertrauens- } \\
\text { grenzen, } \mathrm{P}=0,1\end{array}$ \\
\hline$>13^{\circ} \mathrm{C}$ & $\begin{array}{r}600 \\
3300 \\
6000\end{array}$ & $\begin{array}{l}60,3 \\
72,3 \\
76,4\end{array}$ & $\begin{array}{l}68,1 \\
79,7 \\
78,2\end{array}$ & $\begin{array}{l}67,0 \\
81,6 \\
85,0\end{array}$ & 4,2 \\
\hline$<11^{\circ} \mathrm{C}$ & $\begin{array}{r}600 \\
3300 \\
6000\end{array}$ & $\begin{array}{l}35,1 \\
61,9 \\
68,3\end{array}$ & $\begin{array}{l}49,3 \\
68,7 \\
70,2\end{array}$ & $\begin{array}{l}60,4 \\
77,9 \\
78,7\end{array}$ & 5,0 \\
\hline
\end{tabular}
*) Mittelwerte aus $\mathrm{N}=3 \times 3=9$ Versuchen mit je 9 Untersuchungstagen (total 81 Einzel-
beobachtungen).

Unter Berücksichtigung der Vertrauensgrenzen zeigt sich (vgl. auch Abb. I):

- Die Wirkungen der Schlammkonzentration und der hydraulischen Raumbelastung sind besonders bei niedrigen Temperaturen ausgeprägt, sie nehmen bei üblichen Sommertemperaturen des Abwassers stark ab;

- Die Schlammkonzentrationen 3300 und $6000 \mathrm{mg} / \mathrm{l}$ ergeben keine gesicherten Unterschiede bezüglich des Reinigungseffektes beim org. C.

- Bei höherer physiologischer Aktivität des Belebtschlamms im Sommer ist auch zwischen der Raumbelastung von rd. $29 \mathrm{~m}^{3} / \mathrm{m}^{3}$. Tag und von $\mathrm{rd}$. $9 \mathrm{~m}^{3} / \mathrm{m}^{3}$. Tag kein wesentlicher Unterschied bezüglich der Elimination von organischen $\mathrm{C}$-Verbindungen festzustellen.

Der gesicherte Einfluss der Sauerstoffkonzentration auf $\eta_{C}$ in den Sommerversuchen verlangt noch einen Hinweis: die Prüfung der Einzelergeb- 
org. C
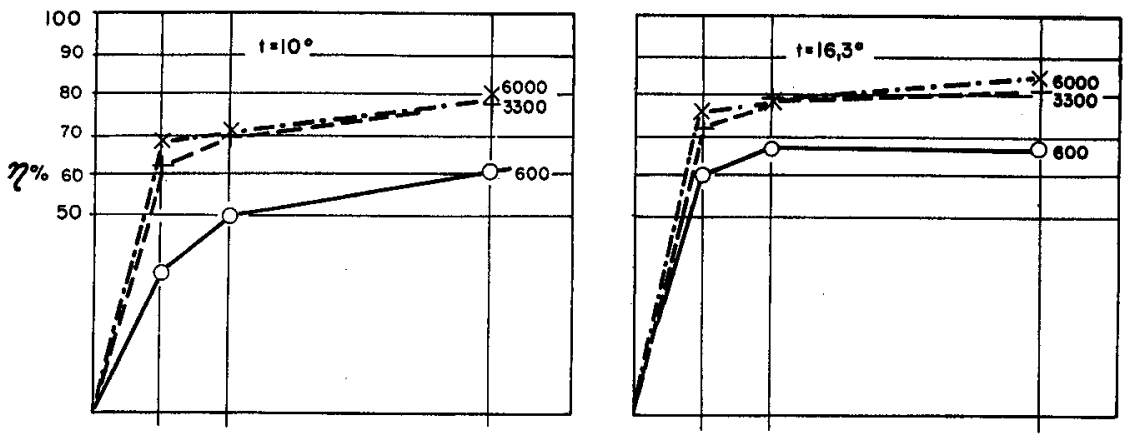

org. N
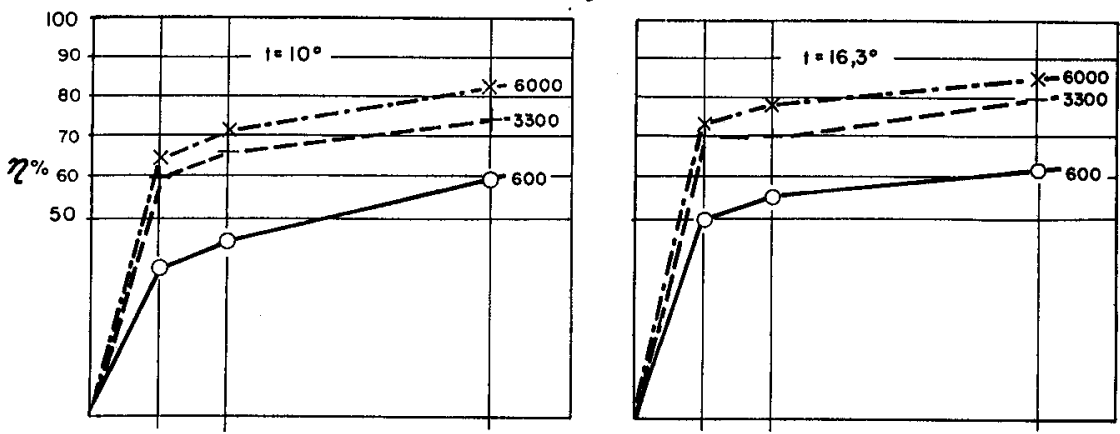

$\mathrm{BSB}_{5}$
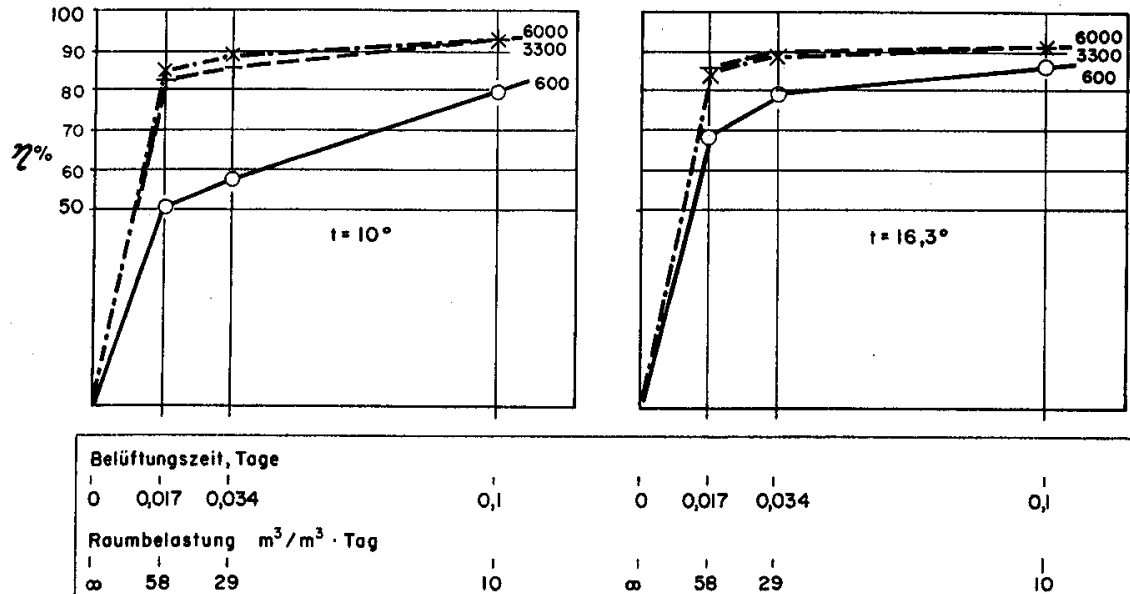

\section{Abbildung 1}

Reinigungseffekte bezüglich org. $\mathrm{C}$, org. $\mathrm{N}$ und $\mathrm{BSB}_{5}$ in Funktion der Belüftungszeit bei verschiedenen Schlammkonzentrationen im Belüftungsbecken und bei Sommer-, bzw. Wintertemperaturen. Jeder Punkt ist der Mittelwert aus drei Experimenten bei den O-Gehalten 1, 4 und $7 \mathrm{mg} / \mathrm{l} \mathrm{im}$ Belüftungsbecken. 
nisse zeigt, dass nur die Versuchsgruppen mit der Kombination $x_{3} / \mathrm{O}=$ $600 / \mathrm{r}$ und $x_{1} / \mathrm{O}=600 / 4$ gesichert voneinander abweichen (bei allen Raumbelastungen). Es handelt sich also um den Betrieb mit den höchsten Schlammbelastungen, bzw. mit den biologisch aktivsten Schlämmen (vgl. die endogene Atmung, WUHRMANN [20]), bei denen die Sauerstoffdiffusion ins Innere der Schlammflocken die kritische Sauerstoffspannung für die Atmung der einzelnen Zellen nicht mehr aufrechtzuerhalten vermag (WuHRMANN [I7, I9]).

\subsection{Elimination pon organischen Stickstoffperbindungen und $V$ erminderung des $B S B_{5}$}

Nach der absichtlich etwas ausführlich gestalteten Darstellung der Beurteilungsmethode unserer experimentellen Beobachtungen beim org. C können wir uns bei den beiden nächsten Masszahlen auf eine zusammenfassende Wiedèrgabe beschränken. Wie beim org. C ergibt sich auch beim $\mathrm{BSB}_{5}$ und den organischen $\mathrm{N}$-Verbindungen, dass von den vier unabhängigen Variablen nur drei, nämlich die Temperatur, die Raumbelastung und die Schlammkonzentration, einen statistisch gesicherten Einfluss auf den Reinigungseffekt ausüben. Wechselwirkungen zwischen den Faktoren sind ebenfalls nicht vorhanden (eine gutgesicherte Wechselwirkung $R_{h} x_{1}$ beim $\mathrm{BSB}_{5}$, Winterserie, ist auf die - in einigen Versuchen mit niederer Schlammkonzentration - aufgetretenen Trübungen im Anlagenabfluss zurückzuführen und hat im Rahmen dieser Fragestellung keine Bedeutung). Wir haben deshalb wiederum die Mittelwertsbildung der Reinigungseffekte über die drei Sauerstoffstufen vorgenommen und finden dann folgendes Ergebnis:

Tabelle 7

Mittelwerte *) des Reinigungseffektes $\eta_{N} \%$ bezüglich organischem Stickstoff

\begin{tabular}{|c|c|c|c|c|c|}
\hline$t$ & $x_{1}^{\prime}$ & $\begin{array}{l}R_{h} \\
58\end{array}$ & 29 & 9 & $\begin{array}{l}\text { Vertrauens- } \\
\text { grenzen, } P=0,1\end{array}$ \\
\hline$>13^{\circ} \mathrm{C}$ & $\begin{array}{r}600 \\
3300 \\
6000\end{array}$ & $\begin{array}{l}50,3 \\
71,2 \\
74,3\end{array}$ & $\begin{array}{l}55,7 \\
70,8 \\
78,8\end{array}$ & $\begin{array}{l}63,2 \\
80,6 \\
85,6\end{array}$ & 7,8 \\
\hline$<11^{\circ} \mathrm{C}$ & $\begin{array}{r}600 \\
3300 \\
6000\end{array}$ & $\begin{array}{l}36,8 \\
59,8 \\
65,0\end{array}$ & $\begin{array}{l}43,8 \\
66,4 \\
71,9\end{array}$ & $\begin{array}{l}60,1 \\
75,1 \\
83,2\end{array}$ & 9,4 \\
\hline
\end{tabular}

*) Mittelwerte aus $N=3 \times 3=9$ Versuchen (81 Einzelbeobachtungen)

Bei allen Tabellen sind wiederum die Vertrauensgrenzen für $\mathrm{P}=\mathrm{O}, \mathrm{I}$ angegeben. Wir möchten ausdrücklich darauf hinweisen, dass es sinnlos ist, 
Tabelle 8

Mittelwerte*) des Reinigungseffektes $\eta_{B} \%$ bezüglich $B S B_{5}$

\begin{tabular}{lrllll}
\hline$t$ & & $R_{h}$ & & & Vertrauens- \\
& $x_{1}^{\prime}$ & 58 & 29 & 9 & grenzen, P = 0,1 \\
\hline$>13^{\circ} \mathrm{C}$ & 600 & 68,4 & 79,3 & 85,9 & 5,5 \\
& 3300 & 86,3 & 90,1 & 91,1 & \\
$<11^{\circ} \mathrm{C}$ & 6000 & 85,4 & 88,7 & 91,4 & \\
& 600 & 50,3 & 57,1 & 79,3 & 4,4 \\
& 3300 & 82,3 & 84,9 & 92,7 & \\
& 6000 & 84,3 & 87,4 & 91,5 &
\end{tabular}

*) Mittelwerte aus $\mathrm{N}=3 \times 3=9$ Versuchen (81 Einzelbeobachtungen)

Mittelwerte in den vorstehenden Tabellen miteinander zu vergleichen, ohne ihre Variabilität und das Mass der Vertrauensgrenze zu berücksichtigen.

Aus der Zusammenfassung in Abb. I ist sofort ersichtlich, dass analog wie beim org. C die Steigerung der Schlammkonzentration von 3300 auf $6000 \mathrm{mg} / \mathrm{lim}$ Belüftungsbecken keinen ins Gewicht fallenden Vorteil für die Verminderung des $\mathrm{BSB}_{5}$ oder org. $\mathrm{N}$ mit sich brachte. Es ist aber zu berücksichtigen, dass diese Feststellung nicht allgemeinguiltigen Charakter besitzt, sondern ausschliesslich für ein verdünntes Rohabwasser gilt wie im Falle unserer Versuche. Bei wesentlich grösseren Abwasserkonzentrationen würde sich diese Grenze der unkritischen Schlammkonzentration (etwa $3300 \mathrm{mg} / \mathrm{l}$ ) naturgemäss nach höheren Werten verschieben.

\section{5. Überschußschlammproduktion}

Die genaue Feststellung der Überschußschlammproduktion in einer Belebtschlammanlage - auch wenn sie nur geringe Dimensionen besitzt - ist nicht einfach. Die meisten veröffentlichten Zahlen dürften deshalb mehrheitlich nur Näherungswerte darstellen. Auch in unseren Versuchen erwiesen sich die Streuungen der Produktionswerte als so gross, dass eindeutige Aussagen über die Wirkung einzelner Betriebsparameter auf die Schlammproduktion nur nach sorgfältiger statistischer Analyse der Beobachtungen gemacht werden können.

Grundsätzlich besteht der Überschußschlamm aus den suspendierten Stoffen SS, welche mit dem gereinigten Abwasser abfliessen (Schwebestoffe im nachgeklärten Abfluss) und den Feststoffen ƯS, die mit dem eigentlichen Überschußschlamm separat aus dem System entfernt werden. Für den Praktiker spielt allerdings nur die letztere Komponente eine Rolle; für jeg- 
liche Betrachtung über die Abhängigkeit der Schlammproduktion von den Betriebsbedingungen muss hingegen die gesamte abfliessende Feststoffmenge berücksichtigt werden (vgl. Abschnitt 2). Die Verteilung auf die beiden Komponenten - Schwebestoffe im Abfluss und Schlammablass - ist ja nur zufälliger Natur, bzw. nur von den technischen Bedingungen der Nachklärung abhängig.

\subsection{Absolute Überscbußscblammengen bei perscbiedenen Betriebszuständen}

Wir stellen zunächst in der Tab. 9 die bei unseren Versuchen beobachtete Feststoffproduktion pro $\mathrm{m}^{3}$ verarbeitetes $A$ bwasser als Trockenstoffe und als org. C zusammen. Die Auswertung aller Versuche ergibt, dass in Úbereinstimmung mit der theoretischen Forderung (Gl. I3) weder ein signifikanter Einfluss der hydraulischen Raumbelastung (d.h. der Belüftungszeit) noch der Schlammkonzentration im Belüftungsbecken auf die Schlammproduktion besteht. Hingegen ist ein deutlicher Hinweis (nicht in allen Fällen bei $\mathrm{P}=0$, I gesichert) auf eine Verminderung der Überschußschlammenge mit zunehmender O-Spannung im Beliuftungsbecken erkennbar. Die Feststoffproduktion in den beiden untersuchten Temperaturbercichen unterscheidet sich in statistisch knapp gesicherter Weise, indem bei den tiefen Temperaturen etwa 10\% mehr Feststoffe anfallen als bei Sommertemperaturen.

Die absoluten Produktionswerte von Tab. 9 gelten nur für relativ dünnes Rohwasser. Eine Beziehung allgemeinerer Art, in welcher die Abwasser-

Tabelle 9

Uberschußschlammproduktion: $\mathrm{g}$ Trockensubstanz $/ \mathrm{m}^{3}$ Rohwasser und $\mathrm{g}$ org. $\mathrm{C} / \mathrm{m}^{3}$ Rohwasser bei $R_{h}=9$ bis $58 \mathrm{~m}^{3} / \mathrm{m}^{3}$. Tag und $x_{1}^{\prime}=600$ bis $6000 \mathrm{mg} / 1^{1}$ )

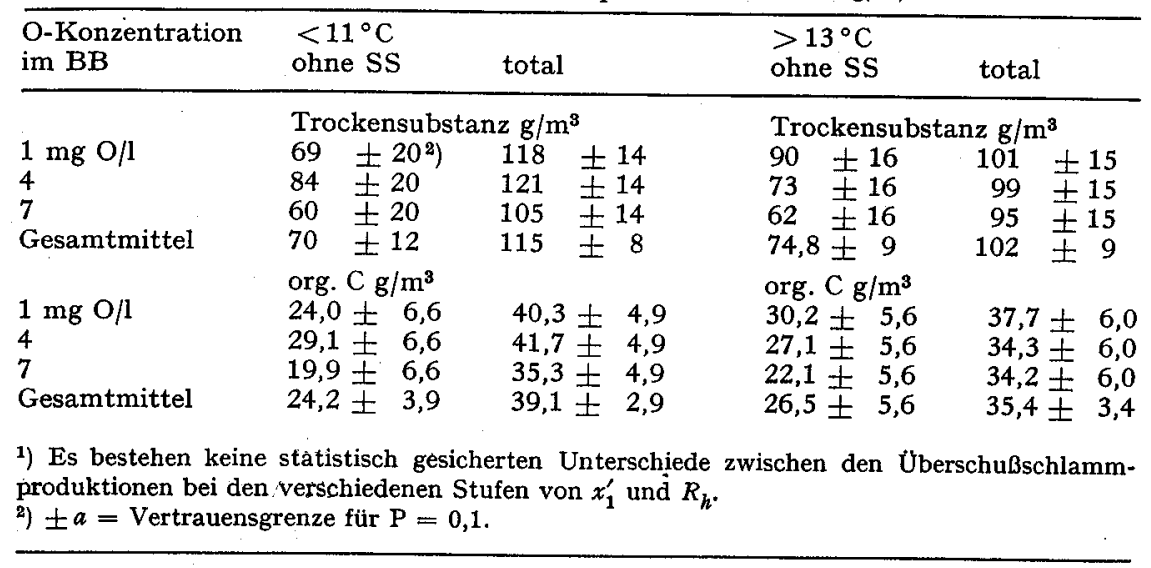


konzentration zum Ausdruck käme, lässt sich aus diesen Werten nicht ableiten. Hingegen können sie zur Berechnung des "Ausnützungskoeffizienten» $Y_{S}$ gemäss Gl. (I3) verwendet werden (vgl. Abschnitt 5.3).

\subsection{Zusammensetzung der Überschußscblämme}

Die Ưberschußschlämme wurden bei allen Versuchen regelmässig auf ihren Gehalt an organischen Stoffen (Glühverlust,) org. Kohlenstoff, org. Stickstoff, Gesamtphosphor und Kalium untersucht. Die Mittelwerte und Standardabweichungen der Analysen aus allen Experimenten sind in Tab. Io getrennt für die Winter -und Sommerperioden zusammengefasst.

Tabelle 10

Zusammensetzung des Überschußschlamms, Gesamtmittel*) der Temperaturblöcke

\begin{tabular}{|c|c|c|c|c|}
\hline & & $<11^{\circ} \mathrm{C}$ & $>13^{\circ} \mathrm{C}$ & $\begin{array}{l}\text { Differenz zwischen } \\
\text { Temperaturblöcken } \\
(\mathrm{P}=0,1)\end{array}$ \\
\hline Asche & $\%$ & 36 & 32,76 & \\
\hline Glühverlust & $\%$ & $64,00 \pm 2,72$ & $67,24 \pm 2,72$ & sehr stark gesichert \\
\hline org. C & $\%$ & $33,98 \pm 1,84$ & $34,43 \pm 1,84$ & nicht gesichert \\
\hline Gesamt-N & $\%$ & $4,97 \pm 0,96$ & $5,78 \pm 0,96$ & sehr stark gesichert \\
\hline Gesamt-P & $\%$ & $1,27 \pm 0,52$ & $1,48 \pm 0,52$ & nicht gesichert \\
\hline Gesamt-K & $\%$ & $0,40 \pm 0,16$ & $0,43 \pm 0,16$ & nicht gesichert \\
\hline \multicolumn{5}{|c|}{$\begin{array}{l} \pm s=\text { Standardabweichung aller Mittelwerte der } 54 \text { Versuche. } \\
\text { *) Mit Ausnahme des Gesamt-N besteht keine gesicherte Abhängigkeit }(P<0,1) \text { zwischen Be- } \\
\text { triebsbedingungen und Schlammzusammensetzung innerhalb der Temperaturblöcke. }\end{array}$} \\
\hline
\end{tabular}

Man findet einen kleinen, aber statistisch sehr gut gesicherten Unterschied bezüglich des Glühverlustes und des Gehaltes an org. $\mathrm{N}$ zwischen den Schlämmen aus der Winter- bzw. Sommerperiode. Eine entsprechende Differenz im Gehalt an org. C ist andeutungsweise vorhanden, statistisch aber nicht gesichert. Im P- und K-Gehalt liegen keine Unterschiede vor, die unter Beruicksichtigung der Streuungen signifikant wären.

Beim Gehalt an organischem Stickstoff lässt sich eine deutliche Abhängigkeit der Schlammzusammensetzung von der Belebtschlammkonzentration im Belüftungsbecken erkennen. Die Streuungszerlegung in Tab. II a und die Durchschnittswerte in Tab. IIb enthalten die entsprechenden Belege.

Dank dieser Abhängigkeit des org. N-Gehaltes von den Betriebsbedingungen ist es möglich, einen weiteren wichtigen Einblick in den Mechanismus der Überschußschlammproduktion zu gewinnen, wie weiter unten zu zeigen sein wird. 
Tabelle 11

Abhängigkeit des Gehaltes an org. $N$ im Überschußschlamm von den Betriebsfaktaren

a) Varianzanalyse

\begin{tabular}{lrrrrl}
\hline Variabilität & Fg & SQ & DQ & F & P \\
\hline Temperatur & 1 & 7,2399 & 7,2399 & 20,9 & 0,001 \\
$R_{h}$ & 2 & 1,3779 & 0,6889 & 0,19 & 0,3 \\
$x_{1}$ & 2 & 25,7704 & 12,8852 & 37,2 & 0,001 \\
$O$ & 2 & 0,3202 & 0,1601 & 0,046 & 0,3 \\
Rest & 38 & 13,1603 & 0,3463 & & \\
Insgesamt & 45 & 47,8687 & - & & \\
\end{tabular}

b) Mittelwerte des \%-Gehaltes an org. N für die Raumbelastungen 9 bis $58 \mathrm{~m}^{3} / \mathrm{m}^{3} \cdot$ Tag und $O-G$ ehalte im Belüftungsbecken von 1 bis $7 \mathrm{mg} / 1$

\begin{tabular}{rll}
\hline \multicolumn{1}{c}{$x_{1}^{\prime}$} & $<11^{\circ} \mathrm{C}$ & $>13^{\circ} \mathrm{C}$ \\
\hline 600 & $3,66 \%$ & $4,91 \%$ \\
3300 & $5,22 \%$ & $6,17 \%$ \\
6000 & $6,14 \%$ & $6,26 \%$
\end{tabular}

Vertrauensgrenzen der Mittelwerte für $\mathrm{P}=0,1: \pm 0,22 \%$.

\subsection{Beziehung zmischen der Überschußscblammproduktion und den Betriebsparametern}

Die im System gebildete und zur Beibehaltung eines Beharrungszustandes der Schlammkonzentration notwendigerweise zu entfernende Feststoffmenge ergibt sich schematisch aus den in Abb. 2 dargestellten Beziehungen.

Org. C

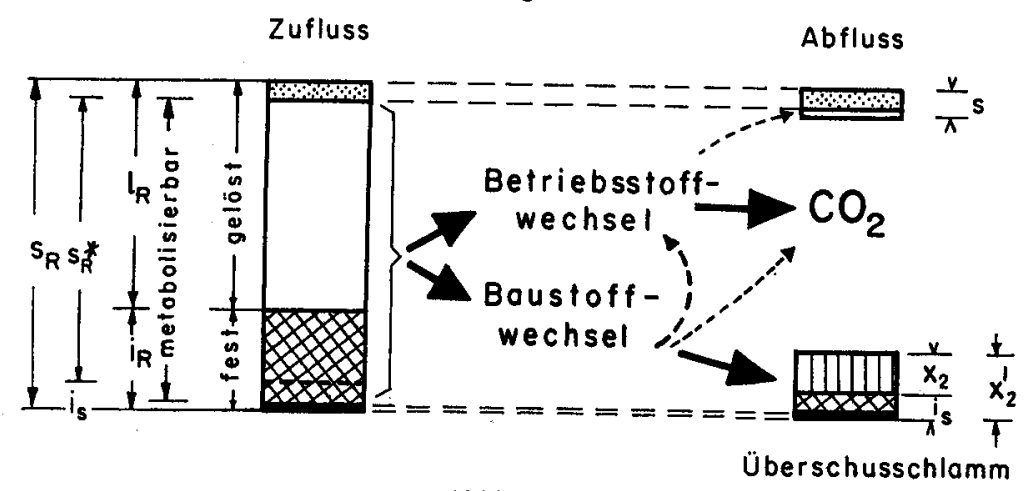

Abbildung 2 
Im Beharrungszustand des Betriebes müssen vor allem einmal jene Mengen von Schwebestoffen $i_{s}$ des Rohwassers, die biologisch nicht angegriffen wurden (inertes und langsam abbaubares Material), wieder aus dem System entlassen werden (wir setzen voraus, dass sie quantitativ ausflocken). Die festen und gelösten Inhaltsstoffe des Rohwassers, welche metabolisiert werden, gehen zum Teil in den Betriebsstoffwechsel und zum Teil in den Baustoffwechsel der Schlammorganismen ein. Die Aufteilung des Gesamtsubstratverbrauchs in diese beiden Stoffwechselwege bestimmt die Menge der neugebildeten Feststoffe. Sodann kann je nach Betriebsbedingungen ein mehr oder weniger grosser Teil des im Baustoffwechsel assimilierten Substrats wieder in den Betriebsstoffwechsel übergehen (transitorische Reservestoffe, Substrate der endogenen Atmung). Wenn man das vorstehende Schema betrachtet, ergibt sich sofort, dass das einzige Mass für die Úberschußschlammproduktion, das direkt mit dem Stoffwechsel des Systems verbunden ist, der sog. "Ausnützungsfaktor» $Y_{S}$ gemäss Gl. (I3) darstellt. Er ist dort definiert als Gewicht der gebildeten Organismenmasse pro Gewichtseinheit verbrauchten Substrats oder

$$
\begin{gathered}
x_{2}=Y_{S}\left(s_{R}^{*}-s^{*}\right), \\
\left(s_{R}^{*}=\text { metabolisierbarer Anteil der org. C-Verbindungen }\right) .
\end{gathered}
$$

Als gemeinsame Masseinbeit für die Schlammenge und den Substratperbrauch stebt uns ausscbliesslich der organische Koblenstoff zur Verfügung.

Der Wert von $Y_{S}$ ist ein objektives Mass für die Beeinflussung der Schlammproduktion durch die verschiedenen Betriebsbedingungen, und $z w a r$ unabhängig von der absoluten Substratkonzentration. $Y_{S}$ ist allerdings von der Qualität des Substrats abhängig, doch dürfte innerhalb der Gruppe "städtisches Abwasser ohne spezielle industrielle Belastungen" dieser Faktor nur eine untergeordnete Rolle spielen. Da man bei der Analyse eines Anlagenzuflusses die nicht abbaubaren und die abbaubaren Stoffe gemeinsam erfasst, ist im Gesamtabbau $\left(s_{R}-s\right)$ auch der auf rein physikalischem Wege in den Schlamm übergeführte Schmutzstoffanteil des Rohwassers (soweit er als org. C-Verbindungen vorliegt) enthalten. Der "Ausnützungskoeffizient" $Y_{s}^{\prime}$, der aus der Konzentrationsabnahme des gesamten org. Cerrechnet wird, ist deshalb nur ein Scheinwert, welcher zum Beispiel stark vom inerten Anteil der Feststoffe des Rohwassers beeinflusst ist. Wir schreiben die Beziehung (I3) für die Bruttoüberschußschlammproduktion (inklusive nichtabgebaute primäre Schwebestoffe) deshalb besser mit

$$
x_{2}^{\prime}=Y_{S}^{\prime}\left(s_{R}-s\right) \text {. }
$$


Weiter unten werden wir eine Berechnung des "echten" biologischen Ausnützungskoeffizienten $Y_{S}$ versuchen, da dieser Wert z. B. für die Abschätzung des Sauerstoffverbrauchs im Belüftungsbecken eine kausale Grösse darstellt.

Die Varianzanalyse aller Werte von $Y_{S}^{\prime}$ aus den beiden Temperaturserien ergibt, dass von den vier abhängigen Variablen nur die Temperatur (schwach gesichert), die Schlammkonzentration und die Sauerstoff konzentration (beide sehr gut gesichert) von Einfluss sind. Wir geben deshalb in Tab. I2 die gefundenen Werte von $Y_{S}^{\prime}$ als Durchschnitt uiber alle 3 untersuchten Raumbelastungen.

Tabelle 12 Durchschnitte von $Y_{S}^{\prime}$ ( $g$ org. C im Gesamtfeststoffabfluss/g org. C-Elimination) über alle
Beliuftungszeiten

\begin{tabular}{|c|c|c|c|c|c|c|}
\hline $\begin{array}{l}\text { Temp. } \\
\text { O mg/1 } \\
x_{1}^{\prime} \mathrm{mg} / 1\end{array}$ & ${ }_{1}^{<11^{\circ} \mathrm{C}}$ & 4 & 7 & $>_{1}^{>13}$ & 4 & 7 \\
\hline $\begin{array}{r}600 \\
3300 \\
6000\end{array}$ & $\begin{array}{l}\left.0,86^{1}\right) \\
0,82 \\
0,64\end{array}$ & $\begin{array}{l}0,76 \\
0,80 \\
0,66\end{array}$ & $\begin{array}{l}0,77 \\
0,77 \\
0,51\end{array}$ & $\begin{array}{l}0,79 \\
0,77 \\
0,75\end{array}$ & $\begin{array}{l}0,69 \\
0,67 \\
0,52\end{array}$ & $\begin{array}{l}0,68 \\
0,57 \\
0,44\end{array}$ \\
\hline Mittel & \multicolumn{3}{|c|}{$\left.0,73^{2}\right)$} & \multicolumn{3}{|c|}{0,66} \\
\hline \multicolumn{7}{|c|}{$\begin{array}{l}\text { 1) Vertrauensgrenze für } P=0,1 \text { bei Einzelwerten }= \pm 0,068 \\
\text { 2) Vertrauensgrenze für } P=0,1 \text { bei Mittelwerten }= \pm 0,058\end{array}$} \\
\hline
\end{tabular}

Die Werte von $Y_{s}^{\prime}$ in Tab. I2 sind berechnet auf der Basis des gesamten Feststoffabflusses aus den Anlagen (Schwebestoffe im Nachklärbeckenabfluss + Feststoffe des Überschußschlamms). Innerhalb des untersuchten Belastungsbereiches kann man einen durchschnittlichen Annäherungswert für den Anteil der Schwebestoffe im Abfluss des Nachklärbeckens am Gesamtfeststoffabfluss von rd. $15 \%$ angeben (stark von den Betriebsbedingungen abhängig!). Will man deshalb die Fraktion $Y_{S}^{\prime}$ ausschliesslich auf den eigentlichen Überschußschlamm beziehen, so muss man die Ziffern in Tab. I2 um I2 $\%$ vermindern.

Anhand der Vertrauensgrenzen von $\mathrm{P}=0, \mathrm{x}$ ergibt sich (übereinstimmend mit Tab. 9) sofort, dass die Bruttoschlammproduktion $x_{2}^{\prime}$, gemessen am gesamten Substrat "verbrauch" (org. C), im Winter etwa 1o\% höher ist als im Sommer. Ferner nimmt allgemein die Produktionshöhe mit zunehmendem Schlammgehalt und zunehmendem O-Gehalt im Belüftungsbecken deutlich ab (grösste Variation für $Y_{s}^{\prime}:$ von $600 / \mathrm{I}$ zu $6000 / 7=0,86 \rightarrow 0,5 \mathrm{I}$, bzw. 0,79 $\rightarrow 0,44$ ). Die Abhängigkeit der absoluten ÚS-Mengen von der 
Schlammkonzentration, welche in Tab. 9 von der Variabilität der Rohwasserkonzentration verdeckt wurde, kommt bei der Relativzahl $Y_{S}^{\prime}$ deutlich zum Ausdruck.

Die Grössenunterschiede von $Y_{S}^{\prime}$ gemäss Tab. I2 stehen im Widerspruch zur theoretischen Forderung gemäss Gl. (13), wonach die Überschußschlammproduktion lediglich von der Menge verbrauchten Substrats und nicht von den Betriebsbedingungen einer Anlage abhängig sein sollte. Die beobachtete Veränderung von $Y_{S}^{\prime}$ in Funktion des Schlammgehalts und der Sauerstoffspannung im Belüftungsbecken weist also auf zusätzliche Faktoren hin, welche unter den speziellen Bedingungen eines Belebstchlammsystems die ƯS-Menge bestimmen. Wir haben bereits einleitend angedeutet, dass sich die Autolyse des Schlamms und weitere Faktoren (z.B. Frass durch Ziliaten) im Sinne einer Verminderung der ÜS-Menge bemerkbar machen müssen, und anderseits die unzersetzlichen Schwebestoffe des Abwassers zu seiner Vermehrung beitragen. Die Bruttoproduktionsformel (I5) muss deshalb wie folgt in Einzelsummen zerlegt werden:

$$
\begin{array}{llll}
\begin{array}{l}
\text { Bruttoüberschuß- } \\
\text { schlammenge }
\end{array} & \begin{array}{l}
\text { Organismen- } \\
\text { zuwachs }
\end{array} & \begin{array}{c}
\text { unzersetzte } \\
\text { primäre } \\
\text { Schwebestoffe }
\end{array} & \begin{array}{c}
\text { Verluste } \\
\text { durch Auto- } \\
\text { lyse usw. }
\end{array} \\
x_{2}^{\prime}=Y_{S}^{\prime}\left(s_{R}-s\right)=Y_{S}\left(s_{R}^{*}-s^{*}\right) & +\left(i_{R}-i_{s}\right) & -\lambda x_{1}^{\prime} \cdot \quad
\end{array}
$$

Wir besitzen heute keine Unterlagen, um die einzelnen Summanden in vorstehender Formel beziffern zu können. Die Verminderung des Bruttoausnützungskoeffizienten $Y_{S}^{\prime}$ mit zunehmender Temperatur, Schlammkonzentration und Sauerstoff konzentration im Belüftungsbecken gemäss Tab. I2 deutet nur an, dass unter diesen Einflüssen der Anteil unzersetzter primärer Schwebestoffe im Überschußschlamm abnimmt und die Verluste sich verstärken. Es ist deshalb eine zwingende Schlussfolgerung - und sie wird durch die Beobachtungen bei den sog. Volloxydationsanlagen bestätigt -, dass man unter geeigneten Betriebsbedingungen die Überschußschlammenge sehr stark vermindern kann. Dem "Verlustkoeffizienten" $\lambda$ kommt dabei offensichtlich eine besonders grosse Bedeutung $z u$, da ja die Menge primärer Schwebestoffe (mit oder ohne Vorklärung) bei einem häuslichen Abwasser grössenordnungsmässig konstant ist. Leider ist es nicht möglich, mit den vorliegenden Versuchsdaten die absolute Grösse von $\lambda$ in Funktion der Betriebsbedingungen zu berechnen. Wir werden in einer späteren Arbeit anhand zusätzlicher Beobachtungen auf dieses Problem zurückkommen. - Hingegen kann auf Grund der vorliegenden Messungen wenigstens die Frage des Úbergangs primärer Schwebestoffe (aus einem 
vorgeklärten Abwasser) in den Überschußschlamm quantitativ diskutiert werden (vgl. Abschnitt 5.5).

Für den Praktiker sind die in Tab. I2 vereinigten Werte für den Bruttoüberschußschlammkoeffizienten $Y_{S}^{\prime}$ im Rahmen üblicher Betriebsbedingungen von Belebtschlammanlagen für städtisches Abwasser (unabhängig von der Konzentration!) eine ausreichende Berechnungsgrundlage für den Überschußschlammanfall. In Anbetracht der Streuungen ist es ausreichend, mit den Mittelwerten für $Y_{S}^{\prime}$ innerhalb des von uns untersuchten Bereiches von Betriebsbedingungen zu rechnen. Man erhält also folgende Berechnungsformeln:

Úberschußschlammenge bei Sommertemperaturen:

$$
R_{h} x_{2}^{\prime}=0,66 R_{h}\left(s_{R}-s\right),
$$

bei Wintertemperaturen:

$$
\left.R_{h} x_{2}^{\prime}=0,73 R_{h}\left(s_{R}-s\right) \quad \text { [kg org. C } / \mathrm{m}^{3}{ }_{B B} \cdot \mathrm{Tag}\right] .
$$

Da im Mittel der Überschußschlamm $34,4 \%$ org. C enthielt, kann man eine Umrechnung in Trockenstoffe leicht vornehmen.

Approximatipe Berechnung der Überschußschlammproduktion auf Basis des $B S B_{5}$ : Man kann eine grobe Annäherungsberechnung für die ÜS-Produktion auf der Basis der $\mathrm{BSB}_{5}-\mathrm{Abnahme}$ vornehmen, wenn man eine Beziehung (die selbstverständlich rein empirischer Art ist) zwischen dem Gehalt des Abwassers an organischem Kohlenstoff und seinem $\mathrm{BSB}_{5}$ herstellt. Wir haben bei allen Versuchen sowohl für das vorgeklärte Abwasser als auch für die biologisch gereinigten Abflüsse die Proportion $\mathrm{BSB}_{5} /$ org. $\mathrm{C}$ gebildet und fanden folgende Werte:

$$
\begin{aligned}
& \text { vorgeklärtes Abwasser } \quad \mathrm{BSB}_{5}=\text { etwa } \mathrm{I}, 9 \cdot \text { org. } \mathrm{C}, \\
& \text { Nachklärbeckenabfluss } \\
& \mathrm{BSB}_{5}=\text { etwa } \mathrm{I} \cdot \text { org. C. }
\end{aligned}
$$

Will man die vorstehenden Berechnungsformeln mit $\mathrm{BSB}_{5}$-Werten benützen, so muss man den Ausnützungskoeffizienten $Y_{S}^{\prime}$ in die - allerdings unsinnige -, Dimension: $g$ gebildete Trockensubstanz $/ \mathrm{g} \mathrm{BSB} \mathrm{B}_{5}-\mathrm{Abnahme}$ bringen. Für den Zähler gilt dieselbe Umrechnung wie für $x_{2}^{\prime}(34,4 \%$ org. $C$ in den Trockenstoffen). Für die $\mathrm{BSB}_{5}-\mathrm{Abnahme}$ (Nenner) kann man beispielsweise einen Kohlenstoffwert von 1,45 (Mittelwert von $I, 9$ und $\mathrm{I}, 0$ ) wählen. Mit Hilfe dieser Umrechnungskünste gelangt man dann zu

$$
x_{2}^{\prime}=\frac{0,66}{\mathrm{I}, 45}\left(\mathrm{I}, 9 s_{R}-s\right)=0,85 s_{R}-0,45 s \quad\left[\mathrm{~g} \mathrm{TS} / \mathrm{m}^{3} \mathrm{RW}\right],
$$


wobei $s_{R}$ und $s$ als $\mathrm{g} \mathrm{BSB}_{5} / \mathrm{m}^{3}$ einzusetzen sind. Da die Berechnung von $x_{2}^{\prime}$ grundsätzlich auch die Trockenstoffabgänge in Form der Schwebestoffe im Nachklärbeckenabfluss einschliesst, ist diese Trockenstoffmenge für die Berechnung des eigentlichen Überschußschlamms in Abzug zu bringen. Je nach Anlage und Betriebsweise kann diese Grösse in weiten Grenzen schwanken. Setzt man einen durchschnittlichen Wert von $20 \mathrm{mg} / \mathrm{l}$ an (entspricht einem ziemlich klaren Abfluss ohne auffälliges Flockentreiben, aber noch nicht einem "brillanten" Abfluss), so gelangt man zu folgender Approximationsberechnung:

$$
x_{2}^{\prime}=0,85 s_{R}-0,45 s-20 \quad\left[\mathrm{~g} T S / \mathrm{m}^{3} \mathrm{RW}\right] .
$$

Wir haben zur Kontrolle bei allen 27 Sommerversuchen diese "Berechnung" durchgeführt und finden als Gesamtmittel einen Wert von $x_{2}^{\prime}=70 \mathrm{~g}$ $\mathrm{TS} / \mathrm{m}^{3}$. Die effektiv gemessene Überschußschlammenge beträgt im Mittel aller Versuche $74,8 \mathrm{~g}$ TS $/ \mathrm{m}^{3}$, so dass man von einer brauchbaren Übereinstimmung dieser Mittelwerte reden kann. Es soll aber nicht verschwiegen werden, dass die für jeden Versuch berechneten Einzelwerte oft erheblich von den Messungen abwichen, wobei die fehlende Übereinstimmung zur Hauptsache auf die Korrektur mit dem schematischen Wert von $20 \mathrm{mg} / \mathrm{l}$ für die Schwebestoffe im Abfluss zurückzuführen war.

\subsection{Abbängigkeit der Überscbußschlammproduktion pon der $B S B_{5}$-Belastung.}

Man kann die gebildete Überschußschlammenge auch in Funktion der $\mathrm{BSB}_{5}$-Belastung einer Anlage ausdrücken, wie dies DownING et al. [2] versucht haben. Gemäss den Ausführungen in Abschnitt 2 ist diese Beziehung zwar ohne theoretische Begründung, da die Überschußschlammenge grundsätzlich nur durch die Menge eliminierten Substrats (und im Falle von Abwässern durch die zusätzliche Menge der durch die Anlage geschleppten, unzersetzlichen primären Schwebestoffe) bestimmt wird. Bildet man den Quotienten $x_{2}^{\prime} / s_{R}\left(s_{R}\right.$ als $\mathrm{BSB}_{5}$ gerechnet) bei unseren 54 Versuchen, so findet man erwartungsgemäss auf Grund der Varianzanalyse für $Y_{S}^{\prime}$, dass auch für diesen neuen Quotienten keine Abhängigkeit von der Belüftungszeit und der Schlammkonzentration besteht, hingegen eine messbare Wirkung der Temperatur und der Sauerstoffspannung im Belüftungsbecken auftritt. Die über Schlammkonzentration und Belüftungszeit gemittelten Werte für $x_{2}^{\prime} / s_{R}$ sind in Tab. I3 zusammengestellt.

Die Zahlen für die totale Feststoffproduktion (inkl. Schwebestoffe des Abflusses) fügen sich einigermassen in die graphische Darstellung ein, die DowNING für diesen Quotienten in Funktion der Belüftungszeit aufge- 
Tabelle 13

UtS-Produktion kg TS/kg BSB $B_{5}-Z$ ufluss bei $R_{h}=9$ bis $58 \mathrm{~m}^{3} / \mathrm{m}^{3} \cdot$ Tag und Schlammkonzentrationen von $x_{1}^{\prime}=600$ bis $6000 \mathrm{mg} / \mathrm{l}$

\begin{tabular}{|c|c|c|c|c|}
\hline $\begin{array}{l}\text { O-Konzentration } \\
\text { im BB }\end{array}$ & $\begin{array}{l}<11^{\circ} \mathrm{C} \\
\text { ohne SS }\end{array}$ & total & $\begin{array}{l}>13^{\circ} \mathrm{C} \\
\text { ohne SS }\end{array}$ & total \\
\hline & \multicolumn{2}{|c|}{ Trockensubstanz } & \multicolumn{2}{|c|}{ Trockensubstanz } \\
\hline $\begin{array}{l}1 \mathrm{mg} \mathrm{O} / 1 \\
4 \\
7\end{array}$ & $\begin{array}{l}\left.0,64 \pm 0,16^{*}\right) \\
0,69 \pm 0,16 \\
0,52 \pm 0,16\end{array}$ & $\begin{array}{l}1,09 \pm 0,13 \\
1,03 \pm 0,13 \\
0,88 \pm 0,13\end{array}$ & $\begin{array}{l}0,88 \pm 0,15 \\
0,64 \pm 0,15 \\
0,52 \pm 0,15\end{array}$ & $\begin{array}{l}1,11 \pm 0,17 \\
0,86 \pm 0,17 \\
0,82 \pm 0,17\end{array}$ \\
\hline Gesamtmittel & $0,62 \pm 0,10$ & $1,00 \pm 0,07$ & $0,68 \pm 0,09$ & $0,93 \pm 0,10$ \\
\hline
\end{tabular}

zeichnet hat. Die Kurve von DownING deutet an, dass bei Belüftungszeiten von mehrals $\mathrm{rd} .2-2,5 \mathrm{Std}$. $\left(R_{h} \leqslant \mathrm{I} 2 \mathrm{~m}^{3} / \mathrm{m}^{3}\right.$. Tag) die ÜS-Menge pro $\mathrm{kg}$ zugeführten $\mathrm{BSB}_{5}$ abzunehmen beginnt. Unsere Beobachtungen sind eine indirekte Bestätigung dieser Angabe, denn wir haben in dem von uns untersuchten Bereich von Belüftungszeiten ( 0,4 bis 2,5 Std.) in der Tat noch keine Abhängigkeit der Überschußschlammenge von der Belüftungszeit gefunden. Wir möchten aber erneut darauf hinweisen, dass die von DownING verwendete Korrelation keine theoretische Basis besitzt und deshalb von fragwürdiger Aussagekraft ist.

\subsection{Anteil pon Feststoffen des Robwassers im Überscbußscblamm der biologischen Stufe}

Es ist zurzeit nicht genauer bekannt, wieweit die suspendierten Stoffe, die mit dem vorgeklärten Abwasser in eine Belebtschlammstufe gelangen ("primäre Schwebestoffe »), zersetzt werden, bzw. welcher prozentuale Anteil dieser Feststoffe im Überschußschlamm noch enthalten ist. Die in Tab. II nachgewiesene Variabilität des N-Gehaltes der Schlämme lässt darauf schliessen, dass je nach Betriebsbedingungen dieser Anteil wechselt. Eine direkte Messung ist allerdings nicht möglich, doch kann eine rechnerische Abschätzung vorgenommen werden, wenn man von den Hauptkomponenten des Schlamms, dem organischen $\mathrm{C}$ und organischen $\mathrm{N}$, ausgeht.

Aus Abb. 2 ergibt sich:

$$
\begin{aligned}
& s_{R}=i_{R}+l_{R}, \\
& s_{R}^{*}=i_{R}-i_{s}+l_{R}-s_{i}=s_{R}-i_{s}-s_{i} .
\end{aligned}
$$

Da im Nachklärbecken keine Schlammveränderung eintritt, ist die Gesamtüberschußschlammenge

$$
x_{2}^{\prime}=x_{2}+i_{s} \text {. }
$$


Nach der früheren Formel (I3) ist der Organismenzuwachs, der ja nur aus angreifbaren Substraten erfolgen kann,

bzw. aus (I6) und (I7):

$$
x_{2}=Y_{S}\left(s_{R}^{*}-s^{*}\right) \text {, }
$$

$$
Y_{S}=\frac{x_{2}^{\prime}-i_{s}}{\left(s_{R}-s\right)-i_{s}}
$$

$Y_{S}$ stellt dabei den "echten" Ausnützungsfaktor dar, im Gegensatz zum "Brutto »ausnützungsfaktor $Y_{S}^{\prime}$, der aus $x_{2}^{\prime}$ und $\left(s_{R}-s\right)$ nach $(\mathbf{I} 5)$ berechnet wurde.

Es muss ferner die Bilanzbeziehung bezüglich N-Gehalt der Feststoffe erfüllt sein:

bzw.

$$
\begin{gathered}
x_{2}^{\prime} N_{x^{\prime}}=x_{2} N_{x}+i_{s} N_{i_{s}}, \\
i_{s}=\frac{x_{2}^{\prime} N_{x^{\prime}}-x_{2} N_{x}}{N_{i_{s}}} .
\end{gathered}
$$

Aus den beiden Gleichungen (I8) und (I9) lassen sich die gesuchten Grössen $Y_{S}$, der «echte» Ausnützungsfaktor, und die Menge $i_{s}$ unzersetzter Schwebestoffe des Rohwassers im Überschußschlamm mit Hilfe messbarer Grössen ermitteln. Für die beiden Unbekannten ergeben sich folgende Gleichungen:

und

$$
Y_{S}=\frac{x_{2}^{\prime}\left(N_{i_{S}}-N_{x^{\prime}}\right)}{\left(s_{R}-s\right)\left(N_{i_{s}}-N_{x^{\prime}}\right)+x_{2}^{\prime}\left(N_{x}-N_{x^{\prime}}\right)}
$$

$$
\begin{aligned}
i_{s}^{2}\left(N_{x}-N_{i_{s}}\right)+i_{s}\left[\left(s_{R}-s\right)\left(N_{i_{s}}-N_{x}\right)+x_{2}^{\prime}\left(N_{x}-N_{x}\right)\right] \\
-x_{2}^{\prime}\left(N_{x}-N_{x}\right)\left(s_{R}-s\right)=0 .
\end{aligned}
$$

$\left(s_{R}-s\right)$ ist die gesamte Konzentrationsabnahme des Abwassers, gemessen als org. C, bei der biologischen Reinigung und ist für einen schwebstofffreien Abfluss zu bestimmen (die Schwebestoffe des Abflusses sind ein Teil des Überschußschlamms $x_{2}^{\prime}$ ). Für den Stickstoffgehalt $N_{x}$ eines reinen Bakterienbelebtschlamms (ohne primäre Schwebestoffe) und denjenigen der langsam angreifbaren Schwebestoffe des Rohwassers $N_{i_{s}}$ muss man Schätzungen vornehmen. Wir haben für unsere Berechnungen folgende Werte angenommen:

$$
\begin{array}{ll}
N_{x}=0,07 & {[\mathrm{~g} \mathrm{~N} / \mathrm{g} \mathrm{TS}],} \\
N_{i_{s}}=0.02 & {[\mathrm{~g} \mathrm{~N} / \mathrm{g} \mathrm{TS}] .}
\end{array}
$$

Die Bestimmung von $Y_{S}$ und $i_{s}$ wurde für alle 27 Sommerversuche einzeln durchgeführt und ergibt folgendes Resultat: 
Grösse des "biologiscben» Ausnützungskoeffizienten $Y_{S}$ : Die Varianzanalyse der Werte zeigt, dass erwartungsgemäss weder die hydraulische Raumbelastung noch die Schlammkonzentration im Belüftungsbecken einen erkennbaren Einfluss auf die Ausnützung des biologisch angreifbaren Substrats für die Belebtschlammbildung ausüben. Hingegen ergibt sich ein deutlicher, wenn auch nur schwach gesicherter Abfall von $Y_{S}$ mit zunehmender O-Spannung im Belüftungsbecken. Diese letztere Beobachtung stimmt mit den Feststellungen für den scheinbaren Ausnützungskoeffizienten $Y_{S}^{\prime}$ (Tab. I2) überein. Die dort zusätzlich nachgewiesene Abhängigkeit des Ausnützungsgrades von der Schlammkonzentration ist offenbar auf den Einfluss der Schwebestoffe des Rohwassers zurückzuführen.

Tabelle 14

"Biologischer" Ausnützungskoeffizient $Y_{S}$ für den Schlammzuwachs im Belüftungsbecken a) Varianzanalyse

\begin{tabular}{lrrrrr}
\hline Variabilität & Fg & SQ & DQ & F & P \\
\hline$R_{h}$ & 2 & 54721 & 27360 & 0,71 & $<0,5>0,3$ \\
$x_{1}^{\prime}$ & 2 & 57724 & 28862 & 0,75 & $<0,5>0,3$ \\
O & 2 & 148726 & 74363 & 1,92 & $<0,3>0,1$ \\
Rest & 20 & 770314 & 38515 & & \\
Total & 26 & 1031485 & - & & \\
\hline
\end{tabular}

b) Mittelwerte von $Y_{S}$ ubber alle Raumbelastungen und Schlammkonzentrationen

\begin{tabular}{lll}
\hline $\mathrm{O}_{2}$ im Belüftungsbecken, $\mathrm{mg} / \mathrm{l}$ & $Y_{S}$ & Vertrauensgrenze für $\mathrm{P}=0,1$ \\
\hline 1 & 0,685 & \\
4 & 0,581 \\
7 & 0,504 & 0,122 \\
Gesamtmittel (27 Versuche) & 0,590 & 0,070 \\
\hline
\end{tabular}

Eine gegebene Menge biologisch eliminierten organischen Koblenstoffs ergibt also mit zunehmender $\mathrm{O}_{2}$-Spannung im Belüftungsbecken eine abnebmende Menge neu gebildeter Organismensubstanz. Bei $7 \mathrm{mg} O \mathrm{im}$ Belüftungsbecken wird ziemlich genau die Hälfte, bei maximal I mg $\mathrm{O} / 1$ dagegen $\mathrm{rd} .2 / 3$ der angreifbaren organischen $\mathrm{C}$-Verbindungen (als org. C gemessen), die eliminiert werden, in neuen Belebtschlamm übergeführt. Eine physiologische Erklärung dieser Beobachtung ist nicht ohne weiteres möglich, denn im Wert $Y_{S}$ ist neben dem Organismenzuwachs auch der Organismenverlust durch Autolyse oder andere Faktoren (Predatoren) enthalten. Da mit ziemlicher Sicherheit 
die Sauerstoff konzentration im Bereich von I-7 mg O/l (auch unter Berücksichtigung kleinerer O-Spannungen im Innern von Flocken) für die Substratausnützung keine Rolle spielt, und auch das Ausmass der Autolyse kaum vom Sauerstoffgehalt des Milieus abhängt, ist die Rolle der Predatoren für den Zellverlust zu prüfen. Schon VIEHL [I 5 ] hatte beobachtet, dass in seinen Versuchsanlagen bei Sauerstoffkonzentrationen um I mg/l eine signifikant geringere Zahl von Ziliaten im Belebtschlamm vorhanden war als bei $5-6 \mathrm{mg} / \mathrm{l}$. Wir haben beim vorliegenden Faktorenversuch eine analoge Fesistellung gemacht, indem besonders bei den Experimenten mit $7 \mathrm{mg} \mathrm{O} / 1$ im Beliuftungsbecken, die Ziliatenpopulation ganz erheblich dichter war als bei $4 \mathrm{mg} \mathrm{O} / \mathrm{l}$ und besonders bei $\mathrm{I} \mathrm{mg} \mathrm{O} / \mathrm{l}$ (Ziliaten nur vereinzelt vorkommend). Da diese Einzeller einerseits beachtliche Konsumenten von Bakterien sind und anderseits bei ihrer Autolyse praktisch keine festen Rückstände verbleiben, wäre eine Wechselwirkung zwischen Feststoff bildung und Sauerstoffgehalt gemäss Tab. I $4 \mathrm{~b}$ (wenn auch indirekt) gegeben. Wir werden auf diesen Zusammenhang in einer späteren Arbeit bei der Auswertung der biozönologischen Verhältnisse in den Belebtschlämmen der Versuche nochmals zurückkommen.

Primäre Schpwebestoffe im Überschußscblamm $i_{s}$ : Da die absolute Menge der vom Rohwasser in den Überschußschlamm übergehenden Schwebestoffe natürlich stark vom Feststoffgehalt des Rohwassers abhängt, drückt man die Werte von $i_{s}$ besser in \% des Schwebestoffgehaltes des Rohwassers als in $\mathrm{g} / \mathrm{m}^{3}$ aus. Die 27 Sommerversuche zeigen dann gemäss Varianzanalyse in Tab. I5a:

Der Prozentanteil von $i_{s}$ im Überscbußschlamm ist pon der bydraulischen Raumbelastung und der O-Spannung im Belïftungshecken unabbängig, nimmt jedoch mit wacbsender Scblammkonzentration stark ab.

Tabelle 15

Prozent primäre Schwebestoffe $i_{s}$ im Überschußschlamm

a) Varianzanalyse

\begin{tabular}{lrrrrl}
\hline Variabilität & Fg & SQ & DQ & \multicolumn{1}{l}{ F } & P \\
\hline$R_{h}$ & 2 & 91,89 & 45,94 & \multicolumn{1}{l}{0,27} & $\geqslant 0,3$ \\
$x_{1}$ & 2 & 5458,94 & 2729,47 & 15,99 & $<0,001$ \\
O & 2 & 703,34 & 351,67 & 2,06 & $<0,3>0,1$ \\
Rest *) & 20 & 3419,58 & 170,97 & & \\
Insgesamt & 26 & 9673,75 & - & & \\
\multicolumn{7}{l}{ *) Wechselwirkungen nicht signifikant } \\
\hline
\end{tabular}


Tabelle 15 (Fortsetzung)

b) Mittelwerte des prozentualen Anteils der Schwebestoffe im Anlagenzufluss, die bei den Raumbelastungen 9-58 $\mathrm{m}^{3} / \mathrm{m}^{3} \cdot$ Tag (je 3 Experimente pro Mittelwert) im Úber-
schußschlamm erscheinen

\begin{tabular}{|c|c|c|c|c|}
\hline $\begin{array}{l}O \\
x_{1}^{\prime}\end{array}$ & 1 & 4 & 7 & $\begin{array}{l}\text { Mittelwert über alle O-Konzentrationen } \\
\text { (je } 9 \text { Experimente) }\end{array}$ \\
\hline $\begin{array}{r}600 \\
3300 \\
6000\end{array}$ & $\begin{array}{l}54,2 \\
25,3 \\
16,6\end{array}$ & $\begin{array}{r}34,5 \\
22,6 \\
9,8\end{array}$ & $\begin{array}{r}44,8 \\
9,5 \\
6,6\end{array}$ & $\begin{array}{r}44,5 \\
19,1 \\
6,6\end{array}$ \\
\hline
\end{tabular}

Vertrauensgrenzen für $P=0,1$ bei Mittelwerten aus je 3 Experimenten $= \pm 14,14$, a us je $9 \mathrm{Ex}$ perimenten $= \pm 8,18$, aus je 27 Experimenten $= \pm 4,70$

Der Einfluss der Schlammkonzentration auf den Abbau der primären Schwebestoffe ist offensichtlich sehr gross, insbesondere bei hohen Sauerstoffspannungen. Wenn man den Begriff des Schlammalters $x_{1}^{\prime} / R_{h} x_{2}^{\prime}$ beizieht, kann man auch sagen, dass mit zunehmendem Scblammalter die primären Schpobestoffe im Belebtschlamm immer mebr verscbwinden und ebenfalls in den Stoffmechsel des Belebtschlamms einbezogen merden.

Die Berechnungen für $Y_{S}$ und $i_{s}$ haben wir nur für die Sommerversuche vorgefuihrt. Grundsätzlich zeigt sich bei niedrigeren Temperaturen nichts Neues, obschon die absoluten Werte für $Y_{S}$ und $i_{s}$ sich gegenüber den vorstehenden Angaben etwas verschieben.

\section{Diskussion der Ergebnisse}

\subsection{Reinigungseffekt und Schlammbelastung}

Mit Gleichung (12a) wurde gezeigt, dass der Reinigungseffekt $\eta$ angenähert umgekehrt proportional zur Schlammbelastung ist, wenn man eine nicht zu grosse Variabilität des Quotienten $A / Y_{S}$ voraussetzt. Wir haben in Abb. 3 die beobachteten Reinigungseffekte in Funktion der Schlammbelastung aufgetragen (die Beobachtungen der Versuche mit verschiedenen Sauerstoffspannungen, aber gleicher Raumbelastung und Schlammkonzentration wurden zu einem einzigen Wert zusammengefasst). Die Korrelation ergibt stetige Kurven, deren Lage für die beiden Temperaturblöcke erwartungsgemäss etwas verschieden ist. Der allgemeine Verlauf der Kurven stimmt mit der algebraischen Formulierung von (12a) überein. Die einzelnen untersuchten hydraulischen Raumbelastungen fallen unabhängig von ihrer Grösse erwartungsgemäss auf dieselbe Kurve, da ja bei gegebener Abwasser- und Schlammkonzentration die Raumbelastung und Schlammbelastung miteinander verbunden sind. 


\section{$\eta \%$}

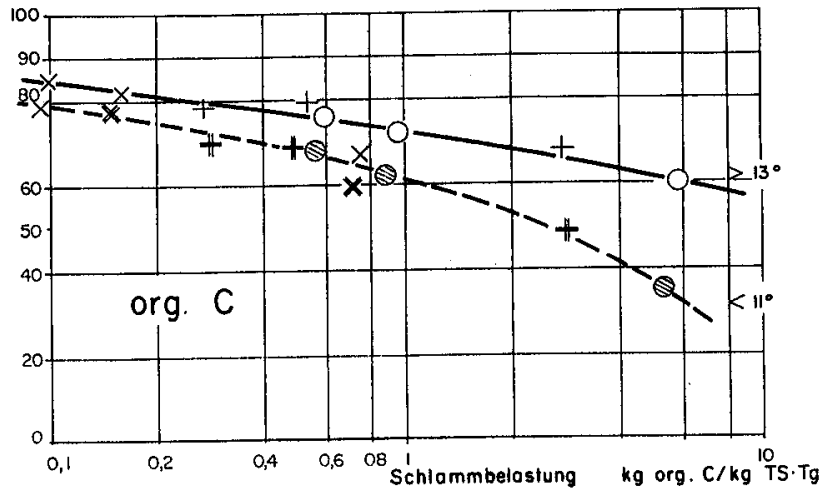

\section{$\eta \%$}

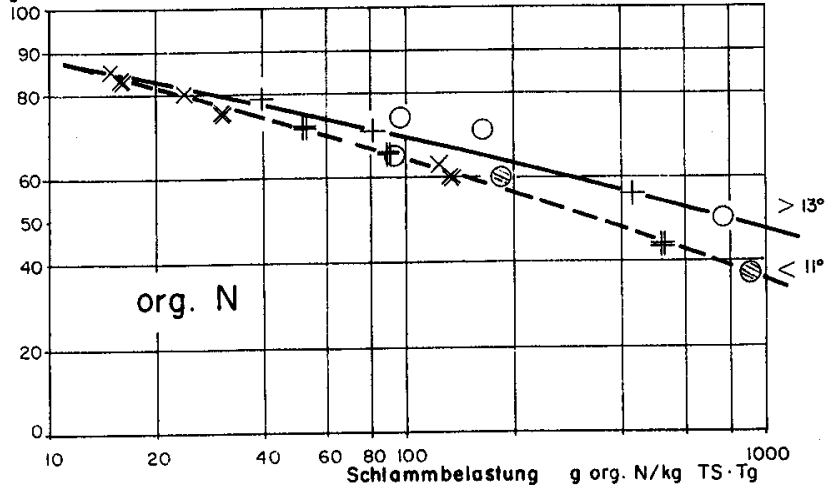

\section{$\eta \%$}

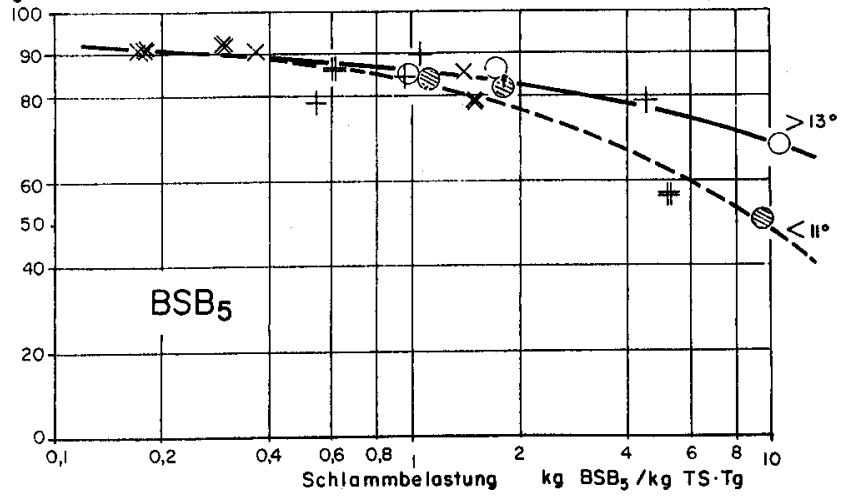

Abbildung 3

Reinigungseffekte bezüglich org, $\mathrm{C}$, org. $\mathrm{N}$ und $\mathrm{BSB}_{5}$ in Funktion der Schlammbelastung (berechnet mit dement$>13^{\circ} \mathrm{C}<11^{\circ} \mathrm{C}$

$$
\begin{array}{rll}
R_{h} \approx 58 \mathrm{~m}^{3} / \mathrm{m}^{3} \cdot \mathrm{Tg} & \bigcirc & \\
29 \mathrm{~m}^{3} / \mathrm{m}^{3} \cdot \mathrm{Tg} & + & \# \\
9 \mathrm{~m}^{3} / \mathrm{m}^{3} \cdot \mathrm{Tg} & \times & \#
\end{array}
$$
sprechenden Analysenparameterund derTemperatur). Jeder Punkt ist der Mittelwert aus drei Experimenten. 
Angaben von Reinigungseffekten in Funktion der Schlammbelastung, bezogen auf org. $\mathrm{C}$ und org. $\mathrm{N}$, sind bisher nicht veröffentlicht worden, hingegen liegt eine Reihe von Arbeiten vor, in denen der Reinigungseffekt in Abhängigkeit der "Schlammbelastung mit $\mathrm{BSB}_{5}$ " dargestellt wurde (vgl. Zusammenstellung aus früheren Arbeiten bei v.D.EMDE [3], und eine Kompilation neuerer Werte bei KL. IMHOFF [8]). Wenn auch in der Grössenordnung etwa übereinstimmend, weichen doch die bisher veröffentlichten $\mathrm{BSB}_{5}$-Kurven nicht unerheblich von unseren Beobachtungen ab. Ein wesentlicher Grund für die Unterschiede liegt darin, dass in den bisherigen Arbeiten die Temperatur nicht berücksichtigt ist. Sowohl die von v.D.EMDE als auch die von IMHOFF gegebenen Darstellungen stimmen im übrigen nicht mit dem mathematisch geforderten allgemeinen Verlauf der Kurven überein. Da es sich in beiden Fällen um Kompilationen von Beobachtungen an Anlagen ganz unterschiedlicher Betriebsbedingungen handelt, ist diese Abweichung erklärlich.

Die Beziehungen in Abb. 3 sind nicht von der Abwasserkonzentration abhängig, soweit gemäss Gl. (I2a) der Ausnützungskoeffizient $Y_{s}^{\prime}$ unberührt bleibt. Dies dürfte im allgemeinen für Abwasser städtischen Charakters zutreffen, so dass die vorliegenden Kurven eine brauchbare Dimensionierungsgrundlage darstellen.

\subsection{Die sogenannte "Restperschmutzung" in den Nacbklärbecken- abflüssen}

Die Kurven von Abb. 3, die sich auf die effektiv gemessenen Konzentrationen in den unfiltrierten Nachklärbeckenabflüssen beziehen, zeigen, dass sowohl beim org. $\mathrm{C}$ als auch beim org. $\mathrm{N}$ die Elimination nicht höher als rd. $85 \%$ (auch bei den kleinsten geprüften Schlammbelastungen) anstieg. Die verbleibenden rd. $15 \%$, die als Restverunreinigung in die Vorfluter gelangen, beanspruchen eine gewisse Aufmerksamkeit, da diese Restverunreinigung in absoluten Quantitäten proportional mit den an eine Kläranlage angeschlossenen Einwohnern zunimmt und eventuell in der Zukunft erneut zu untragbaren Vorflutbelastungen führen kann. Wir haben deshalb zu prüfen, wie sie zustande kommt.

Aus analytischen Gründen haben wir die Nachklärbeckenabflüsse nicht nach Filtration untersucht, sondern den indirekten Weg beschritten, aus den abfliessenden Mengen gesamten org. $C$ und dem org. $C$ der suspendierten Stoffe die verbleibende Menge gelöster org. C-Verbindungen zu errechnen. 


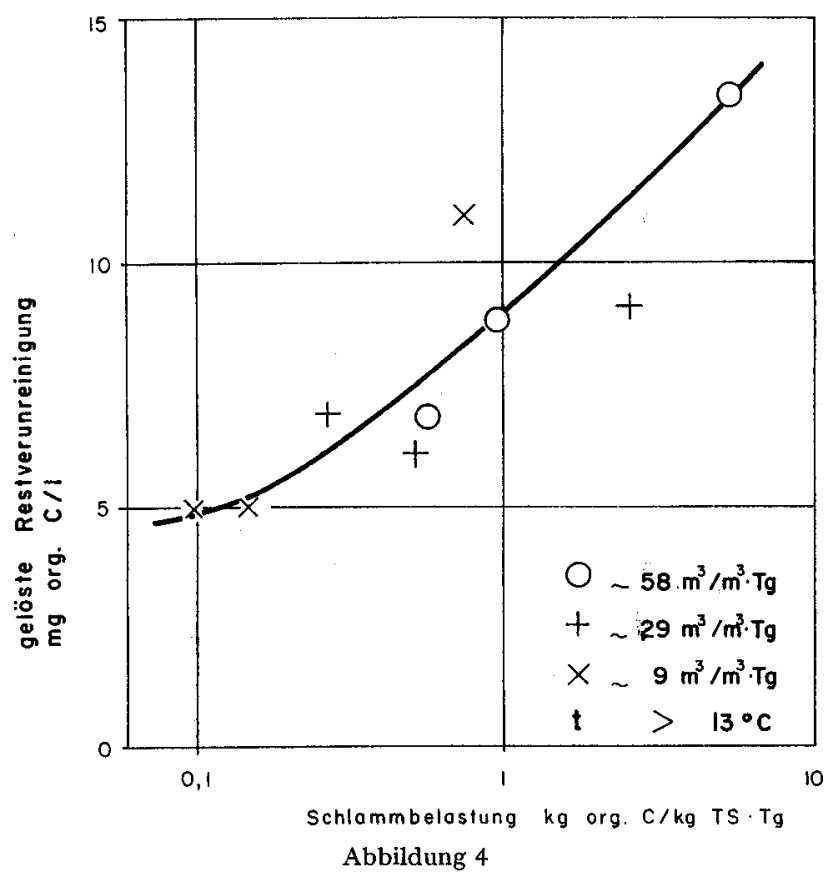

Gehalt an gelösten org. C-Verbindungen in den Abflüssen der Nachklärbecken (Sommerversuche) in Funktion der Schlammbelastung mit org. C. Mittelwerte von je 3 Experimenten (Sauerstoffstufen).

Da es bei der Restverunreinigung nur auf die absoluten Werte ankommt, geben wir in Abb. 4 die beobachteten Konzentrationen in $\mathrm{mg} / \mathrm{l}$ bei den Sommerversuchen in Funktion der Schlammbelastungen wieder. Eine Abhängigkeit der Werte vom Sauerstoffgehalt im Belüftungsbecken war wiederum nicht signifikant, so dass wir Mittelwerte aus je 3 Versuchen (analog wie in Abb. 3) bilden konnten. Die Darstellung zeigt sofort, dass die sog. Restverunreinigung, die in gelöster Form vorliegt und damit nicht vom zufälligen Schwebestoffgehalt des Nachklärbeckenabflusses abhängig ist, im ganzen untersuchten Bereich mit steigender Raumbelastung stetig zunimmt, $\mathrm{d}$.h. also aller Wahrscheinlichkeit nach aus Verbindungen besteht, die unter den gewählten Betriebsbedingungen noch "abbaubar" wären. Eine Abflachung der Kurve ist zwar bei Schlammbelastungen unter etwa $0,2 \mathrm{~kg}$ org. $\mathrm{C} / \mathrm{kg}$ TS - Tag angedeutet, doch fehlen Beobachtungen bei noch viel kleineren Belastungen, um ein Urteil über einen eventuellen "konstanten» Endwert an Restkohlenstoff fällen zu können. Aus Abb. 4 ist immerhin ersichtlich, dass auch bei Erzielung eines "vollständig schwebestofffreien Abflusses" immer noch organische $\mathrm{C}$-Verbindungen im gereinigten Abwasser verblei- 

ben, deren Menge in der Grössenordnung von 10\% des C-Gehaltes vorge-
klärten Abwassers liegt.

HUNKEN [7] (vgl. dort auch ältere Literatur) hat einen «Endwert» der Reinigung häuslichen Abwassers in statischen Versuchen bestimmt, der in der Grösse von 35\% der Oxydierbarkeit vorgeklärten Abwassers liegt. Eine "Umrechnung" des $\mathrm{KMnO}_{4}$-Verbrauches von Abwasser in organischen Kohlenstoff ist nicht möglich, so dass ein Vergleich der Beobachtungen von HUNKEN mit unseren Werten schwierig ist. Immerhin scheint uns, dass die Untersuchung von HUNKEN doch ein zu pessimistisches Bild von der Höhe der Restverunreinigung im Abfluss einer biologischen Anlage für häusliches Abwasser vortäuscht. Wir sind trotzdem der Ansicht, dass im Hinblick auf die zukünftige Entwicklung der Bevölkerungszahlen und der Industrialisierung dem Problem der Restverunreinigung die grösste Aufmerksamkeit zu schenken ist. Seine Lösung ist aber auf Grund der vorstehenden Versuchsergebnisse mit biologischen Reinigungsstufen allein nicht zu erreichen, sondern erfordert zusätzliche Massnahmen (chemisch-physikalische Nachreinigungsverfahren).

\subsection{Zusammenfassende Beurteilung des Temperatureinflusses}

Die Temperaturmittelwerte (Belüftungsbecken) für die beiden Versuchsblöcke $\left\langle\right.$ II ${ }^{\circ} \mathrm{C}$ und $>\mathrm{I}_{3}{ }^{\circ} \mathrm{C}$ betragen $10^{\circ} \mathrm{C}$, bzw. $16,3^{\circ} \mathrm{C}$. Es kam also durchschnittlich eine Temperaturdifferenz von $6,3^{\circ} \mathrm{C}$ auf alle temperaturabhängigen Reaktionen in den Belüftungsbecken zur Wirkung.

Aus den Angaben in Abschnitt 4 über die Reinigungseffekte in den Winter- und Sommerversuchen lässt sich für vergleichbare Betriebsbedingungen die Proportion $\eta_{10^{\circ}} / \eta_{16,3^{\circ}}$ ableiten. In Tab. I6 sind für die drei untersuchten Komponenten die entsprechenden Quotienten zusammengestellt.

Eine Wirkung der Temperatur auf den Reinigungseffekt irgendeiner Komponente des Abwassers ist ganz allgemein nur dann zu erwarten, wenn diese Komponente in geschwindigkeitsbegrenzender Menge für den Stoffwechsel der Organismen im untersuchten Temperaturbereich vorhanden

Tabelle 16

Temperatureinfluss auf den Reinigungseffekt als Quotient $\eta_{10^{\circ}} / \eta_{16,3^{\circ}}$

\begin{tabular}{|c|c|c|c|c|c|c|c|c|c|}
\hline \multirow[b]{2}{*}{$\begin{array}{l}R_{h} \\
x_{1}^{\prime \prime}\end{array}$} & \multicolumn{3}{|c|}{$\eta_{\mathrm{C10}} / \eta_{\mathrm{C}_{16,3}}$} & \multicolumn{3}{|c|}{$\eta_{\mathrm{N} 10} / \eta_{\mathrm{N}_{16,3}}$} & \multicolumn{3}{|c|}{$\eta_{\mathrm{B} 1 \mathrm{O}} / \eta_{\mathrm{B} 16,3}$} \\
\hline & 58 & 29 & 10 & 58 & 29 & 10 & 58 & 29 & 10 \\
\hline $\begin{array}{r}600 \\
3300 \\
6000\end{array}$ & $\begin{array}{l}0,58 \\
0,85 \\
0,91\end{array}$ & $\begin{array}{l}0,72 \\
0,86 \\
0,90\end{array}$ & $\begin{array}{l}0,90 \\
0,95 \\
0,93\end{array}$ & $\begin{array}{l}0,73 \\
0,84 \\
0,87\end{array}$ & $\begin{array}{l}0,79 \\
0,93 \\
0,91\end{array}$ & $\begin{array}{l}0,95 \\
0,93 \\
0,97\end{array}$ & $\begin{array}{l}0,73 \\
0,95 \\
0,99\end{array}$ & $\begin{array}{l}0,72 \\
0,94 \\
0,99\end{array}$ & $\begin{array}{l}0,92 \\
1 \\
1\end{array}$ \\
\hline
\end{tabular}


ist. Beispielsweise lässt sich bei hoher Temperatur die beschleunigte Elimination eines Substrats nicht beobachten, wenn es unter den gegebenen Betriebsbedingungen hinsichtlich Schlammkonzentration und Belüftungszeit bereits bei niedriger Temperatur vollständig eliminiert wird. Eine Anlage muss deshalb grundsätzlich je nach Belastungszustand ungleich stark auf Temperatureinflüsse reagieren, und im speziellen kann die Temperatur bei der Reinigung ein und desselben Abwassers bei gegebener Belüftungszeit und Schlammkonzentration auf den Reinigungseffekt der einzelnen Komponenten eine unterschiedliche Wirkung ausüben. Die Ziffern in Tab. I6 beweisen diesen Zusammenhang, der auch aus älteren Untersuchungen (vgl. z.B. die Versuchsreihe von VIEHL und MeIssNer [I6], Messung des org. N; oder das Experiment von SAWYER et al. [II], Messung des $\mathrm{BSB}_{5}$ ) deutlich hervorgeht. Anderseits ist es auch leicht einzusehen, dass im allgemeinen bei Anlagen der Praxis mit ihren geringen Belastungen die beobachteten Temperaturwirkungen nur klein sind. Tab. I6 zeigt aber doch, dass bei "hochbelasteten" Belebtschlammanlagen, bzw. zu jenen Tageszeiten, wo eine Anlage relativ hoch belastet ist, niedere Temperaturen sich sofort in einer nicht zu vernachlässigenden Verminderung des Reinigungseffektes am org. $\mathrm{C}$, org. $\mathrm{N}$ und $\mathrm{BSB}_{5}$ ausdrücken. Die beobachteten Unterschiede im Reinigungseffekt beim org. $\mathrm{C}$ und org. $\mathrm{N}$ zwischen $\mathrm{rd}$. 16, bzw. $10^{\circ} \mathrm{C}$ erscheinen uns so gross, dass sie für Dimensionierungsberechnungen nicht unberücksichtigt bleiben dürfen. Dieser Gesichtspunkt sollte besonders bei Anlagen in Regionen mit sehr niedrigen Wintertemperaturen des Abwassers nicht übersehen werden.

\subsection{Korrelation pon Überscbußscblammproduktion und Abbauleistung des Belebtscblamms}

Gemäss der Theorie ist die Überschußschlammproduktion nicht von der Schlammkonzentration $x_{1}^{\prime}$ im Belüftungsbecken abhängig. Hingegen tritt $x_{1}^{\prime}$ in die US-Beziehung ein, sobald man die Autolyse und die Predatorwirkungen berücksichtigt (Gl. I6). Man kann deshalb mit einer gewissen Berechtigung, wenn auch nur auf rein empirischer Basis, die USS-Produktion mit der spezifischen Abbauleistung des Schlamms in den einzelnen Versuchen korrelieren und erhält dann Abb. 5. Die Linearität der Abhängigkeit der Schlammproduktion von der Abbauleistung ergibt folgende Formulierung (alle Grössen mit dem Mass org. C):

$$
\begin{gathered}
\frac{\sigma\left(s_{R}-s\right)}{x_{1}^{\prime}}=x_{2}^{\prime} \quad\left[\mathrm{g} \text { org. } \mathrm{C} / \mathrm{m}^{3} R W\right], \\
\sigma=f\left(x_{1}^{\prime}, t^{\circ}\right) \quad\left[\mathrm{g} \text { org. } \mathrm{C} / \mathrm{m}^{3}{ }_{B B}\right] .
\end{gathered}
$$




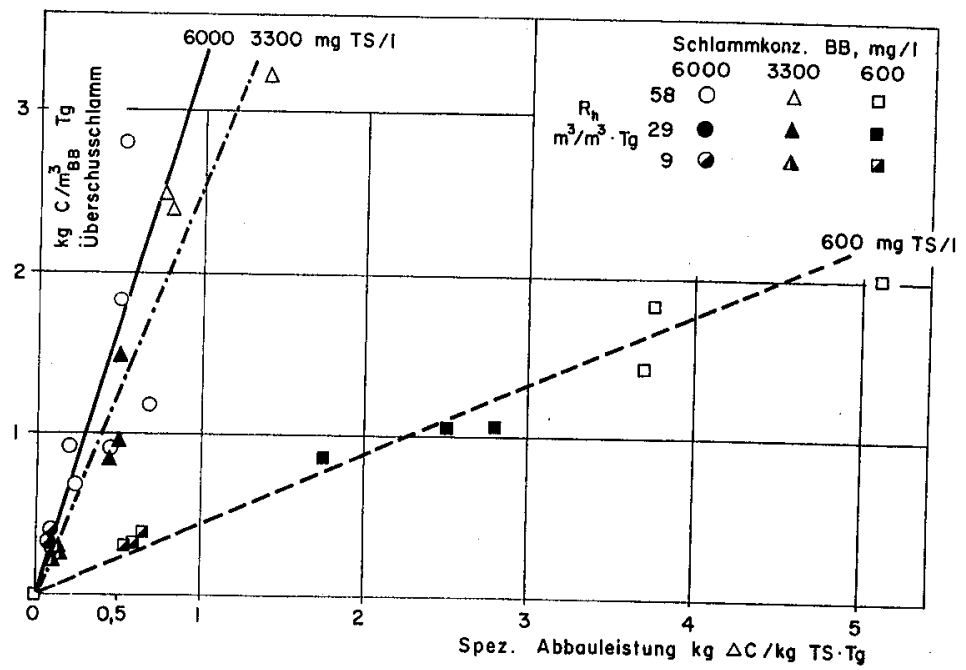

Abbildung 5

Überschußschlammenge (inklusive Schwebestoffe im Nachklärbeckenabfluss) in Funktion der spez. Abbauleistung des Schlamms bei drei Belebtschlammkonzentrationen. Sommerversuche, $t>13^{\circ} \mathrm{C}$.

Da $x_{2}^{\prime} /\left(s_{R}-s\right)$ unserem früher verwendeten "Brutto»ausnützungskoeffizienten $Y_{S}^{\prime}$ entspricht, sagt die Beziehung (22) nur aus, dass $Y_{S}^{\prime}$ für ein und dieselbe Schlammkonzentration unveränderlich ist, aber mit der Schlammkonzentration (und auch mit der Temperatur) wechseln kann. Dies wurde schon aus den Ziffern in Tab. 12 festgestellt.

Für die Konstante $\sigma$ ergeben sich aus unseren Beobachtungen folgende Werte:

Tabelle 17

Produktionskonstante $\sigma$ für den Überschußschlamm; Mittelwerte über die Raumbelastungen 9-58 $\mathrm{m}^{3} / \mathrm{m}^{3} \cdot$ Tag und die Sauerstoffkonzentration 1-7 mg/l im Belïftungsbecken

\begin{tabular}{ccc}
\hline $\operatorname{g~TS}_{1}^{\prime} / \mathrm{m}^{3}{ }_{B B}$ & $<11^{\circ} \mathrm{C}$ & $>13^{\circ} \mathrm{C}$ \\
\hline 600 & 183 & 164 \\
3300 & 924 & 793 \\
6000 & 1219 & 1161 \\
\hline
\end{tabular}

Mit dieser Beziehung lässt sich die ÜS-Produktion abschätzen (nach Abzug der Schwebestoffe im Nachklärbeckenabfluss!), wenn man die Betriebsbedingungen einer Anlage auf Grund des geforderten Reinigungsgrades festgelegt hat. Sie trägt als rein empirische Formel allen nicht einzeln messbaren Einflussgrössen auf die ƯS-Bildung Rechnung, gilt aber 
selbstverständlich auch nur für den von uns untersuchten Bereich von Betriebsbedingungen.

Die beschriebenen Untersuchungen haben die biologische, chemische und technische Abteilung der EAWAG während längerer Zeit intensiv beschäftigt, und es ist nur der gleichbleibenden sorgfältigen Hilfe aller beteiligten Mitarbeiter zu danken, wenn das Beobachtungsmaterial über diesen mehrjährigen Zeitraum mit vergleichbarer Qualität gesammelt werden konnte. Ich möchte speziell meinen beiden Kollegen, Herrn Dr. E. MÄrKI, Chef der chemischen Abteilung, und Herrn Prof. A.HörLER, Chef der technischen Abteilung, für ihre Mithilfe danken. Speziell zu danken ist sodann Herrn FRIEDRICH, welcher als Betriebsleiter der Versuchsanlage die Durchführung der Experimente überwachte und die gesamte Erhebung der Betriebsdaten zu besorgen hatte.

\section{Summary}

Results of pilot plant experiments on a technical scale with the activated sludge process are presented. The investigation was conducted on the basis of a complete block factorial design where hydraulic load, mixed liquor solids concentration and $\mathrm{O}_{2}$-tension in the mixed liquor were independant variables, each of which was tested at three levels. In addition, the entire set of $3^{3}$ experiments was repeated within two temperature regions $\left(>\mathrm{I}_{3}{ }^{\circ} \mathrm{C}\right.$ and $\left\langle\mathrm{II}{ }^{\circ} \mathrm{C}\right)$ of the mixed liquor. Thus, 54 experiments are available for evaluation (experimental details see Chapter 3 ). The present article discusses the main actions and interactions of the independant variables on removal of organic carbon, organic nitrogen and BOD, as well as on the production of excess sludge. The evaluation is based on the theory of continuous fermentation in a completely mixed reactor (Chapter 2).

The actions of the four independant variables on purification effects are shown by the analysis of variance of the experimental observations (Chapter 4). Elimination of pollution was significantly higher in the temperature region of $>\mathbf{I} 3^{\circ} \mathrm{C}$ than below ${ }{ }^{\circ}{ }^{\circ} \mathrm{C}$ at high plant loads. However, the effect of temperature diminished markedly with decreasing loads (other conditions being equal). As expected, the mixed liquor concentration and the detention time exerted a pronounced effect on the degree of purification (Fig. I). The relationship is best expressed by the (theoretically justified) combined parameter of sludge load (Fig. 3).

Oxygen tension in the mixed liquor was of no significance within the limits investigated $(\mathrm{I}-7 \mathrm{ppm})$ for the efficiency of pollution removal.

The "threshold" pollution of the purified effluents (in terms of dissolved organic carbon compounds) has been found decreasing with decreasing sludge load (Fig. 4). At less than $0,1 \mathrm{~kg}$ org. $\mathrm{C} / \mathrm{kg}$ solids per day it was still around $10 \%$ of the value in sedimented raw sewage. This demonstrates clearly that the remaining pollution consists (at least partially) of slowly decomposable compounds which may not even be removed at extremely low plant loads. 
The solids production (excess sludge plus solids in the effluent of the final clarifier) was primarily expressed as an overall "yield factor", i.e. in terms of organic carbon removed from the sewage. The magnitude of this factor is a complicated function of many influences such as: solids in the incoming sewage, amount of decomposition of these solids, autolysis of organisms, predator effects by protozoans, temperature, etc. Average values of 0.73 and $0.66 \mathrm{~g}$ org. $\mathrm{C} / \mathrm{g}$ org. Celiminated were found at $\angle I I{ }^{\circ} \mathrm{C}$ and $>13^{\circ} \mathrm{C}$ respectively. A statistically significant decrease of the yield factor with increasing mixed liquor solids and $\mathrm{O}_{2}$-concentration was observed. The proportion of primary suspended solids in the excess sludge at different operation conditions could be calculated and a "true" biological yield factor was estimated. The form for a crude estimation of excess sludge production in terms of BOD removed reads $x_{2}^{\prime}=$ $0.85 s_{R}-0.45 s-20$ [g excess sludge solids per $\mathrm{m}^{3}$ of sewage treated], where $s_{R}$ and $s$ are BOD values of the incoming and treated sewage and the last term is an average value of suspended solids in the final effluent.

\section{Tabellenanhang}

Die nachfolgenden Tab. I 8-34 enthalten die Mittelwerte der Beobachtungen innerhalb der Analysenperioden jedes einzelnen Experimentes samt den Standardabweichungen dieser Mittelwerte, soweit sie von Interesse sind. Die Anzahl der in jedem Mittelwert enthaltenen Einzelbeobachtungen ist aus Raumgründen nicht angegeben. Sie beträgt für alle Analysendaten (Tab. 30-34) $N=9$ (vgl. auch S. 229). Die Mittelwerte der Betriebsdaten (Tab. I 8-2I) sind - je nach Länge einer Versuchsperiode aus 20 bis 30 Einzelbeobachtungen gebildet. Die unabhängigen Variablen Raumbelastung, Schlammkonzentration und Sauerstoffkonzentration sind mit gerundeten Werten als Tabelleneingänge verwendet. Die in den einzelnen Experimenten effektiv eingehaltenen Werte dieser Betriebsgrössen sind aus den Tab. I 8, I9 und 20 ersichtlich. Es sind nachstehend nur jene Beobachtungen tabelliert, die im Zusammenhang mit den Ausführungen in diesem Aufsatz wesentlich sind. Weitere Angaben aus diesem Faktorenversuch werden wir bei der Diskussion anderer Fragestellungen bringen.

Tabelle 18

Hydraulische Raumbelastung $\quad\left[\mathrm{m}^{3} / \mathrm{m}^{3} B B \cdot \mathrm{Tag}\right]$

\begin{tabular}{|c|c|c|c|c|c|c|c|}
\hline \multirow{3}{*}{$\begin{array}{l}\mathrm{O}_{2}-\mathrm{Konz} \\
\mathrm{BB} \\
\mathrm{mg} / \mathrm{l}\end{array}$} & \multirow{3}{*}{$\begin{array}{l}\text { Schlamm- } \\
\text { konz. BB } \\
\mathrm{mg} / 1\end{array}$} & \multirow{2}{*}{\multicolumn{3}{|c|}{$\begin{array}{l}\text { Mittlere Temperatur } \mathrm{BB}= \\
>13^{\circ} \mathrm{C} \\
\text { Hydraulische Raumbelastung } \\
\mathrm{m}^{3} / \mathrm{m}^{3} \cdot \text { Tag }\end{array}$}} & \multirow{2}{*}{\multicolumn{3}{|c|}{$\begin{array}{l}\text { Mittlere Temperatur } \mathrm{BB}= \\
<11^{\circ} \mathrm{C} \\
\text { Hydraulische Raumbelastung } \\
\mathrm{m}^{3} / \mathrm{m}^{3} \cdot \text { Tag }\end{array}$}} \\
\hline & & & & & & & \\
\hline & & 58 & 29 & 9 & 58 & 29 & 9 \\
\hline 1 & $\begin{array}{r}600 \\
3300 \\
6000\end{array}$ & $\begin{array}{l}58,2 \\
57,5 \\
57,0\end{array}$ & $\begin{array}{l}28,0 \\
28,8 \\
29,0\end{array}$ & $\begin{array}{l}8,7 \\
9,1 \\
9,1\end{array}$ & $\begin{array}{l}58,1 \\
57,7 \\
57,7\end{array}$ & $\begin{array}{l}29,1 \\
29,2 \\
29,4\end{array}$ & $\begin{array}{l}9,1 \\
9,1 \\
9,2\end{array}$ \\
\hline 4 & $\begin{array}{r}600 \\
3300 \\
6000\end{array}$ & $\begin{array}{l}57,4 \\
57,5 \\
56,9\end{array}$ & $\begin{array}{l}28,9 \\
28,9 \\
28,7\end{array}$ & $\begin{array}{l}9,1 \\
9,1 \\
9,2\end{array}$ & $\begin{array}{l}57,9 \\
57,8 \\
58,1\end{array}$ & 29,2 & $\begin{array}{l}9,1 \\
9,3 \\
9,3\end{array}$ \\
\hline 7 & $\begin{array}{r}600 \\
3300 \\
6000\end{array}$ & $\begin{array}{l}57,0 \\
57,1 \\
57,0\end{array}$ & $\begin{array}{l}28,7 \\
28,5 \\
28,5\end{array}$ & $\begin{array}{l}9,2 \\
9,1 \\
9,0\end{array}$ & $\begin{array}{l}58,3 \\
55,7 \\
57,0\end{array}$ & $\begin{array}{l}29,2 \\
28,9 \\
28,1\end{array}$ & $\begin{array}{l}9,3 \\
9,3 \\
9,1\end{array}$ \\
\hline
\end{tabular}


Tabelle 19

Schlammkonzentration im Belüftungsbecken [mg Trockensubstanz/1]

\begin{tabular}{|c|c|c|c|c|c|c|c|}
\hline \multirow{3}{*}{$\begin{array}{l}\mathrm{O}_{2}-\mathrm{Konz} . \\
\mathrm{BB} \\
\mathrm{mg} / 1\end{array}$} & \multirow{3}{*}{$\begin{array}{l}\text { Schlamm- } \\
\text { konz. BB } \\
\mathrm{mg} / 1\end{array}$} & \multirow{2}{*}{\multicolumn{3}{|c|}{$\begin{array}{l}\text { Mittlere Temperatur } \mathrm{BB}= \\
>13^{\circ} \mathrm{C} \\
\text { Hydraulische Raumbelastung } \\
\mathrm{m}^{3} / \mathrm{m}^{3} \cdot \text { Tag }\end{array}$}} & \multirow{2}{*}{\multicolumn{3}{|c|}{$\begin{array}{l}\text { Mittlere Temperatur } \mathrm{BB}= \\
<11^{\circ} \mathrm{C} \\
\text { Hydraulische Raumbelastung } \\
\mathrm{m}^{3} / \mathrm{m}^{3} . \text { Tag }\end{array}$}} \\
\hline & & & & & & & \\
\hline & & $\mathbf{5 8}$ & 29 & 9 & 58 & 29 & 9 \\
\hline 1. & 6000 & $\begin{array}{c}644 \\
\pm 56 \\
3256 \\
\pm 455 \\
5976 \\
\pm 260\end{array}$ & $\begin{array}{c}680 \\
\pm 180 \\
3390 \\
\pm 238 \\
6050 \\
\pm 260\end{array}$ & $\begin{array}{c}645 \\
\pm 88 \\
3405 \\
\pm 113 \\
6020 \\
\pm 114\end{array}$ & $\begin{array}{c}661 \\
\pm 98 \\
3392 \\
\pm 157 \\
5743 \\
\pm 160\end{array}$ & $\begin{array}{c}689 \\
\pm 104 \\
3332 \\
\pm 214 \\
6104 \\
\pm 205\end{array}$ & $\begin{array}{c}656 \\
\pm 95 \\
3342 \\
\pm 218 \\
5989 \\
\pm 134\end{array}$ \\
\hline 4 & 3300 & $\begin{array}{c}702 \\
\pm 52 \\
3310 \\
\pm 250 \\
5995 \\
\pm 315\end{array}$ & $\begin{array}{c}670 \\
\pm 76 \\
3366 \\
\pm 120 \\
5896 \\
\pm 82\end{array}$ & $\begin{array}{c}643 \\
\pm 71 \\
3278 \\
\pm 73 \\
6083 \\
\pm 330\end{array}$ & $\begin{array}{c}704 \\
\pm 33 \\
3421 \\
\pm 264 \\
6185 \\
\pm 333\end{array}$ & $\begin{array}{l}6158 \\
\pm 215\end{array}$ & $\begin{array}{c}678 \\
\pm 90 \\
3398 \\
\pm 59 \\
6000 \\
\pm 264\end{array}$ \\
\hline & 3300 & $\begin{array}{c}674 \\
\pm 68 \\
3416 \\
\pm 118 \\
6000 \\
\pm 272\end{array}$ & $\begin{array}{c}710 \\
\pm 28 \\
3366 \\
\pm 230 \\
5839 \\
\pm 115\end{array}$ & $\begin{array}{c}643 \\
\pm 55 \\
3427 \\
\pm 104 \\
6007 \\
\pm 31\end{array}$ & $\begin{array}{c}709 \\
\pm 81 \\
3340 \\
\pm 268 \\
5939 \\
\pm 243\end{array}$ & $\begin{array}{c}640 \\
\pm 22 \\
3323 \\
\pm 310 \\
5450 \\
\pm 508\end{array}$ & $\begin{array}{c}783 \\
\pm 279 \\
3293 \\
\pm 224 \\
5878 \\
\pm 128\end{array}$ \\
\hline
\end{tabular}

Tabelle 20

Sauerstoffgehalt im Belüftungsbecken [mg O/I]

\begin{tabular}{|c|c|c|c|c|c|c|c|}
\hline \multirow{3}{*}{$\begin{array}{l}\mathrm{O}_{2} \text {-Konz. } \\
\mathrm{BB} \\
\mathrm{mg} / \mathrm{l}\end{array}$} & \multirow{3}{*}{$\begin{array}{l}\text { Schlamm- } \\
\text { konz. BB } \\
\mathrm{mg} / \mathrm{l}\end{array}$} & \multirow{2}{*}{\multicolumn{3}{|c|}{$\begin{array}{l}\text { Mittlere Temperatur } \mathrm{BB}= \\
>13^{\circ} \mathrm{C} \\
\text { Hydraulische Raumbelastung } \\
\mathrm{m}^{3} / \mathrm{m}^{3} \text {. Tag }\end{array}$}} & \multirow{2}{*}{\multicolumn{3}{|c|}{$\begin{array}{l}\text { Mittlere Temperatur } \mathrm{BB}= \\
<11^{\circ} \mathrm{C} \\
\text { Hydraulische Raumbelastung } \\
\mathrm{m}^{3} / \mathrm{m}^{3} \cdot \text { Tag }\end{array}$}} \\
\hline & & & & & & & \\
\hline & & 58 & 29 & 9 & 58 & 29 & 9 \\
\hline 1 & $\begin{array}{r}600 \\
3300 \\
6000\end{array}$ & $\begin{array}{l}0,8 \pm 0,2 \\
1,7 \pm 0,9 \\
0,9 \pm 0,3\end{array}$ & $\begin{array}{l}2,0 \pm 0,8 \\
1,3 \pm 0,5 \\
0,5 \pm 0,2\end{array}$ & $\begin{array}{l}1,4 \pm 0,2 \\
0,9 \pm 0,2 \\
1,1 \pm 0,6\end{array}$ & $\begin{array}{l}1,6 \pm 1,0 \\
1,1 \pm 0,3 \\
1,9 \pm 0,9\end{array}$ & $\begin{array}{l}1,5 \pm 0,3 \\
1,0 \pm 0,4 \\
1,3 \pm 0,8\end{array}$ & $\begin{array}{l}1,2 \pm 0,6 \\
1,2 \pm 0,2 \\
1,1 \pm 0,3\end{array}$ \\
\hline 4 & $\begin{array}{r}600 \\
3300 \\
6000\end{array}$ & $\begin{array}{l}4,2 \pm 0,7 \\
3,0 \pm 0,5 \\
4,5 \pm 0,5\end{array}$ & $\begin{array}{l}4,3 \pm 0,7 \\
3,9 \pm 0,9 \\
4,0 \pm 0,3\end{array}$ & $\begin{array}{l}4,5 \pm 0,8 \\
3,9 \pm 0,5 \\
4,1 \pm 0,5\end{array}$ & $\begin{array}{l}4,0 \pm 0,8 \\
4,2 \pm 0,5 \\
5,0 \pm 0,9\end{array}$ & $\begin{array}{l}4,6 \pm 0,8 \\
4,2 \pm 0,4\end{array}$ & $\begin{array}{l}4,0 \pm 0,2 \\
4,5 \pm 0,5 \\
4,2 \pm 0,4\end{array}$ \\
\hline 7 & $\begin{array}{r}600 \\
3300 \\
6000\end{array}$ & $\begin{array}{l}6,8 \pm 0,1 \\
6,8 \pm 0,4 \\
6,9 \pm 0,3\end{array}$ & $\begin{array}{l}7,2 \pm 1,1 \\
6,7 \pm 0,4 \\
6,7 \pm 0,7\end{array}$ & $\begin{array}{l}6,1 \pm 0,5 \\
6,8 \pm 0,1 \\
6,7 \pm 0,6\end{array}$ & $\begin{array}{l}7,2 \pm 0,2 \\
6,5 \pm 0,6 \\
6,8 \pm 1,0\end{array}$ & $\begin{array}{l}7,0 \pm 0,5 \\
7,1 \pm 1,0 \\
6,9 \pm 0,4\end{array}$ & $\begin{array}{l}6,3 \pm 0,1 \\
7,0 \pm 0,3 \\
6,9 \pm 2,8\end{array}$ \\
\hline
\end{tabular}


Tabelle 21

Rücklaufschlammenge im Verhältnis zur Abwassermenge

\begin{tabular}{|c|c|c|c|c|c|c|c|}
\hline \multirow{2}{*}{$\begin{array}{l}\mathrm{O}_{2}-\text { Konz. } \\
\mathrm{BB} \\
\mathrm{mg} / 1\end{array}$} & \multirow{2}{*}{$\begin{array}{l}\text { Schlamm- } \\
\text { konz. BB } \\
\text { mg/l }\end{array}$} & \multicolumn{3}{|c|}{$\begin{array}{l}\text { Mittlere Temperatur } \mathrm{BB}= \\
>13^{\circ} \mathrm{C} \\
\text { Hydraulische Raumbelastung } \\
\mathrm{m}^{3} / \mathrm{m}^{3} \cdot \text { Tag }\end{array}$} & \multicolumn{3}{|c|}{$\begin{array}{l}\text { Mittlere Temperatur } \mathrm{BB}= \\
<11^{\circ} \mathrm{C} \\
\text { Hydraulische Raumbelastung } \\
\mathrm{m}^{3} / \mathrm{m}^{3} \text {. Tag }\end{array}$} \\
\hline & & 58 & 29 & 9 & 58 & 29 & 9 \\
\hline 1 & $\begin{array}{r}600 \\
3300 \\
6000\end{array}$ & $\begin{array}{l}1,2 \\
1,2 \\
1,92\end{array}$ & $\begin{array}{l}1,2 \\
1,2 \\
1,2\end{array}$ & $\begin{array}{l}0,96 \\
0,96 \\
0,72\end{array}$ & $\begin{array}{l}1,2 \\
0,96 \\
1,92\end{array}$ & $\begin{array}{l}0,96 \\
0,96 \\
1,92\end{array}$ & $\begin{array}{l}1,2 \\
1,2 \\
1,92\end{array}$ \\
\hline 4 & $\begin{array}{r}600 \\
3300 \\
6000\end{array}$ & $\begin{array}{l}1,2 \\
1,2 \\
1,44\end{array}$ & $\begin{array}{l}1,2 \\
0,81 \\
0,62\end{array}$ & $\begin{array}{l}1,2 \\
0,58 \\
0,48\end{array}$ & $\begin{array}{l}0,96 \\
0,96 \\
1,08\end{array}$ & 1,92 & $\begin{array}{l}1,2 \\
0,96 \\
1,44\end{array}$ \\
\hline 7 & $\begin{array}{r}600 \\
3300 \\
6000\end{array}$ & $\begin{array}{l}0,96 \\
0,72 \\
1,08\end{array}$ & $\begin{array}{l}0,96 \\
0,58 \\
0,96\end{array}$ & $\begin{array}{l}0,96 \\
0,96 \\
0,96\end{array}$ & $\begin{array}{l}0,72 \\
1,2 \\
1,08\end{array}$ & $\begin{array}{l}0,96 \\
1,2 \\
1,2\end{array}$ & $\begin{array}{l}2,16 \\
0,96 \\
1,68\end{array}$ \\
\hline
\end{tabular}

Tabelle 22

Raumbelastung mit org. $C \quad$ [kg org. $\left.\mathrm{C} / \mathrm{m}^{3}{ }_{B B} \cdot \mathrm{Tag}\right]$

\begin{tabular}{|c|c|c|c|c|c|c|c|}
\hline \multirow{2}{*}{$\begin{array}{l}\mathrm{O}_{2}-\text { Konz. } \\
\mathrm{BB} \\
\mathrm{mg} / \mathrm{l}\end{array}$} & \multirow{2}{*}{$\begin{array}{l}\text { Schlamm- } \\
\text { konz. BB } \\
\mathrm{mg} / \mathrm{l}\end{array}$} & \multicolumn{3}{|c|}{$\begin{array}{l}\text { Mittlere Temperatur } \mathrm{BB}= \\
>13^{\circ} \mathrm{C} \\
\text { Hydraulische Raumbelastung } \\
\mathrm{m}^{3} / \mathrm{m}^{3} \text {. Tag }\end{array}$} & \multicolumn{3}{|c|}{$\begin{array}{l}\text { Mittlere Temperatur } \mathrm{BB}= \\
<11^{\circ} \mathrm{C} \\
\text { Hydraulische Raumbelastung } \\
\mathrm{m}^{3} / \mathrm{m}^{3} \cdot \text { Tag }\end{array}$} \\
\hline & & 58 & 29 & 9 & 58 & 29 & 9 \\
\hline 1 & $\begin{array}{r}600 \\
3300 \\
6000\end{array}$ & $\begin{array}{l}3,69 \\
3,05 \\
3,43\end{array}$ & $\begin{array}{l}1,48 \\
1,86 \\
1,54\end{array}$ & $\begin{array}{l}0,43 \\
0,57 \\
0,58\end{array}$ & $\begin{array}{l}3,72 \\
2,79 \\
4,43\end{array}$ & $\begin{array}{l}1,59 \\
1,36 \\
1,59\end{array}$ & $\begin{array}{l}0,58 \\
0,49 \\
0,44\end{array}$ \\
\hline 4 & $\begin{array}{r}600 \\
3300 \\
6000\end{array}$ & $\begin{array}{l}4,11 \\
3,06 \\
3,67\end{array}$ & $\begin{array}{l}2,07 \\
1,83 \\
1,53\end{array}$ & $\begin{array}{l}0,54 \\
0,55 \\
0,66\end{array}$ & $\begin{array}{l}3,56 \\
2,69 \\
2,34\end{array}$ & 1,59 & $\begin{array}{l}0,56 \\
0,57 \\
0,60\end{array}$ \\
\hline 7 & $\begin{array}{r}600 \\
3300 \\
6000\end{array}$ & $\begin{array}{l}3,05 \\
3,45 \\
3,45\end{array}$ & $\begin{array}{l}2,12 \\
1,71 \\
1,72\end{array}$ & $\begin{array}{l}0,49 \\
0,48 \\
0,66\end{array}$ & $\begin{array}{l}3,77 \\
3,44 \\
3,12\end{array}$ & $\begin{array}{l}1,79 \\
1,85 \\
1,74\end{array}$ & $\begin{array}{l}0,37 \\
0,43 \\
0,70\end{array}$ \\
\hline
\end{tabular}


Tabelle 23

Raumbelastung mit $B S B_{5} \quad\left[\mathrm{~kg} \mathrm{BSB} \mathrm{BS}_{5} / \mathrm{m}^{3} B B \cdot \mathrm{Tag}\right]$

\begin{tabular}{|c|c|c|c|}
\hline $\begin{array}{l}\mathrm{O}_{2} \text {-Konz. } \\
\mathrm{BB}\end{array}$ & $\begin{array}{l}\text { Schlamm- } \\
\text { konz. BB }\end{array}$ & $\begin{array}{l}\text { Mittlere Temperatur } \mathrm{BB}= \\
>13^{\circ} \mathrm{C} \\
\text { Hydraulische Raumbelastung } \\
\mathrm{m}^{3} / \mathrm{m}^{3} \cdot \text { Tag }\end{array}$ & $\begin{array}{l}\text { Mittlere Temperatur } \mathrm{BB}= \\
<11^{\circ} \mathrm{C} \\
\text { Hydraulische Raumbelastung } \\
\mathrm{m}^{3} / \mathrm{m}^{3} \text {. Tag }\end{array}$ \\
\hline
\end{tabular}

\begin{tabular}{lrllllll}
$\mathrm{mg} / \mathrm{l}$ & $\mathrm{mg} / \mathrm{l}$ & 58 & 29 & 9 & 58 & 29 & 9 \\
\hline 1 & 600 & 6,11 & 2,65 & 0,79 & 6,39 & 3,04 & 1,0 \\
& 3300 & 5,44 & 2,71 & 1,35 & 5,25 & 3,30 & 0,95 \\
& 6000 & 6,96 & 2,82 & 0,85 & 7,44 & 3,97 & 0,84 \\
4 & 600 & 6,66 & 3,35 & 1,07 & 6,65 & 3,76 & 1,06 \\
& 3300 & 5,59 & 4,31 & 1,12 & 6,53 & & 1,06 \\
& 6000 & 5,35 & 3,95 & 1,07 & 6,68 & 3,96 & 1,10 \\
7 & 600 & 7,84 & 4,10 & 0,89 & 6,94 & 3,35 & 1,06 \\
& 3300 & 5,75 & 3,48 & 1,25 & 6,51 & 3,18 & 1,05 \\
& 6000 & 5,23 & 2,87 & 1,28 & 5,98 & 3,29 & 1,18 \\
\hline
\end{tabular}

Tabelle 24

Schlammbelastung [kg org. C/kg Trockensubstanz. Tag]

\begin{tabular}{|c|c|c|c|c|c|c|c|}
\hline \multirow[t]{2}{*}{$\begin{array}{l}\mathrm{O}_{2}-\text { Konz. } \\
\mathrm{BB}\end{array}$} & \multirow{2}{*}{$\begin{array}{l}\text { Schlamm- } \\
\text { konz. BB } \\
\mathrm{mg} / 1\end{array}$} & \multicolumn{3}{|c|}{$\begin{array}{l}\text { Mittlere Temperatur } \mathrm{BB}= \\
>13^{\circ} \mathrm{C} \\
\text { Hydraulische Raumbelastung } \\
\mathrm{m}^{3} / \mathrm{m}^{3} \cdot \text { Tag }\end{array}$} & \multicolumn{3}{|c|}{$\begin{array}{l}\text { Mittlere Temperatur } \mathrm{BB}= \\
<11^{\circ} \mathrm{C} \\
\text { Hydraulische Raumbelastung } \\
\mathrm{m}^{3} / \mathrm{m}^{3} \cdot \text { Tag }\end{array}$} \\
\hline & & 58 & 29 & 9 & 58 & 29 & 9 \\
\hline 1 & $\begin{array}{r}600 \\
3300 \\
6000\end{array}$ & $\begin{array}{l}5,74 \\
0,94 \\
0,57\end{array}$ & $\begin{array}{l}2,18 \\
0,55 \\
0,25\end{array}$ & $\begin{array}{l}0,67 \\
0,17 \\
0,09\end{array}$ & $\begin{array}{l}5,63 \\
0,82 \\
0,77\end{array}$ & $\begin{array}{l}2,65 \\
0,41 \\
0,26\end{array}$ & $\begin{array}{l}0,88 \\
0,15 \\
0,07\end{array}$ \\
\hline 4 & $\begin{array}{r}600 \\
3300 \\
6000\end{array}$ & $\begin{array}{l}5,86 \\
0,92 \\
0,61\end{array}$ & $\begin{array}{l}3,09 \\
0,54 \\
0,26\end{array}$ & $\begin{array}{l}0,84 \\
0,17 \\
0,11\end{array}$ & $\begin{array}{l}5,07 \\
0,78 \\
0,38\end{array}$ & 3,28 & $\begin{array}{l}0,83 \\
0,17 \\
0,10\end{array}$ \\
\hline 7 & $\begin{array}{r}600 \\
3300 \\
6000\end{array}$ & $\begin{array}{l}4,53 \\
1,01 \\
0,57\end{array}$ & $\begin{array}{l}2,99 \\
0,51 \\
0,29\end{array}$ & $\begin{array}{l}0,76 \\
0,14 \\
0,11\end{array}$ & $\begin{array}{l}5,32 \\
1,03 \\
0,52\end{array}$ & $\begin{array}{l}2,80 \\
0,56 \\
0,32\end{array}$ & $\begin{array}{l}0,47 \\
0,13 \\
0,12\end{array}$ \\
\hline
\end{tabular}


Tabelle 25

Schlammbelastung $\quad[\mathrm{kg} \mathrm{BSB} / \mathrm{kg}$ Trockensubstanz $\cdot$ Tag]

\begin{tabular}{|c|c|c|c|c|c|c|c|}
\hline \multirow{2}{*}{$\begin{array}{l}\mathrm{O}_{2}-\text { Konz. } \\
\mathrm{BB}^{-} \\
\mathrm{mg} / \mathrm{l}\end{array}$} & \multirow{2}{*}{$\begin{array}{l}\text { Schlamm- } \\
\text { konz. BB } \\
\mathrm{mg} / \mathrm{l}\end{array}$} & \multicolumn{3}{|c|}{$\begin{array}{l}\text { Mittlere Temperatur } \mathrm{BB}= \\
>13^{\circ} \mathrm{C} \\
\mathrm{Hydraulische} \text { Raumbelastung } \\
\mathrm{m}^{3} / \mathrm{m}^{3} \cdot \text { Tag }\end{array}$} & \multicolumn{3}{|c|}{$\begin{array}{l}\text { Mittlere Temperatur } \mathrm{BB}= \\
<11^{\circ} \mathrm{C} \\
\text { Hydraulische Raumbelastung } \\
\mathrm{m}^{3} / \mathrm{m}^{3} \text {. Tag }\end{array}$} \\
\hline & & 58 & 29 & 9 & 58 & 29 & 9 \\
\hline 1 & $\begin{array}{r}600 \\
3300 \\
6000\end{array}$ & $\begin{array}{l}9,49 \\
1,67 \\
1,17\end{array}$ & $\begin{array}{l}3,90 \\
0,80 \\
0,46\end{array}$ & $\begin{array}{l}1,23 \\
0,39 \\
0,14\end{array}$ & $\begin{array}{l}9,68 \\
1,55 \\
1,29\end{array}$ & $\begin{array}{l}5,06 \\
0,99 \\
0,65\end{array}$ & $\begin{array}{l}1,52 \\
0,29 \\
0,14\end{array}$ \\
\hline 4 & $\begin{array}{r}600 \\
3300 \\
6000\end{array}$ & $\begin{array}{l}9,49 \\
1,80 \\
0,89\end{array}$ & $\begin{array}{l}5,0 \\
1,31 \\
0,67\end{array}$ & $\begin{array}{l}1,67 \\
0,35 \\
0,16\end{array}$ & $\begin{array}{l}9,45 \\
1,92 \\
1,08\end{array}$ & 0,64 & $\begin{array}{l}1,56 \\
0,31 \\
0,18\end{array}$ \\
\hline 7 & $\begin{array}{r}600 \\
3300 \\
6000\end{array}$ & $\begin{array}{l}11,6 \\
1,68 \\
0,87\end{array}$ & $\begin{array}{l}5,78 \\
1,03 \\
0,49\end{array}$ & $\begin{array}{l}1,40 \\
0,36 \\
0,21\end{array}$ & $\begin{array}{l}9,80 \\
1,95 \\
0,99\end{array}$ & $\begin{array}{l}5,23 \\
0,95 \\
0,60\end{array}$ & $\begin{array}{l}1,36 \\
0,32 \\
0,20\end{array}$ \\
\hline
\end{tabular}

Tabelle 26

Reinigungseffeht $\quad[\%$ org. $\mathrm{C}]$

\begin{tabular}{|c|c|c|c|c|c|c|c|}
\hline \multirow{2}{*}{$\begin{array}{l}\mathrm{O}_{2} \text {-Konz. } \\
\mathrm{BB} \\
\mathrm{mg} / 1\end{array}$} & \multirow{2}{*}{$\begin{array}{l}\text { Schlamm- } \\
\text { konz. BB } \\
\mathrm{mg} / 1\end{array}$} & \multicolumn{3}{|c|}{$\begin{array}{l}\text { Mittlere Temperatur } \mathrm{BB}= \\
>13^{\circ} \mathrm{C} \\
\text { Hydraulische Raumbelastung } \\
\mathrm{m}^{3} / \mathrm{m}^{3} \cdot \text { Tag }\end{array}$} & \multicolumn{3}{|c|}{$\begin{array}{l}\text { Mittlere Temperatur } \mathrm{BB}= \\
<11^{\circ} \mathrm{C} \\
\text { Hydraulische Raumbelastung } \\
\mathrm{m}^{3} / \mathrm{m}^{3} \cdot \text { Tag }\end{array}$} \\
\hline & & 58 & 29 & 9 & 58 & 29 & 9 \\
\hline 1 & $\begin{array}{r}600 \\
3300 \\
6000\end{array}$ & $\begin{array}{l}49,0 \\
71,4 \\
74,5\end{array}$ & $\begin{array}{l}63,5 \\
79,5 \\
71,0\end{array}$ & $\begin{array}{l}64,0 \\
85,4 \\
85,0\end{array}$ & $\begin{array}{l}30,4 \\
64,5 \\
67\end{array}$ & $\begin{array}{l}46,2 \\
72,4 \\
71,7\end{array}$ & $\begin{array}{l}57,7 \\
81,3 \\
78,5\end{array}$ \\
\hline 4 & $\begin{array}{r}600 \\
3300 \\
6000\end{array}$ & $\begin{array}{l}70,0 \\
70,0 \\
75,8\end{array}$ & $\begin{array}{l}75,4 \\
81,5 \\
83,7\end{array}$ & $\begin{array}{l}66,7 \\
80,4 \\
86,4\end{array}$ & $\begin{array}{l}38,8 \\
59,5 \\
65,8\end{array}$ & 53,2 & $\begin{array}{l}60,0 \\
79,1 \\
79,0\end{array}$ \\
\hline 7 & $\begin{array}{r}600 \\
3300 \\
6000\end{array}$ & $\begin{array}{l}61,8 \\
75,4 \\
78,9\end{array}$ & $\begin{array}{l}65,4 \\
78,0 \\
79,8\end{array}$ & $\begin{array}{l}70,4 \\
79,0 \\
83,6\end{array}$ & $\begin{array}{l}36,1 \\
61,6 \\
72,2\end{array}$ & $\begin{array}{l}48,6 \\
63,7 \\
67,7\end{array}$ & $\begin{array}{l}63,6 \\
73,4 \\
78,6\end{array}$ \\
\hline
\end{tabular}


Tabelle 27

Reinigungseffekt $[\%$ org. $\mathrm{N}]$

\begin{tabular}{|c|c|c|c|c|c|c|c|}
\hline \multirow{2}{*}{$\begin{array}{l}\mathrm{O}_{2}-\text { Konz. } \\
\mathrm{BB} \\
\mathrm{mg} / 1\end{array}$} & \multirow{2}{*}{$\begin{array}{l}\text { Schlamm- } \\
\text { konz. BB } \\
\mathrm{mg} / 1\end{array}$} & \multicolumn{3}{|c|}{$\begin{array}{l}\text { Mittlere Temperatur } \mathrm{BB}= \\
>13^{\circ} \mathrm{C} \\
\text { Hydraulische Raumbelastung } \\
\mathrm{m}^{3} / \mathrm{m}^{3} \text {. Tag }\end{array}$} & \multicolumn{3}{|c|}{$\begin{array}{l}\text { Mittlere Temperatur } \mathrm{BB}= \\
<11^{\circ} \mathrm{C} \\
\text { Hydraulische Raumbelastung } \\
\mathrm{m}^{3} / \mathrm{m}^{3} \text {. Tag }\end{array}$} \\
\hline & & 58 & 29 & 9 & 58 & 29 & 9 \\
\hline 1 & $\begin{array}{r}600 \\
3300 \\
6000\end{array}$ & $\begin{array}{l}42,4 \\
68,4 \\
73,0\end{array}$ & $\begin{array}{l}54,6 \\
70,9 \\
71,0\end{array}$ & $\begin{array}{l}55,5 \\
85,6 \\
87,0\end{array}$ & $\begin{array}{l}46,7 \\
60,6 \\
58,4\end{array}$ & $\begin{array}{l}45,1 \\
68,2 \\
70,3\end{array}$ & $\begin{array}{l}61,3 \\
75,4 \\
88,2\end{array}$ \\
\hline 4 & $\begin{array}{r}600 \\
3300 \\
6000\end{array}$ & $\begin{array}{l}61,0 \\
65,1 \\
70,7\end{array}$ & $\begin{array}{l}64,4 \\
75,6 \\
79,5\end{array}$ & $\begin{array}{l}64,7 \\
75,5 \\
86,2\end{array}$ & $\begin{array}{l}40,3 \\
54,4 \\
66,0\end{array}$ & $\begin{array}{l}36,9 \\
71,3\end{array}$ & $\begin{array}{l}56,8 \\
75,6 \\
87,0\end{array}$ \\
\hline 7 & $\begin{array}{r}600 \\
3300 \\
6000\end{array}$ & $\begin{array}{l}47,5 \\
80,1 \\
79,3\end{array}$ & $\begin{array}{l}48,1 \\
66,0 \\
86,0\end{array}$ & $\begin{array}{l}69,4 \\
80,6 \\
83,5\end{array}$ & $\begin{array}{l}23,4 \\
64,4 \\
70,6\end{array}$ & $\begin{array}{l}49,4 \\
62,1 \\
74,2\end{array}$ & $\begin{array}{l}62,1 \\
74,2 \\
74,5\end{array}$ \\
\hline
\end{tabular}

Tabelle 28

Reinigungseffekt $\left[\begin{array}{lll}\% & \mathrm{BSB}_{5}\end{array}\right]$

$\mathrm{O}_{2}$-Konz. Schlamm- Mittlere Temperatur $\mathrm{BB}=$ Mittlere Temperatur $\mathrm{BB}=$ $\mathrm{BB} \quad$ konz. BB $>13^{\circ} \mathrm{C}<11^{\circ} \mathrm{C}$

Hydraulische Raumbelastung Hydraulische Raumbelastung $\mathrm{m}^{3} / \mathrm{m}^{3}$. Tag $\mathrm{m}^{3} / \mathrm{m}^{3}$. Tag

\begin{tabular}{|c|c|c|c|c|c|c|c|}
\hline $\mathrm{mg} / \mathrm{l}$ & $\mathrm{mg} / \mathrm{l}$ & 58 & 29 & 9 & 58 & 29 & 9 \\
\hline 1 & $\begin{array}{r}600 \\
3300 \\
6000\end{array}$ & $\begin{array}{l}56,2 \\
87,5 \\
85,4\end{array}$ & $\begin{array}{l}79,0 \\
89,4 \\
84,0\end{array}$ & $\begin{array}{l}80,0 \\
94,0 \\
90,5\end{array}$ & $\begin{array}{l}40,9 \\
81,4 \\
82\end{array}$ & $\begin{array}{l}50,5 \\
84,1 \\
91,1\end{array}$ & $\begin{array}{l}82,7 \\
92,4 \\
92,3\end{array}$ \\
\hline 4 & $\begin{array}{r}600 \\
3300 \\
6000\end{array}$ & $\begin{array}{l}75,8 \\
80,5 \\
87,2\end{array}$ & $\begin{array}{l}82,7 \\
92,6 \\
89,2\end{array}$ & $\begin{array}{l}90,7 \\
91,0 \\
93,2\end{array}$ & $\begin{array}{l}52,2 \\
86,0 \\
87,0\end{array}$ & $\begin{array}{l}57,4 \\
91,8\end{array}$ & $\begin{array}{l}76,9 \\
93,9 \\
94,9\end{array}$ \\
\hline 7 & $\begin{array}{r}600 \\
3300 \\
6000\end{array}$ & $\begin{array}{l}73,2 \\
91,0 \\
83,7\end{array}$ & $\begin{array}{l}76,2 \\
88,5 \\
93,0\end{array}$ & $\begin{array}{l}87,0 \\
88,5 \\
90,5\end{array}$ & $\begin{array}{l}57,9 \\
79,5 \\
83,8\end{array}$ & $\begin{array}{l}63,5 \\
83,6 \\
80,3\end{array}$ & $\begin{array}{l}78,2 \\
92,0 \\
86,8\end{array}$ \\
\hline
\end{tabular}


Tabelle 29 Überschußschlammproduktion [g Trockensubstan $z / \mathrm{m}^{3}$ Abwasser] (inklusive Schwebe-
stoffe im NKB-Abfluss)

\begin{tabular}{|c|c|c|c|c|c|c|c|}
\hline \multirow{2}{*}{$\begin{array}{l}\mathrm{O}_{2}-\mathrm{Konz} . \\
\mathrm{BB} \\
\mathrm{mg} / \mathrm{l}\end{array}$} & \multirow{2}{*}{$\begin{array}{l}\text { Schlamm- } \\
\text { konz. BB } \\
\mathrm{mg} / \mathrm{l}\end{array}$} & \multicolumn{3}{|c|}{$\begin{array}{l}\text { Mittlere Temperatur } \mathrm{BB}= \\
>13^{\circ} \mathrm{C} \\
\text { Hydraulische Raumbelastung } \\
\mathrm{m}^{3} / \mathrm{m}^{3} \cdot \text { Tag }\end{array}$} & \multicolumn{3}{|c|}{$\begin{array}{l}\text { Mittlere Temperatur } \mathrm{BB}= \\
<11^{\circ} \mathrm{C} \\
\text { Hydraulische Raumbelastung } \\
\mathrm{m}^{3} / \mathrm{m}^{3} \cdot \text { Tag }\end{array}$} \\
\hline & & 58 & 29 & 9 & 58 & 29 & 9 \\
\hline 1 & $\begin{array}{r}600 \\
3300 \\
6000\end{array}$ & $\begin{array}{l}103 \\
125 \\
141\end{array}$ & $\begin{array}{r}94 \\
146 \\
92\end{array}$ & $\begin{array}{r}113 \\
86 \\
110\end{array}$ & $\begin{array}{r}95 \\
118 \\
115\end{array}$ & $\begin{array}{l}115 \\
146 \\
139\end{array}$ & $\begin{array}{r}118 \\
126 \\
92\end{array}$ \\
\hline 4 & $\begin{array}{r}600 \\
3300 \\
6000\end{array}$ & $\begin{array}{r}105 \\
123 \\
95\end{array}$ & $\begin{array}{r}103 \\
90 \\
71\end{array}$ & $\begin{array}{r}118 \\
77 \\
110\end{array}$ & $\begin{array}{l}103 \\
125 \\
106\end{array}$ & 178 & $\begin{array}{r}137 \\
135 \\
80\end{array}$ \\
\hline 7 & $\begin{array}{r}600 \\
3300 \\
6000\end{array}$ & $\begin{array}{r}72 \\
147 \\
58\end{array}$ & $\begin{array}{r}100 \\
84 \\
98\end{array}$ & $\begin{array}{r}100 \\
68 \\
131\end{array}$ & $\begin{array}{r}95 \\
135 \\
110\end{array}$ & $\begin{array}{r}97 \\
100 \\
88\end{array}$ & $\begin{array}{r}128 \\
123 \\
65\end{array}$ \\
\hline
\end{tabular}

Tabelle 30

Uberschußschlammproduktion [g org. $\mathrm{C} / \mathrm{m}^{3}$ Abwasser] (inklusive Schwebestoffe im
NKB-Abfluss)

\begin{tabular}{|c|c|c|c|c|c|c|c|}
\hline \multirow{2}{*}{$\begin{array}{l}\mathrm{O}_{2}-\text { Kon } z . \\
\mathrm{BB} \\
\mathrm{mg} / 1\end{array}$} & \multirow{2}{*}{$\begin{array}{l}\text { Schlamm- } \\
\text { konz. BB } \\
\mathrm{mg} / \mathrm{I}\end{array}$} & \multicolumn{3}{|c|}{$\begin{array}{l}\text { Mittlere Temperatur } \mathrm{BB}= \\
\mathrm{P}^{\circ} 3^{\circ} \mathrm{C} \\
\text { Hydraulische Raumbelastung } \\
\mathrm{m}^{3} / \mathrm{m}^{3} \cdot \text { Tag }\end{array}$} & \multicolumn{3}{|c|}{$\begin{array}{l}\text { Mittlere Temperatur } \mathrm{BB}= \\
<11^{\circ} \mathrm{C} \\
\text { Hydraulische Raumbelastung } \\
\mathrm{m}^{3} / \mathrm{m}^{3} \cdot \text { Tag }\end{array}$} \\
\hline & & 58 & 29 & 9 & 58 & 29 & 9 \\
\hline 1 & $\begin{array}{r}600 \\
3300 \\
6000\end{array}$ & $\begin{array}{l}31,3 \\
41,4 \\
49,2\end{array}$ & $\begin{array}{l}31,0 \\
51,4 \\
31,7\end{array}$ & $\begin{array}{l}35,8 \\
31,0 \\
36,7\end{array}$ & $\begin{array}{l}30,5 \\
40,4 \\
41,2\end{array}$ & $\begin{array}{l}39,7 \\
49,9 \\
47,6\end{array}$ & $\begin{array}{l}38,3 \\
43,5 \\
31,5\end{array}$ \\
\hline 4 & $\begin{array}{r}600 \\
3300 \\
6000\end{array}$ & $\begin{array}{l}36,4 \\
43,1 \\
32,2\end{array}$ & $\begin{array}{l}37,0 \\
32,8 \\
24,1\end{array}$ & $\begin{array}{l}43,5 \\
26,4 \\
33,6\end{array}$ & $\begin{array}{l}36,0 \\
43,2 \\
36,3\end{array}$ & 39,7 & $\begin{array}{l}46,9 \\
45,7 \\
27,3\end{array}$ \\
\hline 7 & $\begin{array}{r}600 \\
3300 \\
6000\end{array}$ & $\begin{array}{l}25,1 \\
57,0 \\
20,6\end{array}$ & $\begin{array}{l}37,0 \\
29,4 \\
32,1\end{array}$ & $\begin{array}{l}36,6 \\
22,6 \\
47,2\end{array}$ & $\begin{array}{l}32,7 \\
46,3 \\
38,1\end{array}$ & $\begin{array}{l}32,4 \\
30,9 \\
30,0\end{array}$ & $\begin{array}{l}43,8 \\
42,1 \\
20,6\end{array}$ \\
\hline
\end{tabular}


Tabelle 31

Mittelwerte und Streunngen der Schwebestoffkonzentration [mg Trockensubstanz/l]

\begin{tabular}{|c|c|c|c|c|c|c|c|c|c|c|c|c|c|}
\hline \multirow{2}{*}{$\begin{array}{l}\mathrm{O}_{2}-\text { Konz. } \\
\mathrm{BB} \\
\mathrm{mg} / 1\end{array}$} & \multirow{2}{*}{$\begin{array}{l}\text { Schlamm- } \\
\text { konz. BB } \\
\mathrm{mg} / 1\end{array}$} & \multicolumn{6}{|c|}{$\begin{array}{l}\text { Mittlere Temperatur } \mathrm{BB}=>13^{\circ} \mathrm{C} \\
\text { Hydraulische Raumbelastung } \mathrm{m}^{3} / \mathrm{m}^{3} \cdot \text { Tag }\end{array}$} & \multicolumn{6}{|c|}{$\begin{array}{l}\text { Mittlere Temperatur } \mathrm{BB}=<11^{\circ} \mathrm{C} \\
\text { Hydraulische Raumbelastung } \mathrm{m}^{3} / \mathrm{m}^{3} \cdot \text { Tag }\end{array}$} \\
\hline & & $\begin{array}{l}58 \\
Z\end{array}$ & A & $\begin{array}{l}29 \\
Z\end{array}$ & $\mathrm{~A}$ & $\begin{array}{l}9 \\
Z\end{array}$ & $\mathrm{~A}$ & $\begin{array}{l}58 \\
Z\end{array}$ & A & 29 & $\mathrm{~A}$ & $\begin{array}{l}9 \\
Z\end{array}$ & A \\
\hline 1 & $\begin{array}{r}600 \\
3300 \\
6000\end{array}$ & $\begin{array}{c}109 \\
\pm 15 \\
90 \\
\pm 9 \\
104 \\
\pm 16\end{array}$ & $\begin{array}{l}35 \\
\pm 7 \\
22 \\
\pm 7 \\
29 \\
\pm 17\end{array}$ & $\begin{array}{c}90 \\
\pm 9 \\
119 \\
\pm 33 \\
103 \\
\pm 37\end{array}$ & $\begin{array}{c}29 \\
\pm 6 \\
19 \\
\pm 4 \\
15 \\
\pm 6\end{array}$ & $\begin{array}{c}83 \\
\pm 10 \\
110 \\
\pm 9 \\
119 \\
\pm 33\end{array}$ & $\begin{array}{c}27 \\
\pm 4 \\
14 \\
\pm 5 \\
14 \\
\pm 4\end{array}$ & $\begin{array}{c}91 \\
\pm 17 \\
178 \\
\pm 51 \\
145 \\
\pm 51\end{array}$ & $\begin{array}{l}55 \\
\pm 12 \\
41 \\
\pm 7 \\
23 \\
\pm 10\end{array}$ & $\begin{array}{c}101 \\
\pm 15 \\
240 \\
\pm 95 \\
131 \\
\pm 19\end{array}$ & $\begin{array}{l}49 \\
\pm 18 \\
75 \\
\pm 39 \\
26 \\
\pm 1\end{array}$ & $\begin{array}{c}91 \\
\pm 17 \\
101 \\
\pm 15 \\
178 \\
\pm 51\end{array}$ & $\begin{array}{l}39 \\
\pm 15 \\
22 \\
\pm 16 \\
27 \\
\pm 13\end{array}$ \\
\hline 4 & $\begin{array}{r}600 \\
3300\end{array}$ & $\begin{array}{c}153 \\
\pm 40 \\
103 \\
\pm 37 \\
119 \\
\pm 33\end{array}$ & $\begin{array}{l}36 \\
\pm 5 \\
18 \\
\pm 5 \\
20 \\
\pm 5\end{array}$ & $\begin{array}{c}153 \\
\pm 40 \\
110 \\
\pm 9 \\
94 \\
\pm 9\end{array}$ & $\begin{array}{c}31 \\
\pm 4 \\
20 \\
\pm 3 \\
13 \\
\pm 4\end{array}$ & $\begin{array}{c}85 \\
\pm 20 \\
104 \\
\pm 16 \\
153 \\
\pm 40\end{array}$ & $\begin{array}{c}19 \\
\pm 6 \\
19 \\
\pm 6 \\
15 \\
\pm 4\end{array}$ & $\begin{array}{c}126 \\
\pm 42 \\
240 \\
\pm 95 \\
138 \\
\pm 50\end{array}$ & $\begin{array}{l}49 \\
\pm 9 \\
50 \\
\pm 25 \\
49 \\
\pm 26\end{array}$ & $\begin{array}{c}145 \\
\pm 51 \\
. \\
131 \\
\pm 19\end{array}$ & $\begin{array}{l}58 \\
\pm 11\end{array}$ & $\begin{array}{c}106 \\
\pm 14 \\
126 \\
\pm 42 \\
105 \\
\pm 24\end{array}$ & $\begin{array}{l}37 \\
\pm 5 \\
24 \\
\pm 10 \\
12 \\
\pm 9\end{array}$ \\
\hline 7 & 3300 & $\begin{array}{c}93 \\
\pm 9 \\
177 \\
\pm 40 \\
149 \\
\pm 39\end{array}$ & $\begin{array}{l}31 \\
\pm 4 \\
61 \\
\pm 10 \\
41 \\
\pm 6\end{array}$ & $\begin{array}{c}114 \\
\pm 11 \\
104 \\
\pm 16 \\
203 \\
\pm 89\end{array}$ & $\begin{array}{c}37 \\
\pm 8 \\
17 \\
\pm 4 \\
(70) \\
( \pm 29)\end{array}$ & $\begin{array}{c}103 \\
\pm 37 \\
93 \\
\pm 9 \\
114 \\
\pm 11\end{array}$ & $\begin{array}{c}14 \\
\pm 5 \\
16 \\
\pm 4 \\
19 \\
\pm 8\end{array}$ & $\begin{array}{c}105 \\
\pm 24 \\
106 \\
\pm 14 \\
101 \\
\pm 15\end{array}$ & $\begin{array}{l}56 \\
\pm 17 \\
36 \\
\pm 8 \\
23 \\
\pm 6\end{array}$ & $\begin{array}{c}126 \\
\pm 42 \\
91 \\
\pm 17 \\
106 \\
\pm 14\end{array}$ & $\begin{array}{l}43 \\
\pm 13 \\
27 \\
\pm 8 \\
23 \\
\pm 8\end{array}$ & $\begin{array}{c}138 \\
\pm 49 \\
240 \\
\pm 95 \\
145 \\
\pm 51\end{array}$ & $\begin{array}{c}55 \\
\pm 13 \\
58 \\
\pm 33 \\
23 \\
\pm 29\end{array}$ \\
\hline
\end{tabular}

$Z=$ Belüftungsbeckenzufluss

$A=$ Nachklärbeckenabfluss 
Tabelle 32

Mittelwerte und Streunngen des organischen Kohlenstoffs [mg org. $\mathrm{C} / 1]$

\begin{tabular}{|c|c|c|c|c|c|c|c|c|c|c|c|c|c|}
\hline \multirow{2}{*}{$\begin{array}{l}\mathrm{O}_{2}-\text { Konz. } \\
\mathrm{BB} \\
\mathrm{mg} / \mathrm{l}\end{array}$} & \multirow{2}{*}{$\begin{array}{l}\text { Schlamm- } \\
\text { konz. BB } \\
\text { mg/l }\end{array}$} & \multicolumn{6}{|c|}{$\begin{array}{l}\text { Mittlere Temperatur } \mathrm{BB}=>13^{\circ} \mathrm{C} \\
\text { Hydraulische Raumbelastung } \mathrm{m}^{3} / \mathrm{m}^{3} \cdot \text { Tag }\end{array}$} & \multicolumn{6}{|c|}{$\begin{array}{l}\text { Mittlere Temperatur BB }=<11^{\circ} \mathrm{C} \\
\text { Hydraulische Raumbelastung } \mathrm{m}^{3} / \mathrm{m}^{3} \cdot \text { Tag }\end{array}$} \\
\hline & & $\begin{array}{l}58 \\
Z\end{array}$ & A & $\begin{array}{l}29 \\
Z\end{array}$ & A & $\begin{array}{l}9 \\
Z\end{array}$ & A & $\begin{array}{l}58 \\
Z\end{array}$ & $\mathrm{~A}$ & $\begin{array}{l}29 \\
Z\end{array}$ & A & $\begin{array}{l}9 \\
Z\end{array}$ & A \\
\hline 1 & 6000 & $\begin{array}{c}63,4 \\
\pm 5,7 \\
53,0 \\
\pm 7,2 \\
60,1 \\
\pm 12,9\end{array}$ & $\begin{array}{c}32,4 \\
\pm 3,6 \\
15,2 \\
\pm 3,1 \\
15,4 \\
\pm 1,9\end{array}$ & $\begin{array}{c}53,0 \\
\pm 7,2 \\
64,6 \\
\pm 11,3 \\
53,2 \\
\pm 6,0\end{array}$ & $\begin{array}{c}19,4 \\
\pm 3,0 \\
13,2 \\
\pm 0,9 \\
15,4 \\
\pm 2,2\end{array}$ & $\begin{array}{c}48,9 \\
\pm 6,1 \\
63,4 \\
\pm 8,3 \\
64,6 \\
\pm 11,3\end{array}$ & $\begin{array}{c}17,6 \\
\pm 2,7 \\
9,2 \\
\pm 0,4 \\
9,7 \\
\pm 0,8\end{array}$ & $\begin{array}{c}64,0 \\
\pm 12,4 \\
48,4 \\
\pm 9,0 \\
76,7 \\
\pm 13,5\end{array}$ & $\begin{array}{c}39,5 \\
\pm 5,7 \\
17,2 \\
\pm 4,1 \\
24,6 \\
\pm 6,0\end{array}$ & $\begin{array}{c}54,7 \\
\pm 10,3 \\
46,6 \\
\pm 9.1 \\
54,2 \\
\pm 8,2\end{array}$ & $\begin{array}{c}29,1 \\
\pm 7,5 \\
12,9 \\
\pm 1,7 \\
15,3 \\
\pm 1,6\end{array}$ & $\begin{array}{c}64,0 \\
\pm 12,4 \\
54,7 \\
\pm 10,3 \\
48,4 \\
\pm 9,0\end{array}$ & $\begin{array}{c}27,1 \\
\pm 6,2 \\
10,3 \\
\pm 3,5 \\
10,4 \\
\pm 1,8\end{array}$ \\
\hline 4 & 6000 & $\begin{array}{c}71,6 \\
\pm 14,1 \\
53,2 \\
\pm 6,0 \\
64,6 \\
\pm 11,3\end{array}$ & $\begin{array}{c}21,4 \\
\pm 2,2 \\
16,0 \\
\pm 2,6 \\
15,6 \\
\pm 1,3\end{array}$ & $\begin{array}{c}71,6 \\
\pm 14,1 \\
63,4 \\
18,3 \\
53,5 \\
\pm 5,3\end{array}$ & $\begin{array}{c}17,7 \\
\pm 2,0 \\
11,8 \\
\pm 1,7 \\
8,9 \\
\pm 0,8\end{array}$ & $\begin{array}{c}59,3 \\
\pm 8,6 \\
60,1 \\
\pm 12,9 \\
71,6 \\
\pm 14,1\end{array}$ & $\begin{array}{c}19,8 \\
\pm 3,7 \\
11,8 \\
\pm 3,4 \\
9,8 \\
\pm 2,9\end{array}$ & $\begin{array}{c}61,5 \\
\pm 14,1 \\
46,6 \\
\pm 9,1 \\
40,3 \\
\pm 18,6\end{array}$ & $\begin{array}{c}37,6 \\
\pm 14,1 \\
18,9 \\
\pm 4,6 \\
13,8 \\
\pm 3,6\end{array}$ & $\begin{array}{l}76,7 \\
\pm 13,5 \\
\\
54,2 \\
\pm 8,2\end{array}$ & $\begin{array}{l}35,9 \\
\pm 10,9\end{array}$ & $\begin{array}{c}61,8 \\
\pm 6,5 \\
61,5 \\
\pm 14,1 \\
64,7 \\
\pm 6,5\end{array}$ & $\begin{array}{c}24,7 \\
\pm 2,4 \\
12,9 \\
\pm 6,4 \\
13,6 \\
\pm 2,2\end{array}$ \\
\hline 7 & $\begin{array}{r}600 \\
3300\end{array}$ & $\begin{array}{c}53,5 \\
\pm 5,3 \\
60,5 \\
\pm 10 \\
72,0 \\
\pm 12,8\end{array}$ & $\begin{array}{c}20,4 \\
\pm 2,8 \\
15,0 \\
\pm 2,4 \\
15,1 \\
\pm 3,1\end{array}$ & $\begin{array}{l}74,0 \\
\pm 6,9 \\
60,1 \\
\pm 12,9 \\
60,5 \\
\pm 10,0\end{array}$ & $\begin{array}{c}25,6 \\
\pm 3,4 \\
13,2 \\
\pm 1,8 \\
12,2 \\
\pm 1,9\end{array}$ & $\begin{array}{c}53,2 \\
\pm 6,0 \\
53,5 \\
\pm 5,3 \\
74,0 \\
\pm 6,9\end{array}$ & $\begin{array}{c}15,8 \\
\pm 4,5 \\
11,1 \\
\pm 1,7 \\
12,1 \\
\pm 2,1\end{array}$ & $\begin{array}{c}64,7 \\
\pm 6,5 \\
61,8 \\
\pm 6,5 \\
54,7 \\
\pm 10,3\end{array}$ & $\begin{array}{c}41,4 \\
\pm 5,6 \\
23,7 \\
\pm 5,6 \\
15,2 \\
\pm 2,8\end{array}$ & $\begin{array}{c}61,5 \\
\pm 14,1 \\
64,0 \\
\pm 12,4 \\
61,8 \\
\pm 6,5\end{array}$ & $\begin{array}{c}31,6 \\
\pm 10,8 \\
23,2 \\
\pm 4,9 \\
20 \\
\pm 4,0\end{array}$ & $\begin{array}{c}40,3 \\
\pm 18,6 \\
46,6 \\
\pm 9,1 \\
76,7 \\
\pm 13,5\end{array}$ & $\begin{array}{c}14,7 \\
\pm 2,9 \\
12,4 \\
\pm 1,7 \\
16,4 \\
\pm 6,5\end{array}$ \\
\hline
\end{tabular}


Tabelle 33

Mittelwerte und Streunngen des organischen Stickstoffs [mg org. N/1]

\begin{tabular}{|c|c|c|c|c|c|c|c|c|c|c|c|c|c|}
\hline \multirow{2}{*}{$\begin{array}{l}\mathrm{O}_{2}-\text { Konz. } \\
\mathrm{BB} \\
\mathrm{mg} / \mathrm{l}\end{array}$} & \multirow{2}{*}{$\begin{array}{l}\text { Schlamm- } \\
\text { konz. BB } \\
\mathrm{mg} / \mathrm{l}\end{array}$} & \multicolumn{6}{|c|}{$\begin{array}{l}\text { Mittlere Temperatur BB }=>13^{\circ} \mathrm{C} \\
\text { Hydraulische Raumbelastung } \mathrm{m}^{3} / \mathrm{m}^{3} \cdot \text { Tag }\end{array}$} & \multicolumn{6}{|c|}{$\begin{array}{l}\text { Mittlere Temperatur BB }=<11^{\circ} \mathrm{C} \\
\text { Hydraulische Raumbelastung } \mathrm{m}^{3} / \mathrm{m}^{3} \cdot \text { Tag }\end{array}$} \\
\hline & & $\begin{array}{l}58 \\
Z\end{array}$ & $\mathrm{~A}$ & $\begin{array}{l}29 \\
Z\end{array}$ & A & $\begin{array}{l}9 \\
Z\end{array}$ & A & $\begin{array}{l}58 \\
Z\end{array}$ & A & 29 & A & $\begin{array}{l}9 \\
Z\end{array}$ & A \\
\hline 1 & $\begin{array}{r}600 \\
3300 \\
6000\end{array}$ & $\begin{array}{c}8,5 \\
\pm 1,7 \\
8,2 \\
\pm 1,1 \\
10,1 \\
\pm 1,5\end{array}$ & $\begin{array}{l}4,9 \\
\pm 0,4 \\
2,6 \\
\pm 0,4 \\
2,7 \\
\pm 0,5\end{array}$ & $\begin{array}{c}8,2 \\
\pm 1,1 \\
10,1 \\
\pm 1,2 \\
8,6 \\
\pm 1,2\end{array}$ & $\begin{array}{c}3,7 \\
\pm 0,6 \\
2,9 \\
\pm 0,5 \\
2,5 \\
\pm 0,5\end{array}$ & $\begin{array}{c}8,3 \\
\pm 1,4 \\
8,9 \\
\pm 0,4 \\
10,1 \\
\pm 1,2\end{array}$ & $\begin{array}{c}3,7 \\
\pm 0,9 \\
1,3 \\
\pm 0,2 \\
1,3 \\
\pm 0,3\end{array}$ & $\begin{array}{c}10,4 \\
\pm 1,9 \\
11,3 \\
\pm 3,3 \\
10,2 \\
\pm 3,3\end{array}$ & $\begin{array}{c}5,5 \\
\pm 0,6 \\
4,4 \\
\pm 1,0 \\
4,2 \\
\pm 1,2\end{array}$ & $\begin{array}{c}9,7 \\
\pm 1,6 \\
10,3 \\
\pm 1,6 \\
10,6 \\
\pm 1,5\end{array}$ & $\begin{array}{l}5,3 \\
\pm 1,2 \\
3,3 \\
\pm 0,7 \\
3,1 \\
\pm 1,2\end{array}$ & $\begin{array}{c}10,4 \\
\pm 1,9 \\
9,7 \\
\pm 1,6 \\
11,3 \\
\pm 3,3\end{array}$ & $\begin{array}{l}4,0 \\
\pm 0,3 \\
2,4 \\
\pm 1,3 \\
1,3 \\
\pm 0,4\end{array}$ \\
\hline 4 & $\begin{array}{r}600 \\
3300\end{array}$ & $\begin{array}{c}10,6 \\
\pm 0,7 \\
8,6 \\
\pm 1,2 \\
10,1 \\
\pm 1,2\end{array}$ & $\begin{array}{c}4,2 \\
\pm 0,4 \\
3,0 \\
\pm 0,3 \\
2,9 \\
\pm 0,3\end{array}$ & $\begin{array}{c}10,6 \\
\pm 0,7 \\
8,9 \\
\pm 0,4 \\
7,9 \\
\pm 0,8\end{array}$ & $\begin{array}{l}3,8 \\
\pm 0,6 \\
2,2 \\
\pm 0,5 \\
1,6 \\
\pm 0,9\end{array}$ & $\begin{array}{c}10,2 \\
\pm 1,5 \\
10,1 \\
\pm 1,5 \\
10,6 \\
\pm 0,7\end{array}$ & $\begin{array}{c}3,6 \\
\pm 0,5 \\
2,5 \\
\pm 1,6 \\
1,5 \\
\pm 0,8\end{array}$ & $\begin{array}{c}13,2 \\
\pm 1,7 \\
10,3 \\
\pm 1,6 \\
9,3 \\
\pm 1,8\end{array}$ & $\begin{array}{c}7,8 \\
\pm 1,9 \\
4,7 \\
\pm 3,5 \\
3,1 \\
\pm 1,4\end{array}$ & $\begin{array}{c}10,1 \\
\pm 3,3\end{array}$ & $\begin{array}{l}6,4 \\
\pm 1,3\end{array}$ & $\begin{array}{c}11,5 \\
\pm 1,1 \\
13,2 \\
\pm 1,7 \\
9,0 \\
\pm 1,0\end{array}$ & $\begin{array}{c}4,9 \\
\pm 0,5 \\
3,2 \\
\pm 1,4 \\
1,2 \\
\pm 0,3\end{array}$ \\
\hline 7 & 6000 & $\begin{array}{c}7,9 \\
\pm 0,8 \\
8,5 \\
\pm 2,2 \\
10,4 \\
\pm 4,2\end{array}$ & $\begin{array}{l}4,1 \\
\pm 0,07 \\
1,8 \\
\pm 0,8 \\
2,1 \\
\pm 0,9\end{array}$ & $\begin{array}{c}9,5 \\
\pm 0,9 \\
10,1 \\
\pm 1,5 \\
8,5 \\
\pm 2,2\end{array}$ & $\begin{array}{c}4,9 \\
\pm 1,1 \\
3,4 \\
\pm 2,4 \\
1,2 \\
\pm 0,5\end{array}$ & $\begin{array}{l}8,6 \\
\pm 1,2 \\
7,9 \\
\pm 0,8 \\
9,5 \\
\pm 0,9\end{array}$ & $\begin{array}{c}2,6 \\
\pm 0,4 \\
1,5 \\
\pm 0,4 \\
1,5 \\
\pm 0,7\end{array}$ & $\begin{array}{c}9,0 \\
\pm 1,0 \\
11,5 \\
\pm 1,1 \\
9,7 \\
\pm 1,6\end{array}$ & $\begin{array}{l}6,9 \\
\pm 1,8 \\
4,1 \\
\pm 0,7 \\
2,8 \\
\pm 0,3\end{array}$ & $\begin{array}{c}13,2 \\
\pm 1,7 \\
10,4 \\
\pm 1,9 \\
11,5 \\
\pm 1,1\end{array}$ & $\begin{array}{l}6,6 \\
\pm 2,2 \\
3,9 \\
\pm 0,5 \\
2,9 \\
\pm 0,4\end{array}$ & $\begin{array}{c}9,3 \\
+1,8 \\
10,4 \\
\pm 1,6 \\
10,2 \\
\pm 3,3\end{array}$ & $\begin{array}{c}3,5 \\
\pm 1,3 \\
2,7 \\
\pm 0,6 \\
2,6 \\
\pm 1,0\end{array}$ \\
\hline
\end{tabular}


Tabelle 34

Mittelwerte und Streuungen des $B S B_{5} \quad[\mathrm{mg} \mathrm{O} / 1]$

\begin{tabular}{|c|c|c|c|c|c|c|c|c|c|c|c|c|c|}
\hline \multirow{2}{*}{$\begin{array}{l}\mathrm{O}_{2} \text {-Konz. } \\
\mathrm{BB} \\
\mathrm{mg} / 1\end{array}$} & \multirow{2}{*}{$\begin{array}{l}\text { Schlamm- } \\
\text { konz. BB } \\
\mathrm{mg} / 1\end{array}$} & \multicolumn{6}{|c|}{$\begin{array}{l}\text { Mittlere Temperatur } \mathrm{BB}=>13^{\circ} \mathrm{C} \\
\text { Hydraulische Raumbelastung } \mathrm{m}^{3} / \mathrm{m}^{3} \cdot \text { Tag }\end{array}$} & \multicolumn{6}{|c|}{$\begin{array}{l}\text { Mittlere Temperatur } \mathrm{BB}=<11^{\circ} \mathrm{C} \\
\text { Hydraulische Raumbelastung } \mathrm{m}^{3} / \mathrm{m}^{3} \cdot \text { Tag }\end{array}$} \\
\hline & & $\begin{array}{l}58 \\
Z\end{array}$ & A & $\begin{array}{l}29 \\
Z\end{array}$ & $\mathrm{~A}$ & $\begin{array}{l}9 \\
Z\end{array}$ & A & $\begin{array}{l}58 \\
Z\end{array}$ & $\mathrm{~A}$ & $\begin{array}{l}29 \\
Z\end{array}$ & $\mathrm{~A}$ & $\begin{array}{l}9 \\
Z\end{array}$ & $\mathrm{~A}$ \\
\hline 1 & 6000 & $\begin{array}{c}105 \\
\pm 23 \\
95 \\
\pm 13 \\
122 \\
\pm 21\end{array}$ & $\begin{array}{l}46 \\
\pm 10 \\
12 \\
\pm 3 \\
18 \\
\pm 5\end{array}$ & $\begin{array}{l}95 \\
\pm 13 \\
94 \\
\pm 18 \\
97 \\
\pm 11\end{array}$ & $\begin{array}{c}20 \\
\pm 4 \\
10 \\
\pm 3 \\
16 \\
\pm 3\end{array}$ & $\begin{array}{c}91 \\
\pm 15 \\
149 \\
\pm 25 \\
94 \\
\pm 18\end{array}$ & $\begin{array}{c}18 \\
\pm 5 \\
9 \\
\pm 3 \\
9 \\
\pm 5\end{array}$ & $\begin{array}{c}110 \\
\pm 22 \\
91 \\
\pm 34 \\
129 \\
\pm 26\end{array}$ & $\begin{array}{c}65 \\
\pm 8 \\
17 \\
\pm 7 \\
23 \\
\pm 5\end{array}$ & $\begin{array}{c}105 \\
\pm 26 \\
113 \\
\pm 58 \\
135 \\
\pm 15\end{array}$ & $\begin{array}{c}52 \\
\pm 19 \\
18 \\
\pm 6 \\
11 \\
\pm 2\end{array}$ & $\begin{array}{c}110 \\
\pm 22 \\
105 \\
\pm 26 \\
91 \\
\pm 34\end{array}$ & $\begin{array}{l}19 \\
\pm 6 \\
8 \\
\pm 2 \\
7 \\
\pm 5\end{array}$ \\
\hline 4 & 6000 & $\begin{array}{c}116 \\
\pm 21 \\
97 \\
\pm 11 \\
94 \\
\pm 18\end{array}$ & $\begin{array}{c}28 \\
\pm 5 \\
19 \\
\pm 5 \\
12 \\
\pm 4\end{array}$ & $\begin{array}{c}116 \\
\pm 21 \\
149 \\
\pm 25 \\
138 \\
\pm 31\end{array}$ & $\begin{array}{c}20 \\
\pm 3 \\
11 \\
\pm 2 \\
15 \\
\pm 7\end{array}$ & $\begin{array}{c}118 \\
\pm 25 \\
122 \\
\pm 21 \\
116 \\
\pm 21\end{array}$ & $\begin{array}{c}11 \\
\pm 2 \\
11 \\
\pm 5 \\
8 \\
\pm 6\end{array}$ & $\begin{array}{c}115 \\
\pm 20 \\
113 \\
\pm 58 \\
115 \\
\pm 40\end{array}$ & $\begin{array}{l}55 \\
\pm 13 \\
16 \\
\pm 9 \\
15 \\
\pm 4\end{array}$ & $\begin{array}{l}135 \\
\pm 15\end{array}$ & $\begin{array}{l}55 \\
\pm 28\end{array}$ & $\begin{array}{c}117 \\
\pm 12 \\
115 \\
\pm 20 \\
119 \\
\pm 21\end{array}$ & $\begin{array}{l}27 \\
\pm 8 \\
7 \\
\pm 2 \\
6 \\
\pm 1\end{array}$ \\
\hline 7 & 6000 & $\begin{array}{c}138 \\
\pm 31 \\
101 \\
\pm 17 \\
92 \\
\pm 15\end{array}$ & $\begin{array}{c}37 \\
\pm 6 \\
9 \\
\pm 1 \\
15 \\
\pm 9\end{array}$ & $\begin{array}{c}143 \\
\pm 34 \\
122 \\
\pm 21 \\
101 \\
\pm 17\end{array}$ & $\begin{array}{c}34 \\
\pm 8 \\
14 \\
\pm 3 \\
7 \\
\pm 2\end{array}$ & $\begin{array}{c}97 \\
\pm 11 \\
138 \\
\pm 31 \\
143 \\
\pm 34\end{array}$ & $\begin{array}{c}13 \\
\pm 2 \\
16 \\
\pm 8 \\
14 \\
\pm 3\end{array}$ & $\begin{array}{c}119 \\
\pm 21 \\
117 \\
\pm 12 \\
105 \\
\pm 26\end{array}$ & $\begin{array}{l}50 \\
\pm 12 \\
24 \\
\pm 10 \\
17 \\
\pm 6\end{array}$ & $\begin{array}{c}115 \\
\pm 20 \\
110 \\
\pm 22 \\
117 \\
\pm 12\end{array}$ & $\begin{array}{c}42 \\
\pm 22 \\
18 \\
\pm 1,7 \\
23 \\
\pm 7\end{array}$ & $\begin{array}{c}115 \\
\pm 40 \\
113 \\
\pm 58 \\
129 \\
\pm 26\end{array}$ & $\begin{array}{c}22 \\
\pm 11 \\
9 \\
\pm 2 \\
17 \\
\pm 7\end{array}$ \\
\hline
\end{tabular}




\section{Liste der Bezeichnungen}

$R_{h}$ hydraulische Raumbelastung $\left[\mathrm{m}^{3} \mathrm{Abwasser} / \mathrm{m}^{3}\right.$ Belüftungsbecken $\left.\cdot \mathrm{Tag}\right]$

$\mathrm{O}$ Sauerstoffkonzentration im Belüftungsbecken $\left[\mathrm{g} \mathrm{O} / \mathrm{m}^{3}\right.$ Belebtschlamm]

$t \quad$ Zeit $[\mathrm{h}$, Tage $]$

* Organismenkonzentration allgemein $\left[\mathrm{g} / \mathrm{m}^{3}\right]$

$x_{1}$ Organismenkonzentration in einem Fermenter $\left[\mathrm{g} / \mathrm{m}^{3}\right]$

$x_{1}^{\prime}$ Schlammkonzentration im Belüftungsbecken (Organismen + primäre Schwebestoffe) $\left[\mathrm{g} / \mathrm{m}^{3}\right]$

$s_{R} \quad$ Substratkonzentration im Zufluss zum Belüftungsbecken $\left[\mathrm{g} / \mathrm{m}^{3}\right]$

$s_{R}^{*}$ Konzentration der abbaubaren Stoffe im Zufluss zum Belüftungsbecken $\left[\mathrm{g} / \mathrm{m}^{3}\right]$ Konzentration der abbaubaren Stoffe im Abfluss des Belïftungsbeckens $\left[\mathrm{g} / \mathrm{m}^{3}\right]$

$s$ Substratkonzentration im Abfluss des Belüftungs-, bzw. Nachklärbeckens $\left[\mathrm{g} / \mathrm{m}^{3}\right]$

$s_{i} \quad$ Konzentration der gelösten nicht angreifbaren Verbindungen im Abfluss des Belüftungsbeckens $\left[\mathrm{g} / \mathrm{m}^{3}\right]$

$K_{s}$ Substratkonzentration für halbe Wachstumsgeschwindigkeit der Organismen $\left[\mathrm{g} / \mathrm{m}^{3}\right]$

$\mu \quad$ Wachstumsgeschwindigkeit der Organismen $\left[\mathrm{h}^{-1}\right]$

$\mu_{m}$ maximale Wachstumsgeschwindigkeit der Organismen bei unbegrenzter Substratzufuhr $\left[\mathrm{h}^{-1}\right]$

$x_{2}$ abfliessende Organismenmenge aus einem Fermentationssystem im Beharrungszustand (im gesamten Flüssigkeitsdurchfluss suspendiert) $\left[\mathrm{g} / \mathrm{m}^{3}\right]$

$x_{2}^{\prime}$ Uberschußschlamm aus einer Belebtschlammanlage, gemessen als Konzentration der durchfliessenden Abwassermenge $\left[\mathrm{g} / \mathrm{m}^{3}\right.$ Abwasser]

a Rücklaufschlammrate [ $\mathrm{m}^{3}$ Zufluss/m $\mathrm{m}^{3}$ Rücklaufschlamm]

$f$ Anlagenzufluss allgemein $\left[\mathrm{m}^{3} /\right.$ Zeiteinheit $]$

C Konzentrierungseffekt im Nachklärbecken, Organismenkonzentration Zufluss/Org. konz. Rücklaufschlamm

$Y$ Ausnützungskoeffizient für Substrat in einem Fermenter, $g$ Organismenzuwachs/g verarbeitetes Substrat

$Y_{S}^{\prime}$ Bruttoausnützungskoeffizient in einem Belebtschlammsystem [g org. $\mathrm{C}$ im Uberschußschlamm/g org. C eliminiert]

$l_{R} \quad$ gelöste Schmutzstoffe im Belüftungsbeckenzufluss $\left[\mathrm{g} / \mathrm{m}^{3}\right]$

$i_{R} \quad$ ungelöste Schmutzstoffe im Belüftungsbeckenzu fluss $\left[\mathrm{g} / \mathrm{m}^{3}\right]$

$i_{s}$ im Belüftungsbecken biologisch nicht angegriffene, ungelöste Schmutzstoffe des Belüftungsbeckenzuflusses $\left[\mathrm{g} / \mathrm{m}^{3}\right]$

$N_{x}$ Stickstoffgehalt eines reinen Bakterienbelebtschlamms, \% $\mathrm{N}$

$N_{x}$, Stickstoffgehalt eines Belebtschlamms, \% $N$

$S_{b} \quad$ Schlammbelastung allgemein [kg Substrat $/ \mathrm{kg}$ Trockensubstanz $\cdot$ Tag]

$\eta_{B} \quad$ Reinigungseffekt gemessen am $\mathrm{BSB}_{5}, \%$

$\eta_{C} \quad$ Reinigungseffekt, gemessen am org. $\mathrm{C}, \%$

$\eta_{N}$ Reinigungseffekt, gemessen am org. $\mathrm{N}, \%$

$t^{\circ}$ Temperatur ${ }^{\circ} \mathrm{C}$

$\varepsilon \quad$ Eliminationsgeschwindigkeit [g Substratelimination/g Organismen $\cdot \mathrm{h}$ ]

\section{LITER A TURVERZEICHNIS}

[I] BROWNLEE, K. A., Statistical theory and metbodology in science and engineering (J. Wiley \& Sons, Inc., New York, London I960).

[2] Downing, A.L., Painter, H.A., und Knowles, G., Nitrification in the activated sludge process, J. Proc. Inst. Sew. Purif., 2-25 (I963).

[3] EMDE, W., VON DER, Beitrag zu Versucben zur Abwasserreinigung mit belebtem Scblamm. Veröff. Inst. f. Siedlungswasserwirtschaft, TH Hannover, H. 1 (1957). 
[4] FaIR, G. M., und Thomas, H.A., Concept of interface and loading in submerged aerobic biological semage treatment systems, J. Proc. Inst. Sew. Purif., $235-248$ (1950).

[s] Herbert, D., A theoretical analysis of continuous culture systems, Continuous culture of microorganisms. S.C.I. Monogr. No. I2, Soc. Chem. Ind. London, 21-53 (196I).

[6] Herbert, D., Elsworth, R., und Telling, R.C., The continuous culture of bacteria; a tbeoretical and experimental study, J. gen. Microbiol. 14, 60I-622 (1956).

[7] HunkeN, K.H., Untersuchungen über den Reinigungsperlauf und den Sauerstoffperbrauch bei der Abpasserreinigung durcb das Belebtschlammperfabren. Stuttgarter Ber. z. Siedlungswasserwirtschaft, Nr. 4 (Verlag R. Oldenbourg, München 1960).

[8] IMHOFF, KL., Zur Berecbnung pon Belebungsbecken, GWF 104, I494-I498 (1963).

[9] Monod, J., Recherches sur la croissance des cultures bactériennes (Edit. Hermann, Paris 1942).

[Io] Novick, A., und SZILlaRd, L., Experiments with the chemostat on spontaneous mutations of bacteria, Proc. Nat. Acad, Sci., Wash. 36, 708 (I950).

[i I] SAWYER, C.N., Frame, J.D., und WoOD, J.P., Revised concepts on biological treatment, Sew. Ind. Wastes 27, 929-940 (I955).

[12] SCHULzE, K.L., und LIPE, R.S., Relationship between substrate concentration, gromtb rate and respiration rate of Escherichia coli in continuous culture, Arch. Mikrobiol. 48, I-20 (I964).

[I3] SLYKE, D. D. VAN, und FolCh, J., Manometric carbon determination, J. Biol. Chem. 136, 509-54I (I940).

[14] Teissier, G., Les lois quantitatipes de la croissance, Ann. Physiol. physicochim. biol. 12, 527-586 (1936).

[I5] VIEHL, K., Der Einfluss des Sauerstoffgebaltes auf den biochemischen Sauerstoffbedarf und auf die natürlicbe Selbstreinigung des Wassers somie auf die Wirkungsmeise des Belebtscblamms, Z. Hyg. Inf.krankh. 122, 533-549 (1940).

[16] VIEHL, K., und MEISSNER, B., Über den Einfluss der Temperatur und der Fabreszeit auf die biologiscbe Abwasserreinigung", Z. Bakt. II, 91, 14-39 (1934).

[17] WUHRMANN, K., Die beutigen Einrichtungen für verfabrenstecbnische Forscbungen über biologiscbe Abmasserreinigung und für Studien an Fliessgemässern in der Versucbsstation Tüffenmies der EAWAG, Verb. Schweiz. Abwasserfachleute, Verb.-Ber. Nr. 44/2 (I957).

[1 8] WUHRMANN, K., Über die Sumerstoff persorgung flockiger oder zoogloenartiger Aggregationen aerober Mikroorganismen, Z. allg. Path. u. Bakteriol. 20, 567-576 (1957).

[19] WUHRMANN, K., Effect of oxygen tension on biocbemical reactions in semage purification processes, in: Advances in Biological Waste Treatment. Proc. Third Conf. on Biol. Waste Treatment, Manhattan College I960 (Edit. W. W. Eckenfelder und J. McCabe, Pergamon Press, Oxford, p. 27-40, 1963).

[20] WuhrmanN, K., Die Grundlagen der Dimensionierung der Belïftung bei Belebtscblammanlagen, Schweiz, Z. Hydrol. 26, Fasc. 2 (1964).

[2I] WuHRmann, K., und BEUST, F. von, Zur Theorie des Belebtscblammperfubrens, II. Uber den Mechanismus der Elimination gelöster organiscber Stoffe aus Abwasser bei der biologiscben Reinigung. Schweiz. Z. Hydrol. 20, 3 I I-330 (1958).

Separatdruck aus der Schweiz. Zeitschrift für Hydrologie 26, Fasc. 2 (1964) Herausgegeben mit Unterstützung der Stiftung der Wirtschaft zur Förderung des Gewässerschutzes in der Schweiz 